\title{
Coulomb scattering in the massless Nelson model III. Ground state wave functions and non-commutative recurrence relations
}

\author{
Wojciech Dybalski \\ Zentrum Mathematik, Technische Universität München, \\ and \\ Fakultät für Mathematik, Ludwig-Maximilians-Universität München \\ E-mail: dybalski@ma.tum.de \\ Alessandro Pizzo \\ Dipartimento di Matematica, Università di Roma "Tor Vergata" \\ E-mail: pizzo@axp.mat.uniroma2.it
}

\begin{abstract}
Let $H_{P, \sigma}$ be the single-electron fiber Hamiltonians of the massless Nelson model at total momentum $P$ and infrared cut-off $\sigma>0$. We establish detailed regularity properties of the corresponding $n$-particle ground state wave functions $f_{P, \sigma}^{n}$ as functions of $P$ and $\sigma$. In particular, we show that

$$
\left|\partial_{P^{j}} f_{P, \sigma}^{n}\left(k_{1}, \ldots, k_{n}\right)\right|,\left|\partial_{P^{j}} \partial_{P^{j^{\prime}}} f_{P, \sigma}^{n}\left(k_{1}, \ldots, k_{n}\right)\right| \leq \frac{1}{\sqrt{n !}} \frac{\left(c \lambda_{0}\right)^{n}}{\sigma^{\delta_{\lambda_{0}}}} \prod_{i=1}^{n} \frac{\chi_{[\sigma, \kappa)}\left(k_{i}\right)}{\left|k_{i}\right|^{3 / 2}},
$$

where $c$ is a numerical constant, $\lambda_{0} \mapsto \delta_{\lambda_{0}}$ is a positive function of the maximal admissible coupling constant which satisfies $\lim _{\lambda_{0} \rightarrow 0} \delta_{\lambda_{0}}=0$ and $\chi_{[\sigma, \kappa)}$ is the (approximate) characteristic function of the energy region between the infrared cut-off $\sigma$ and the ultraviolet cut-off $\kappa$. While the analysis of the first derivative is relatively straightforward, the second derivative requires a new strategy. By solving a non-commutative recurrence relation we derive a novel formula for $f_{P, \sigma}^{n}$ with improved infrared properties. In this representation $\partial_{P^{j}} \partial_{P^{j}} f_{P, \sigma}^{n}$ is amenable to sharp estimates obtained by iterative analytic perturbation theory in part II of this series of papers. The bounds stated above are instrumental for scattering theory of two electrons in the Nelson model, as explained in part I of this series.
\end{abstract}

\section{Introduction}

Recently there has been some revival of interest in infrared problems within the theoretical physics community, triggered by the work of Strominger et al. on soft photon theorems (see [St17] for a review). Apart from emphasizing the richness of infrared physics, this reference states clearly that 
the absence of an infrared regular $S$-matrix in QED 'is a big elephant in the room of mathematical quantum field theory'. And indeed, the infrared problem has been a subject of continuing research in the mathematical physics community for many decades. Also the present paper, which advances our long term investigation of two-electron scattering in a simplified model of QED, stems from this tradition and provides evidence for the richness of infrared mathematics. Namely, we find an unexpected connection between infrared problems and the theory of non-commutative recurrence relations. This theory turns out to be a robust method to demonstrate infrared regularity of physical quantities which suffer from superficial infrared divergencies even after implementation of multiscale techniques.

To summarize briefly the theory of non-commutative recurrence relations, we consider two, possibly non-commuting, linear operators $\hat{a}$ and $\hat{b}$ on a vector space $X$. Suppose the following relation holds

$$
x_{n}=\hat{a} x_{n-1}+\hat{b} x_{n-2} \text {, }
$$

for a sequence of vectors $\left\{x_{n}\right\}_{n \in \mathbb{N}_{0}}$ from $X$ with given initial conditions $x_{0}$ and $x_{1}=\hat{a} x_{0}$. The first closed-form solution of such a non-commutative recurrence was given in [JMNP07, JNM08] and then applied to a computation of scattering amplitudes in perturbative QFT in [Pu15]. It was shown by induction in these references that it has the form (cf. formula (2.7) of [Pu15])

$$
x_{n}=\sum_{i_{1}+2 i_{2}=n}\left\{\hat{a}^{\left(i_{1}\right)}, \hat{b}^{\left(i_{2}\right)}\right\} x_{0}
$$

where the bracket $\left\{\hat{a}^{\left(i_{1}\right)}, \hat{b}^{\left(i_{2}\right)}\right\}$ denotes the sum over all possible distinct permutations of factors $\hat{a}$, $\hat{b}$ each one appearing $i_{1}, i_{2}$ times, respectively. In our work we establish a different representation for the solution of (1.1) which is more suitable for our applications. It has the form

$$
x_{n}=\operatorname{Op}_{\hat{a}, \hat{b}}^{(n)}\left[\exp \left(\sum_{i=1}^{n-1} b_{i+1, i} \partial_{a_{i+1}} \partial_{a_{i}}\right) a_{n} a_{n-1} \ldots a_{1}\right] x_{0},
$$

where $a_{i}, b_{i+1, i}$ are real variables so that $a_{n} a_{n-1} \ldots a_{1}$ is a monomial on which $b_{i+1, i} \partial_{a_{i+1}} \partial_{a_{i}}$ acts by replacing $a_{i+1} a_{i}$ with $b_{i+1, i}$. The 'quantization map' $\mathrm{Op}_{\hat{a}, \hat{b}}^{(n)}$ replaces every $a_{i^{\prime}}$ in the resulting polynomial with the operator $\hat{a}$ and every $b_{i^{\prime}+1, i^{\prime}}$ with the operator $\hat{b}$ while keeping the order specified by the indices. For a more detailed discussion and a very simple proof that (1.3) solves (1.1) we refer to Subsection 3.1.

Let us now proceed to applications of the theory of non-commutative recurrence relations to infrared problems. Let $H_{P, \sigma}$ be the fiber Hamiltonian of the Nelson model at total momentum $P$ with the infrared cut-off $\sigma>0$. It is a self-adjoint operator on the symmetric Fock space over $L^{2}\left(\mathbb{R}^{3}\right)$ given by

$$
H_{P, \sigma}:=\frac{1}{2}\left(P-P_{\mathrm{f}}\right)^{2}+H_{\mathrm{f}}+\int d^{3} k v_{\bar{\alpha}}^{\sigma}(k)\left(b(k)+b^{*}(k)\right)
$$

where $b^{*}(k), b(k)$ are the (improper) creation and annihilation operators, $P_{\mathrm{f}}:=\int d^{3} k k b^{*}(k) b(k)$ is the photon momentum and the form-factor $v_{\bar{\alpha}}^{\sigma}(k):=\lambda \chi_{[\sigma, k)}(k)|k|^{\bar{\alpha}} / \sqrt{2|k|}$ is defined precisely by formula (2.2) below. It contains the coupling constant $\lambda$ whose absolute value will be kept 
sufficiently small in our discussion and the regularity parameter $\bar{\alpha} \geq 0$, which can be set to zero for the purpose of the present paper. (We keep it here for consistency with [DP12] where $\bar{\alpha}>0$ was assumed). This Hamiltonian has a normalized ground state vector $\breve{\psi}_{P, \sigma}$, with the phase fixed in (2.4), corresponding to the simple eigenvalue $E_{P, \sigma}$. In [DP17] we derived the following formulas for the first and second derivative of $\breve{\psi}_{P, \sigma}$ w.r.t. to $P$

$$
\begin{aligned}
& \partial_{P j} \check{\psi}_{P, \sigma}=R_{P, \sigma}\left(\Lambda_{P, \sigma}\right)^{j} \check{\psi}_{P, \sigma}, \\
& \partial_{P^{j}} \partial_{P^{j^{\prime}}} \breve{\psi}_{P, \sigma}=\left(\bar{Q}_{P, \sigma}^{\perp} R_{P, \sigma}\left(\Lambda_{P, \sigma}\right)^{j^{\prime}} R_{P, \sigma}\left(\Lambda_{P, \sigma}\right)^{j} \check{\psi}_{P, \sigma}+\left(j \leftrightarrow j^{\prime}\right)\right) \\
& -\breve{\psi}_{P, \sigma}\left\langle\check{\psi}_{P, \sigma},\left(\Lambda_{P, \sigma}\right)^{j^{\prime}} R_{P, \sigma}^{2}\left(\Lambda_{P, \sigma}\right)^{j} \breve{\psi}_{P, \sigma}\right\rangle \text {, } \\
& R_{P, \sigma}:=\left(H_{P, \sigma}-E_{P, \sigma}\right)^{-1}, \quad \Lambda_{P, \sigma}:=\nabla E_{P, \sigma}-\left(P-P_{\mathrm{f}}\right), \quad \bar{Q}_{P, \sigma}:=\left|\breve{\psi}_{P, \sigma}\right\rangle\left\langle\breve{\psi}_{P, \sigma}\right| .
\end{aligned}
$$

We recall that $\Lambda_{P, \sigma}$ has the property $\left\langle\breve{\psi}_{P, \sigma}, \Lambda_{P, \sigma} \breve{\psi}_{P, \sigma}\right\rangle=0$, so the above expressions are well defined. The control of the behaviour of $\partial_{P^{j}} \breve{\psi}_{P, \sigma}, \partial_{P^{j}} \partial_{P^{\prime}} \breve{\psi}_{P, \sigma}$ as $\sigma \rightarrow 0$ is a difficult problem in spectral theory, concerning eigenvalues at the bottom of the continuous spectrum. However, using iterative analytic perturbation theory we established the following estimates in [DP17]

$$
\begin{aligned}
& \left\|R_{P, \sigma}\left(\Lambda_{P, \sigma}\right)^{j} \check{\psi}_{P, \sigma}\right\| \leq \frac{c}{\sigma^{\delta_{\lambda_{0}}}}, \\
& \left\|\bar{Q}_{P, \sigma}^{\perp} R_{P, \sigma}\left(\Lambda_{P, \sigma}\right)^{j} R_{P, \sigma}\left(\Lambda_{P, \sigma}\right)^{j^{\prime}} \check{\psi}_{P, \sigma}\right\| \leq \frac{c}{\sigma^{\delta_{\lambda_{0}}}},
\end{aligned}
$$

where $c$ is a universal constant and $\lambda_{0} \mapsto \delta_{\lambda_{0}}$ is positive and satisfies $\lim _{\lambda_{0} \rightarrow 0} \delta_{\lambda_{0}}=0$. Thus the infrared singularity of this estimate is very mild in the weak coupling regime. Obviously, from (1.5)-(1.9) we obtain

$$
\left\|\partial_{P^{j}} \breve{\psi}_{P, \sigma}\right\|, \quad\left\|\partial_{P^{j}} \partial_{P^{\prime}} \breve{\psi}_{P, \sigma}\right\| \leq \frac{c}{\sigma^{\delta_{\lambda_{0}}}} .
$$

The purpose of the present paper is to obtain bounds similar to (1.10) also for the $n$-photon wave functions $\left\{f_{P, \sigma}^{n}\right\}_{n \in \mathbb{N}_{0}}$ of $\breve{\psi}_{P, \sigma}$ using the spectral ingredients (1.8), (1.9) and their slight generalizations stated in Theorem 6.2 below. Our main results, stated precisely in Theorem 2.2 below, include the following estimates

$$
\left|\partial_{P^{j}} f_{P, \sigma}^{n}\left(k_{1}, \ldots, k_{n}\right)\right|, \quad\left|\partial_{P^{j}} \partial_{P^{j^{\prime}}} f_{P, \sigma}^{n}\left(k_{1}, \ldots, k_{n}\right)\right| \leq \frac{1}{\sqrt{n !}} \frac{c^{n}}{\sigma^{\delta_{\lambda_{0}}}} \prod_{i=1}^{n} \frac{v_{\bar{\alpha}}^{\sigma}\left(k_{i}\right)}{\left|k_{i}\right|}, \quad n \geq 0 .
$$

As explained in detail in [DP12], these bounds constitute crucial technical inputs for the construction of two-electron scattering states. The momentum derivatives play a crucial role in [DP12] via non-stationary phase arguments. The control up to the second derivative is dictated by the Cook's method.

The ground state wave function is expressed by the following formula

$$
f_{P, \sigma}^{n}\left(k_{1}, \ldots, k_{n}\right):=\frac{1}{\sqrt{n !}}\left\langle\Omega, b\left(k_{1}\right) \ldots b\left(k_{n}\right) \check{\psi}_{P, \sigma}\right\rangle .
$$

We define $\check{f}_{P, \sigma}^{n}\left(k_{1}, \ldots, k_{n}\right):=b\left(k_{1}\right) \ldots b\left(k_{n}\right) \check{\psi}_{P, \sigma}$ and set $\underline{k}_{n}:=k_{1}+\cdots+k_{n},|\underline{k}|_{n}:=\left|k_{1}\right|+\cdots+\left|k_{n}\right|$. Making use of the fact that $H_{P, \sigma} \check{\psi}_{P, \sigma}=E_{P, \sigma} \breve{\psi}_{P, \sigma}$ and of the canonical commutation relations, one 
easily gets

$\check{f}_{P, \sigma}^{n}\left(k_{1}, \ldots, k_{n}\right)=(-) R_{P, \sigma ; n} \sum_{i=1}^{n} v_{\bar{\alpha}}^{\sigma}\left(k_{i}\right) \check{f}_{P, \sigma}^{n-1}\left(k_{1}, \ldots \check{i} \ldots, k_{n}\right), \quad R_{P, \sigma ; n}:=\left(H_{P-\underline{k}_{n}, \sigma}-E_{P, \sigma}+|\underline{k}|_{n}\right)^{-1}$,

with $\check{f}_{P, \sigma}^{0}=\check{\psi}_{P, \sigma}$. Suitably interpreted, this is a trivial example of the recurrence (1.1) with $\hat{b}=0$. The solution has the form

$$
\check{f}_{P, \sigma}^{n}=(-1)^{n} n ! P_{\mathrm{sym}}\left(v_{\bar{\alpha} ; 1}^{\sigma} \ldots v_{\bar{\alpha} ; n}^{\sigma}\right)\left(R_{P, \sigma ; n} \ldots R_{P, \sigma ; 1}\right) \check{\psi}_{P, \sigma},
$$

where $P_{\text {sym }}$ denotes symmetrization in $k_{1}, \ldots, k_{n}$ and $\left(v_{\bar{\alpha} ; 1}^{\sigma} \ldots v_{\bar{\alpha} ; n}^{\sigma}\right)\left(k_{1}, \ldots, k_{n}\right):=v_{\bar{\alpha}}^{\sigma}\left(k_{1}\right) \ldots v_{\bar{\alpha}}^{\sigma}\left(k_{n}\right)$. By substituting (1.14) to (1.12), we reproduce the standard formula for the wave functions due to Fröhlich [Fr73, Fr74].

The next task is to study the derivatives of (1.14) so as to obtain estimates (1.11). Here the rules of the game are dictated by the following relations:

$$
\partial_{P^{j}} \breve{\psi}_{P, \sigma}=R_{P, \sigma}\left(\Lambda_{P, \sigma}\right)^{j} \breve{\psi}_{P, \sigma}, \quad \partial_{P j} R_{P, \sigma ; i}=R_{P, \sigma ; i}\left(\Lambda_{P, \sigma}^{i}\right)^{j} R_{P, \sigma ; i}, \quad\left\|R_{P, \sigma ; i}\right\| \leq c|\underline{k}|_{i}^{-1}
$$

where $\Lambda_{P, \sigma}^{i}:=\Lambda_{P, \sigma}+\underline{k}_{i}$ and the first identity simply recalls (1.5). Thus the action of $\partial_{P^{j}}$ typically increases the number of resolvents. If the additional resolvent comes from the second identity in (1.15), we cannot apply the standard resolvent bound $\left\|R_{P, \sigma ; i}\right\| \leq c|\underline{k}|_{i}^{-1}$, as this would lead to more factors $\left|k_{i^{\prime}}\right|^{-1}$ than those appearing in (1.11). Furthermore, if we exploited the support property of $v_{\bar{\alpha}}^{\sigma}$ to write $\left|k_{i^{\prime}}\right|^{-1} \leq \sigma^{-1}$, this would destroy the mild infrared behaviour of our main result given by $\sigma^{-\delta_{\lambda_{0}}}$. Thus the additional resolvents must be absorbed by the spectral ingredients (1.8), (1.9). This is possible as long as the relevant expressions have the alternating structure of resolvents separated by $\Lambda_{P, \sigma}$ factors. As we demonstrate in Subsection A.2, this strategy works for $\partial_{P^{j}} f_{P, \sigma}^{n}$. However, the analysis of the second derivative based on (1.14) faces serious problems. In fact, already in the case of $n=1$ we encounter the following contribution

$$
\partial_{P^{j}} \partial_{P^{j^{\prime}}} \check{f}_{P, \sigma}^{1}\left(k_{1}\right) \ni-v_{\bar{\alpha}}^{\sigma}\left(k_{1}\right) R_{P, \sigma ; 1}\left(\Lambda_{P, \sigma}^{1}\right)^{j} R_{P, \sigma ; 1} R_{P, \sigma}\left(\Lambda_{P, \sigma}\right)^{j^{\prime}} \breve{\psi}_{P, \sigma}
$$

by the combined application of the first two identities in (1.15). As this term contains two resolvents not separated by $\Lambda_{P, \sigma}$, it is not directly tractable by the spectral ingredients (1.8), (1.9). We leave it open here if these latter estimates could be generalized so as to control (1.16). Given that the inductive proof of (1.9) in its present form extends over thirty pages, such a generalization would surely be a very formidable task. Fortunately, theory of non-commutative recurrence relations allows us to circumvent this problem, as we now explain.

We recall the dressing transformation $W_{P, \sigma}:=e^{b^{*}\left(f_{P, \sigma}\right)-b\left(f_{P, \sigma}\right)}$ from [Fr73], where

$$
f_{P, \sigma}(k):=\frac{v_{\bar{\alpha}}^{\sigma}(k)}{|k| \alpha_{P, \sigma}(\hat{k})}, \quad \alpha_{P, \sigma}(\hat{k}):=\left(1-\hat{k} \cdot \nabla E_{P, \sigma}\right), \quad \hat{k}=k /|k|
$$

and define the dressed operators $b_{W}(k):=W_{P, \sigma}^{*} b(k) W_{P, \sigma}=b(k)+f_{P, \sigma}(k)$. We also introduce the objects $\hat{f}_{P, \sigma}^{n}\left(k_{1}, \ldots, k_{n}\right):=b_{W}\left(k_{1}\right) \ldots b_{W}\left(k_{n}\right) \check{\psi}_{P, \sigma}$ which now replace $\check{f}_{P, \sigma}^{n}$ considered above. Since the functions $f_{P, \sigma}$ given by (1.17) are explicit and their derivatives w.r.t. $P$ up to second order 
have mild dependence on $\sigma$, it is clear from (1.12) that it suffices to analyse $\hat{f}_{P, \sigma}^{n}$. We show in Subsection 4.3 that the following recurrence relation holds true

$$
\begin{aligned}
\hat{f}_{P, \sigma}^{n}\left(k_{1}, \ldots, k_{n}\right)=(-) R_{P, \sigma ; n}\left(\sum_{i=1}^{n} F_{P, \sigma}\left(k_{i}, k_{1}+\ldots \check{i} \ldots+k_{n}\right) \hat{f}_{P, \sigma}^{n-1}\left(k_{1}, \ldots \check{i} \ldots, k_{n}\right)\right. & \\
& \left.+\sum_{1 \leq i<i^{\prime} \leq n} g_{P, \sigma}\left(k_{i}\right) \cdot g_{P, \sigma}\left(k_{i^{\prime}}\right) \hat{f}_{P, \sigma}^{n-2}\left(k_{1}, \ldots \check{i} \ldots \check{i^{\prime}} \ldots, k_{n}\right)\right)
\end{aligned}
$$

with the initial conditions $\hat{f}_{P, \sigma}^{0}=\breve{\psi}_{P, \sigma}, \hat{f}_{P, \sigma}^{1}\left(k_{1}\right)=(-) R_{P, \sigma ; 1} F_{P, \sigma}\left(k_{1}, 0\right) \check{\psi}_{P, \sigma}$ and the definitions

$$
F_{P, \sigma}(\tilde{k}, k):=-g_{P, \sigma}(\tilde{k}) \cdot\left(\Lambda_{P, \sigma}+k+\frac{1}{2} \tilde{k}\right), \quad g_{P, \sigma}(k):=f_{P, \sigma}(k) k
$$

Formula (1.18) can be seen as a recurrence relation of the form (1.1) with $\hat{a}, \hat{b} \neq 0$. From (1.3) we obtain the the following representation of the solution:

$$
\begin{aligned}
\hat{f}_{P, \sigma}^{n}= & n ! P_{\mathrm{sym}} \sum_{\ell=0}^{[n / 2]} \sum_{2 \leq i_{1} \ll \cdots \ll i_{\ell} \leq n} \frac{(-1)^{\ell}}{2^{\ell}} g_{P, \sigma ; 1} \ldots g_{P, \sigma ; n} \times \\
& \times\left(R_{P, \sigma ; i_{1}} \hat{\partial}_{i_{1}} \hat{\partial}_{i_{1}-1}\right) \ldots\left(R_{P, \sigma ; i_{\ell}} \hat{\partial}_{i_{\ell}} \hat{\partial}_{i_{\ell}-1}\right)\left\{\left(R_{P, \sigma ; n} \Lambda_{P, \sigma ; n}\right) \ldots\left(R_{P, \sigma ; 1} \Lambda_{P, \sigma ; 1}\right)\right\} \breve{\psi}_{P, \sigma},
\end{aligned}
$$

where $\Lambda_{P, \sigma ; i}:=\Lambda_{P, \sigma}+\underline{k}_{i-1}+\frac{1}{2} k_{i}$, the notation $i \ll i^{\prime}$ means $i+1<i^{\prime}$, and each operator $\left(R_{P, \sigma ; i} \hat{\partial}_{i} \hat{\partial}_{i-1}\right)$ acts on the product in the curly bracket by replacing $\left(R_{P, \sigma ; i} \Lambda_{P, \sigma ; i}\right)\left(R_{P, \sigma ; i-1} \Lambda_{P, \sigma ; i-1}\right)$ with $R_{P, \sigma ; i}$.

Let us now compare formulas (1.20) and (1.14). First, by (1.17), (1.19), $g_{P, \sigma}$ and $v_{\bar{\alpha}}^{\sigma}$ have very similar behaviour at small $k$. Second, the summation over $\ell$ and $i_{1}, \ldots, i_{\ell}$ merely leads to an additional factor $c^{n}$, which is allowed in (1.11). Thus the main difference are the factors $\Lambda_{P, \sigma ; i}$ present in formula (1.20) even before computing the derivatives. We note that the term $\ell=0$ has the desirable alternating structure (i.e. resolvents separated by $\Lambda_{P, \sigma}$ ) which is needed to use the spectral bounds (1.8), (1.9). However, for $\ell>0$ this structure can be disturbed by the action of the operators $\left(R_{P, \sigma ; i} \hat{\partial}_{i} \hat{\partial}_{i-1}\right)$ which create terms with resolvents not separated by $\Lambda_{P, \sigma}$. The key point and the essence of the method is that the total number of resolvents in such terms is strictly less than $n$. This is due to the fact that $\left(R_{P, \sigma ; i} \hat{\partial}_{i} \hat{\partial}_{i-1}\right)$ effectively removes one resolvent. This missing resolvent can then be replaced by the additional resolvent coming from $\partial_{P^{j}} R_{P, \sigma ; i}=R_{P, \sigma ; i}\left(\Lambda_{P, \sigma}^{i}\right)^{j} R_{P, \sigma ; i}$ so that simple bounds $\left\|R_{P, \sigma ; i}\right\| \leq c|k|_{i}^{-1}$ lead to the correct number of factors $\left|k_{i^{\prime}}\right|^{-1}$ as required in (1.11). We show in Subsection 6.2 that this mechanism works in all the relevant cases.

For the sake of clarity, we illustrate this mechanism by a simple example. Let us consider the following contribution to $\partial_{P^{j}} \partial_{P^{\prime}} \hat{f}_{P, \sigma}^{2}$

$$
\begin{aligned}
\partial_{P^{j}} \partial_{P j^{\prime}} \hat{f}_{P, \sigma}^{2} & \ni(-1) P_{\mathrm{sym}} g_{P, \sigma ; 1} g_{P, \sigma ; 2}\left(\partial_{P^{j}} R_{P, \sigma ; 2} \hat{\partial}_{2} \hat{\partial}_{1}\right)\left\{\left(R_{P, \sigma ; 2} \Lambda_{P, \sigma ; 2}\right)\left(R_{P, \sigma ; 1} \Lambda_{P, \sigma ; 1}\right)\right\} \partial_{P j^{\prime}} \breve{\psi}_{P, \sigma} \\
& =(-1) P_{\mathrm{sym}} g_{P, \sigma ; 1} g_{P, \sigma ; 2}\left\{\partial_{P^{j}} R_{P, \sigma ; 2}\right\} \partial_{P^{j^{\prime}}} \check{\psi}_{P, \sigma} \\
& =(-1) P_{\mathrm{sym}} g_{P, \sigma ; 1} g_{P, \sigma ; 2}\left\{R_{P, \sigma ; 2}\left(\Lambda_{P, \sigma}^{2}\right)^{j} R_{P, \sigma ; 2}\right\} R_{P, \sigma}\left(\Lambda_{P, \sigma}\right)^{j^{\prime}} \breve{\psi}_{P, \sigma},
\end{aligned}
$$

where in the last step we applied (1.15). This expression has a structure similar to (1.16), but the essential difference is that it is a contribution to the two-photon and not the one-photon wave 
function. Thus using the simple resolvent bounds $\left\|R_{P, \sigma ; 2}\left(\Lambda_{P, \sigma}^{2}\right)^{j}\right\|,\left\|R_{P, \sigma ; 2}\right\| \leq c|\underline{k}|_{2}^{-1}$, the facts that $|\underline{k}|_{2}^{-1} \leq|\underline{k}|_{1}^{-1},\left\|R_{P, \sigma}\left(\Lambda_{P, \sigma}\right)^{j^{\prime}} \check{\psi}_{P, \sigma}\right\| \leq c \sigma^{-\delta_{\lambda_{0}}}$ and $\left|g_{P, \sigma}(k)\right| \leq c v_{\bar{\alpha}}^{\sigma}(k)$, we obtain in agreement with our main result (1.11)

$$
\|(1.21)\| \leq c \sigma^{-\delta_{\lambda_{0}}} \frac{v_{\bar{\alpha}}^{\sigma}\left(k_{1}\right)}{\left|k_{1}\right|} \frac{v_{\bar{\alpha}}^{\sigma}\left(k_{2}\right)}{\left|k_{2}\right|} .
$$

This paper is organized as follows: In Section 2 we list some preliminaries and state precisely our results. In Section 3 we develop the theory of non-commutative recurrence relations in a form suitable for our investigation. In Section 4 we use this theory to derive formulas (1.14), (1.20) for the ground state wave functions. In Section 5 we identify convenient sufficient conditions for the bounds (1.11) on the derivatives of the wave functions to hold. These conditions are then verified in Section 6. More standard parts of the discussion are postponed to the appendices.

Acknowledgment: A.P. thanks the Hausdorff Research Institute of Mathematics, Bonn, for hospitality. A.P. is supported by the NSF grant \#DMS-0905988.

W.D. thanks the University of California Davis and the Hausdorff Research Institute for Mathematics, Bonn, for hospitality. W.D. is supported by the German Research Foundation (DFG) within the grants SP181/25-2, DY107/1-1, DY107/2-1. Moreover, he would like to acknowledge the support of the Danish Council for Independent Research, grant no. 09-065927 "Mathematical Physics", and of the Lundbeck Foundation.

\section{Preliminaries and results}

In this section we list some preliminaries and state the main results of this paper which were announced already in Section 1.2 of [DP12].

Let $\mathfrak{h}_{\mathrm{fi}}=L^{2}\left(\mathbb{R}^{3}, d^{3} k\right)$ be the single-photon subspace in the fiber picture. Let $\Gamma\left(\mathfrak{h}_{\mathrm{fi}}\right)$ be the symmetric Fock space over $\mathfrak{h}_{\mathrm{fi}}$ and let us denote the corresponding (improper) creation and annihilation operators by $b^{*}(k)$ an $b(k)$. We will denote by $\kappa=1$ the ultraviolet cut-off and by $\sigma \in(0, \kappa]$ the infrared cut-off. The fiber Hamiltonian of the massless Nelson model with these cut-offs is a selfadjoint operator on a domain $D\left(H_{P, \sigma}\right) \subset \Gamma\left(\mathfrak{h}_{\mathrm{fi}}\right)$ and it is given by the formula

$$
H_{P, \sigma}:=\frac{1}{2}\left(P-P_{\mathrm{f}}\right)^{2}+H_{\mathrm{f}}+\int d^{3} k v_{\bar{\alpha}}^{\sigma}(k)\left(b(k)+b^{*}(k)\right),
$$

where $H_{\mathrm{f}}=\int d^{3} k|k| b^{*}(k) b(k), P_{\mathrm{f}}:=\int d^{3} k k b^{*}(k) b(k)$ are the photon total energy and momentum operators. By the Kato-Rellich theorem, we have $D\left(H_{P, \sigma}\right)=D\left(H_{P, \text { free }}\right)$, where $H_{P \text {,free }}$ is given by (2.1) with the interaction term omitted. To define the form factor $v_{\bar{\alpha}}^{\sigma}$ we need to introduce some notation: Let $\mathcal{B}_{r}$ be the open ball of radius $r>0$ centered at zero. Let $\chi_{r} \in C_{0}^{\infty}\left(\mathbb{R}^{3}\right)$ be a function which is rotationally invariant, non-increasing in the radial direction, supported in $\mathcal{B}_{r}$ and equal to one on $\mathcal{B}_{\left(1-\varepsilon_{0}\right) r}$, for $0<\varepsilon_{0}<1$. Let $\lambda \neq 0$ be the coupling constant. Then the form-factor is given by

$$
v_{\bar{\alpha}}^{\sigma}(k):=\lambda \frac{\chi_{[\sigma, k)}(k)|k|^{\bar{\alpha}}}{(2|k|)^{\frac{1}{2}}},
$$


where $\chi_{[\sigma, k)}(k):=\mathbf{1}_{\mathcal{B}_{\sigma}^{\prime}}(k) \chi_{k}(k)$ and $\mathcal{B}_{\sigma}^{\prime}=\mathbb{R}^{3} \backslash \mathcal{B}_{\sigma}$. The parameter $0 \leq \bar{\alpha} \leq 1 / 2$ was needed in [DP12] and is kept here only for consistency with this earlier work. We stress that all the results listed below remain true in the infrared singular case $\bar{\alpha}=0$.

As our analysis concerns the bottom of the spectrum of the fiber Hamiltonians, let us define

$$
E_{P, \sigma}:=\inf \sigma\left(H_{P, \sigma}\right),
$$

where $\sigma$ denotes the spectrum. Under certain conditions, stated in Theorem 2.1 below, $E_{P, \sigma}$ is a non-degenerate isolated eigenvalue and its normalized eigenvector is denoted by $\breve{\psi}_{P, \sigma}$. The phase of the vector $\breve{\psi}_{P, \sigma}$ is fixed by the definitions

$$
\check{\psi}_{P, \sigma}:=W_{P, \sigma}^{*} \check{\phi}_{P, \sigma}, \quad \check{\phi}_{P, \sigma}:=\frac{\oint_{\gamma} \frac{d w}{H_{P, \sigma}^{W}-w} \Omega}{\left\|\oint_{\gamma} \frac{d w}{H_{P, \sigma}^{W}-w} \Omega\right\|},
$$

where $H_{P, \sigma}^{W}:=W_{P, \sigma} H_{P, \sigma} W_{P, \sigma}^{*}$ with $W_{P, \sigma}$ defined in (4.9), $\Omega$ is the vacuum vector in $\Gamma\left(\mathfrak{h}_{\mathrm{fi}}\right)$, and $\gamma$ is a circle of integration enclosing no other point of the spectrum of $H_{P, \sigma}^{W}$ apart form $E_{P, \sigma}$; for details see Section 5 in [DP17]. Since we are interested in small values of the total momentum $P$ at which the electron moves slower than the photons, we will consider $P$ from the set

$$
S:=\left\{P \in \mathbb{R}^{3}|| P \mid<1 / 3\right\} .
$$

As we work in the weak coupling regime we restrict attention to $|\lambda| \in\left(0, \lambda_{0}\right]$, where $\lambda_{0}$ is sufficiently small as specified in Theorem 2.1 .

The following theorem collects some results of the companion paper [DP17], which are relevant for the present investigation. We remark that regularity of $P \mapsto E_{P, \sigma}$ in infrared singular models was studied before in particular in [AH12, KM12, FP10]. Analyticity of ground state projections was established in some infrared regular models in [FFS14]. However, to our knowledge, derivatives of ground state vectors and their dependence on the infrared cut-off was not treated in infrared singular models before [DP17].

Theorem 2.1. Fix $0 \leq \bar{\alpha} \leq 1 / 2$. Then there exists $\lambda_{0}>0$ s.t. for all $|\lambda| \in\left(0, \lambda_{0}\right]$ and $P \in S$, the following statements hold:

(a) $S \ni P \mapsto E_{P, \sigma}$ is analytic and strictly convex, for all $\sigma \in(0, \kappa]$. Moreover,

$$
\left|\partial_{P}^{\beta_{1}} E_{P, \sigma}\right| \leq c, \quad\left|\partial_{P}^{\beta_{2}} E_{P, \sigma}\right| \leq c, \quad\left|\partial_{P}^{\beta_{3}} E_{P, \sigma}\right| \leq c / \sigma^{\delta_{\lambda_{0}}}
$$

for multiindices $\beta_{\ell}$ s.t. $\left|\beta_{\ell}\right|=\ell, \ell \in\{1,2,3\}$.

(b) For $\sigma \in(0, \kappa], E_{P, \sigma}$ is a simple eigenvalue corresponding to a normalized eigenvector $\breve{\psi}_{P, \sigma}$, whose phase is fixed in (2.4). There holds

$$
\left\|\partial_{P}^{\beta} \breve{\psi}_{P, \sigma}\right\| \leq c / \sigma^{\delta_{\lambda_{0}}}
$$

for multiindices $\beta$ s.t. $0<|\beta| \leq 2$.

The constant $c$ above is independent of $\sigma, P, \lambda, \bar{\alpha}$ within the assumed restrictions. Clearly, all statements above remain true after replacing $\lambda_{0}$ by some $\tilde{\lambda}_{0} \in\left(0, \lambda_{0}\right]$. The resulting function $\tilde{\lambda}_{0} \mapsto \delta_{\tilde{\lambda}_{0}}$ can be chosen positive and s.t. $\lim _{\tilde{\lambda}_{0} \rightarrow 0} \delta_{\tilde{\lambda}_{0}}=0$. 
As mentioned in the introduction, the regularity of the vector $\breve{\psi}_{P, \sigma}$, established in Theorem 2.1, is not sufficient for scattering theory of two electrons. One also needs similar bounds for their wave functions $f_{P, \sigma}^{n}$. Clearly, each $f_{P, \sigma}^{n}$ is a square-integrable function symmetric in $n$ variables from $\mathbb{R}^{3}$. We introduce the following auxiliary functions:

$$
g_{\sigma}^{n}\left(k_{1}, \ldots, k_{n}\right):=\prod_{i=1}^{n} \frac{c \lambda \chi_{\left[\sigma, \kappa_{*}\right)}\left(k_{i}\right)\left|k_{i}\right|^{\bar{\alpha}}}{\left|k_{i}\right|^{3 / 2}}, \quad \kappa_{*}:=\left(1-\varepsilon_{0}\right)^{-1} \kappa,
$$

where $c$ is some positive constant independent of $n, \sigma, P$ and $\lambda$ within the restrictions specified above. We also introduce the notation

$$
\mathcal{A}_{r_{1}, r_{2}}:=\left\{k \in \mathbb{R}^{3}\left|r_{1}<\right| k \mid<r_{2}\right\}
$$

where $0 \leq r_{1}<r_{2}$. Now we are ready to state the required properties of the functions $f_{P, \sigma}^{n}$.

Theorem 2.2. Fix $0 \leq \bar{\alpha} \leq 1 / 2$. Then there exists $\lambda_{0}>0$ s.t. for all $|\lambda| \in\left(0, \lambda_{0}\right]$ and $P \in S$ the following statements hold:

(a) Let $\left\{f_{P, \sigma}^{n}\right\}_{n \in \mathbb{N}_{0}}$ be the n-particle components of $\check{\psi}_{P, \sigma}$ and let $\overline{\mathcal{A}}_{\sigma, \kappa}^{\times n}$ be defined as the Cartesian product of $n$ copies of the closure of the set $\mathcal{A}_{\sigma, k}$ introduced in (2.9). Then, for any $P \in S$, the function $f_{P, \sigma}^{n}$ is supported in $\overline{\mathcal{A}}_{\sigma, \kappa}^{\times n}$.

(b) The function

$$
S \times \mathcal{A}_{\sigma, \infty}^{\times n} \ni\left(P ; k_{1}, \ldots, k_{n}\right) \mapsto f_{P, \sigma}^{n}\left(k_{1}, \ldots, k_{n}\right)
$$

is twice continuously differentiable and extends by continuity, together with its derivatives, to the set $S \times \overline{\mathcal{A}}_{\sigma, \infty}^{\times n}$.

(c) For any multiindex $\beta, 0 \leq|\beta| \leq 2$, the function (2.10) satisfies

$$
\begin{aligned}
\left|\partial_{k_{l}}^{\beta} f_{P, \sigma}^{n}\left(k_{1}, \ldots, k_{n}\right)\right| & \leq \frac{1}{\sqrt{n !}}\left|k_{l}\right|^{-|\beta|} g_{\sigma}^{n}\left(k_{1}, \ldots, k_{n}\right), \\
\left|\partial_{P}^{\beta} f_{P, \sigma}^{n}\left(k_{1}, \ldots, k_{n}\right)\right| & \leq \frac{1}{\sqrt{n !}} \frac{1}{\sigma^{\delta_{\lambda_{0}}}} g_{\sigma}^{n}\left(k_{1}, \ldots, k_{n}\right), \\
\left|\partial_{P^{\prime}} \partial_{k_{l}^{j}} f_{P, \sigma}^{n}\left(k_{1}, \ldots, k_{n}\right)\right| & \leq \frac{1}{\sqrt{n !}} \frac{1}{\sigma^{\delta_{\lambda_{0}}}}\left|k_{l}\right|^{-1} g_{\sigma}^{n}\left(k_{1}, \ldots, k_{n}\right),
\end{aligned}
$$

where the function $\tilde{\lambda}_{0} \mapsto \delta_{\tilde{\lambda}_{0}}$ has the properties specified in Theorem 2.1, $g_{\sigma}^{n}$ is defined in (2.8) and the factor $\sigma^{-\delta_{\lambda_{0}}}$ in (2.12) can be omitted for $|\beta|=0$.

Parts (a), (b) and estimate (2.11) in (c) can be extracted from [Fr73, Fr74, Fr] or proven using methods from these papers. Estimate (2.12) for $|\beta|=1$ and (2.13) require only the standard formula (1.14) for the wave functions and the spectral ingredient (1.8). All these straightforward estimates are proven in Appendix A. The main part of the paper is devoted to the proof of (2.12) for $|\beta|=$ 2 which requires both spectral ingredients (1.8) and (1.9) and the novel formula for the wave functions (1.20). 


\section{Standing assumptions and conventions:}

1. The parameter $1 / 2 \geq \bar{\alpha} \geq 0$, which appeared in (2.2) is kept fixed in the remaining part of the paper.

2. The total momentum $P$ belongs to the set $S$ defined in (2.5). The photon momenta $k_{1}, \ldots, k_{n}$ take arbitrary values from $\mathbb{R}^{3}$.

3. The maximal value of the coupling constant $\lambda_{0}>0$ is sufficiently small. In particular s.t. Theorem 2.1 holds.

4. $\tilde{\lambda}_{0} \mapsto \delta_{\tilde{\lambda}_{0}}$ denotes a positive function of $\tilde{\lambda}_{0} \in\left(0, \lambda_{0}\right]$, which may differ from line to line and has the property $\lim _{\tilde{\lambda}_{0} \rightarrow 0} \delta_{\tilde{\lambda}_{0}}=0$. (Cf. Theorem 2.1).

5. We will denote by $c, c^{\prime}, c^{\prime \prime}$ numerical constants which may change from line to line.

6. We reserve letters $j, j^{\prime}=1,2,3$ to denote vector indices (as superscripts). Letters $i, i^{\prime}$ usually count photon momenta. E.g. $k_{1}^{j}, \ldots, k_{i}^{j}, \ldots k_{n}^{j}$.

\section{Notation related to non-commutative recurrence relations:}

1. $\mathrm{Op}_{\hat{\boldsymbol{a}}, \hat{\boldsymbol{b}}}^{(n)}$ is the quantization map defined in formula (3.4).

2. The operator $\hat{\delta}_{i}=: \hat{b}_{i+1, i} \hat{\partial}_{i+1} \hat{\partial}_{i}$ defined on the range of the quantization map appears for the first time below formula (3.5)

3. The symmetrization operator $P_{\text {sym }}$ is defined in (3.17).

4. For the operators $\hat{a}_{\mathrm{ns}}$ and $\hat{a}:=P_{\mathrm{sym}} \hat{a}_{\mathrm{ns}}$ see (3.18)-(3.19).

5. For $\left(R_{P, \sigma ; i+1} \hat{\partial}_{i+1} \hat{\partial}_{i}\right), C_{\ell}, C^{2 \ell}, \hat{\delta}_{C_{\ell}}, \hat{\delta}_{C_{\ell}}^{\prime}, \hat{\delta}_{C_{\ell}}^{\prime \prime}, I_{C_{\ell}}$ we refer to (4.57)-(4.63).

6. For $\alpha(n, \ldots, 1):=\mathrm{Op}_{\hat{\boldsymbol{a}}, \hat{\boldsymbol{b}}}^{(n)}\left(a_{n}, \ldots, a_{1}\right)=\left(R_{P, \sigma ; n} \Lambda_{P, \sigma ; n}\right) \ldots\left(R_{P, \sigma ; 1} \Lambda_{P, \sigma ; 1}\right)$, see (4.55), (4.56).

7. For $\partial_{R_{i}} I_{C_{\ell}}, \partial_{\Lambda_{i}} I_{C_{\ell}}$ and $\partial_{\breve{\psi}} I_{C_{\ell}}$ and second derivatives of this type see Section 5 .

8. $i \ll i^{\prime}$ means $i+1<i^{\prime}$.

\section{Notation related to the Nelson model:}

1. $b_{W}(k):=b(k)+f_{P, \sigma}(k)$, where $f_{P, \sigma}(k):=\frac{v_{\bar{\alpha}}^{\sigma}(k)}{|k| \alpha_{P, \sigma}(\hat{k})}, \alpha_{P, \sigma}(\hat{k}):=\left(1-\hat{k} \cdot \nabla E_{P, \sigma}\right)$ and $\hat{k}:=k /|k|$, appears in (4.5).

2. $g_{P, \sigma}(k):=f_{P, \sigma}(k) k$ appears in (4.49).

3. $\check{f}_{P, \sigma}^{m}\left(k_{1}, \ldots, k_{m}\right):=b\left(k_{1}\right) \ldots b\left(k_{m}\right) \check{\psi}_{P, \sigma}$ is introduced in (4.33).

4. $\hat{f}_{P, \sigma}^{m}\left(k_{1}, \ldots, k_{m}\right):=b_{W}\left(k_{1}\right) \ldots b_{W}\left(k_{m}\right) \breve{\psi}_{P, \sigma}$ is introduced in (4.42). 
5. Given $k_{1}, \ldots, k_{n}$ and a permutation $\pi \in S_{n}$ we write

$$
\begin{array}{ll}
\underline{k}_{i}:=k_{1}+\cdots+k_{i}, & \underline{k}_{\pi, i}:=k_{\pi(1)}+\cdots+k_{\pi(i)}, \\
|\underline{k}|_{i}:=\left|k_{1}\right|+\cdots+\left|k_{i}\right|, \quad|\underline{k}|_{\pi, i}:=\left|k_{\pi(1)}\right|+\cdots+\left|k_{\pi(i)}\right| .
\end{array}
$$

6. We use the following quantities:

$$
\begin{aligned}
& \Lambda_{P, \sigma}:=\nabla E_{P, \sigma}-\left(P-P_{\mathrm{f}}\right), \\
& \Lambda_{P, \sigma ; i}:=\Lambda_{P, \sigma}+\underline{k}_{i-1}+\frac{1}{2} k_{i}, \\
& \Lambda_{P, \sigma}^{i}:=\Lambda_{P, \sigma}+\underline{k}_{i}, \\
& F_{P, \sigma}(\tilde{k}, k):=-g_{P, \sigma}(\tilde{k}) \cdot\left(\Lambda_{P, \sigma}+k+\frac{1}{2} \tilde{k}\right),
\end{aligned}
$$

which appear in (4.8), (4.52), (5.6), (4.50) respectively.

7. We use the following quantities:

$$
\begin{aligned}
H_{P, \sigma ; k_{1}, \ldots, k_{n}} & :=H_{P-\left(k_{1}+\cdots+k_{n}\right), \sigma}+\left|k_{1}\right|+\cdots+\left|k_{n}\right|, \\
R_{P, \sigma ; k_{1}, \ldots, k_{n}} & :=\frac{1}{H_{P, \sigma ; k_{1}, \ldots, k_{n}}-E_{P, \sigma}}
\end{aligned}
$$

which appear in (4.34) and (4.35).

8. We use the following resolvents

$$
\begin{aligned}
R_{P, \sigma} & :=\frac{1}{H_{P, \sigma}-E_{P, \sigma}}, \\
R_{P, \sigma ; i} & :=\frac{1}{H_{P-\underline{k}_{i}, \sigma}-E_{P, \sigma}+|\underline{k}|_{i}}, \\
R_{P, \sigma}^{i} & :=\frac{1}{H_{P, \sigma}-E_{P, \sigma}+r(\underline{k})_{i}},
\end{aligned}
$$

where $r(\underline{k})_{i}:=|\underline{k}|_{i}+\left|\underline{k}_{i}\right|^{2} / 2-\nabla E_{P, \sigma} \cdot \underline{k}_{i}$. They appear in (6.1), (6.2), (6.3), respectively. We note that $R_{P, \sigma ; i}=R_{P, \sigma ; k_{1}, \ldots, k_{i}}$.

9. In Section 6 and Appendix A we drop subscripts $P, \sigma$ from most quantities considered above. For example $\Lambda^{i}:=\Lambda_{P, \sigma}^{i}$.

10. In Appendix A we consider the above quantities with permuted photon momenta. Then we write e.g. $\Lambda_{\pi}^{i}:=\Lambda_{P, \sigma}+\underline{k}_{\pi, i}$.

\section{Non-commutative recurrence relations}

\subsection{General theory}

Let $\boldsymbol{a}:=\left\{a_{i}\right\}_{i \in \mathbb{N}}, \boldsymbol{b}:=\left\{b_{i+1, i}\right\}_{i \in \mathbb{N}}$ be two sequences of real variables, $\operatorname{Pol}(\boldsymbol{a}, \boldsymbol{b})$ be the commutative algebra of all polynomials in $\boldsymbol{a}$ and $\boldsymbol{b}$ and $\operatorname{Pol}(\boldsymbol{a})$ the algebra of polynomials in $\boldsymbol{a}$. We denote by 
$\partial_{i}:=\partial / \partial_{a_{i}}$ the operators on $\operatorname{Pol}(\boldsymbol{a}, \boldsymbol{b})$ acting by differentiation w.r.t. $a_{i}$. (Derivatives w.r.t. $b_{i+1, i}$ will not be used). We also define the following operators on $\operatorname{Pol}(\boldsymbol{a}, \boldsymbol{b})$ for $i \in \mathbb{N}$

$$
\delta_{i}:=b_{i+1, i} \partial_{i+1} \partial_{i}
$$

where $b_{i+1, i}$ is understood as a multiplication operator. Furthermore, we define the following subspaces of $\operatorname{Pol}(\boldsymbol{a}, \boldsymbol{b})$ for $n \in \mathbb{N}$

$$
\widetilde{\operatorname{Pol}}^{(n)}(\boldsymbol{a}, \boldsymbol{b}):=\operatorname{Span}\left\{\delta_{n}^{\mu_{n}} \delta_{n-1}^{\mu_{n-1}} \ldots \delta_{1}^{\mu_{1}}\left(a_{n} a_{n-1} \ldots a_{1}\right) \mid \mu_{i} \in\{0,1\}\right\}
$$

where $\delta_{j}^{\mu_{j}}=\delta_{j}$ if $\mu_{j}=1$, and $\delta_{j}^{\mu_{j}}=1$ if $\mu_{j}=0$. We note that $\widetilde{\mathrm{Pol}}^{(n)}(\boldsymbol{a}, \boldsymbol{b})$ is spanned by monomials of the form

$$
a_{n} \ldots b_{i+1, i} \ldots b_{i^{\prime}+1, i^{\prime}} \ldots a_{1}
$$

which arise by removing pairs $a_{i+1} a_{i}$ from the monomial $a_{n} \ldots a_{1}$ and replacing them with $b_{i+1, i}$.

We can treat $\widetilde{\operatorname{Pol}}(\boldsymbol{a}, \boldsymbol{b})$ as a class of polynomial symbols and define their quantization as follows: Let $\left\{X_{i}\right\}_{i \in \mathbb{N}_{0}}$ be a family of vector spaces and $L\left(X_{i}, X_{j}\right)$ is the space of linear maps from $X_{i}$ to $X_{j}$. Let $\hat{\boldsymbol{a}}:=\left\{\hat{a}_{i}\right\}_{i \in \mathbb{N}}, \hat{\boldsymbol{b}}:=\left\{\hat{b}_{i+1, i}\right\}_{i \in \mathbb{N}}$ be two sequences of linear operators $\hat{a}_{i}: X_{i-1} \rightarrow X_{i}$, $\hat{b}_{i+1, i}: X_{i-1} \rightarrow X_{i+1}$. We define the linear map $\operatorname{Op}_{\hat{\boldsymbol{a}}, \hat{\boldsymbol{b}}}^{(n)}: \widetilde{\operatorname{Pol}}^{(n)}(\boldsymbol{a}, \boldsymbol{b}) \rightarrow L\left(X_{0}, X_{n}\right)$ by the following specification on monomials

$$
\mathrm{Op}_{\hat{\boldsymbol{a}}, \hat{\boldsymbol{b}}}^{(n)}\left[a_{n} \ldots b_{i+1, i} \ldots b_{i^{\prime}+1, i^{\prime}} \ldots a_{1}\right]=\hat{a}_{n} \ldots \hat{b}_{i+1, i} \ldots \hat{b}_{i^{\prime}+1, i^{\prime}} \ldots \hat{a}_{1} .
$$

The case with $X_{i}=X, \hat{a}_{i}=\hat{a}$ and $\hat{b}_{i+1, i}=\hat{b}$ is of particular importance. In this case we simply denote the quantization map by $\mathrm{Op}_{\hat{a}, \hat{b}}^{(n)}$.

Clearly, various operations on $\overparen{\operatorname{Pol}}(\boldsymbol{a}, \boldsymbol{b})$ naturally carry over to operators from the ranges of the quantization maps. For example, we can define

$$
\hat{\delta}_{i} \mathrm{Op}_{\hat{\boldsymbol{a}}, \hat{\boldsymbol{b}}}[p(\boldsymbol{a}, \boldsymbol{b})]:=\mathrm{Op}_{\hat{\boldsymbol{a}}, \boldsymbol{b}}\left[\delta_{i} p(\boldsymbol{a}, \boldsymbol{b})\right], \quad p(\boldsymbol{a}, \boldsymbol{b}) \in \widetilde{\operatorname{Pol}}^{(n)}(\boldsymbol{a}, \boldsymbol{b}) .
$$

If there is a need for more explicit notation, we will write $\hat{\delta}_{i}=: \hat{b}_{i+1, i} \hat{\partial}_{i+1} \hat{\partial}_{i}$. We note, however, that $\hat{\partial}_{i}$ is not well defined, since $\partial_{i}$ does not leave $\widetilde{\operatorname{Pol}}^{(n)}(\boldsymbol{a}, \boldsymbol{b})$ invariant.

Now we can prove the main result of this section:

Theorem 3.1. Let $X$ be a vector space, $\left\{x_{n}\right\}_{n \in \mathbb{N}_{0}}$ a sequence of vectors from $X$ and $\hat{a}, \hat{b} \in L(X)$. Suppose the following recurrence relation holds

$$
x_{n}=\hat{a} x_{n-1}+\hat{b} x_{n-2}
$$

with the initial conditions $x_{0}$ and $x_{1}=\hat{a} x_{0}$. Then, for $n \geq 1$

$$
x_{n}=\mathrm{Op}_{\hat{a}, \hat{b}}^{(n)}\left[\exp \left(\sum_{i=1}^{n-1} b_{i+1, i} \partial_{i+1} \partial_{i}\right) a_{n} \ldots a_{1}\right] x_{0},
$$

where $\exp$ is defined by its power series and for $n=1$ the sum in the exponent above is equal to zero by convention. (We recall that the symbol $\mathrm{Op}_{\hat{a}, \hat{b}}^{(n)}$ implies that all the $\hat{a}_{n}$ are equal to $\hat{a}$ and all $\hat{b}_{i+1, i}$ are equal to $\hat{b}$.) 
Proof. We asume $n \geq 2$, write as before $\delta_{i}:=b_{i+1, i} \partial_{i+1} \partial_{i}$ and set $d_{n-1}:=\sum_{i=1}^{n-1} \delta_{i}$. Now we compute

$$
\begin{aligned}
\exp \left(d_{n-1}\right) a_{n} \ldots a_{1} & =\left\{\exp \left(d_{n-2}\right) \exp \left(\delta_{n-1}\right) a_{n} a_{n-1} \ldots a_{1}\right\} \\
& =\left\{\exp \left(d_{n-2}\right) a_{n} a_{n-1} \ldots a_{1}\right\}+\left\{\exp \left(d_{n-2}\right)\left(\delta_{n-1}\right) a_{n} a_{n-1} \ldots a_{1}\right\} \\
& =a_{n}\left\{\exp \left(d_{n-2}\right) a_{n-1} \ldots a_{1}\right\}+b_{n, n-1}\left\{\exp \left(d_{n-3}\right) a_{n-2} \ldots a_{1}\right\}
\end{aligned}
$$

Here in the second step we used that $\left(\delta_{n-1}\right)^{2} a_{n} a_{n-1} \ldots a_{1}=0$ and in the last step we could replace $d_{n-2}$ with $d_{n-3}$ since $\delta_{n-2} a_{n-2} \ldots a_{n}=0$. (For $n=2$ we have $a_{n-2} \ldots a_{n} \equiv 1$ by convention). Relation (3.8) gives

$$
\mathrm{Op}_{\hat{a}, \hat{b}}^{(n)}\left[\exp \left(d_{n-1}\right) a_{n} \ldots a_{1}\right]=\hat{a} \mathrm{Op}_{\hat{a}, \hat{b}}^{(n-1)}\left[\exp \left(d_{n-2}\right) a_{n-1} \ldots a_{1}\right]+\hat{b} \mathrm{Op}_{\hat{a}, \hat{b}}^{(n-2)}\left[\exp \left(d_{n-3}\right) a_{n-2} \ldots a_{1}\right]
$$

Acting with both sides of (3.9) on $x^{0}$ we obtain the recurrence relation (3.6). With the specified initial conditions it determines $x_{n}, n \geq 2$, uniquely.

In the next lines, we derive a variant of formula (3.7) which is convenient for our applications. Still using the notation $\delta_{i}:=b_{i+1, i} \partial_{i+1} \partial_{i}$ and the property $\left(\delta_{i}\right)^{2}=0$, we observe that the multinomial formula implies

$$
\left(\sum_{i=1}^{n-1} \delta_{i}\right)^{\ell} a_{n} \ldots a_{1}=\sum_{\mu:|\mu|=\ell} \frac{\ell !}{\mu !} \delta^{\mu} a_{n} \ldots a_{1}
$$

where $\mu:=(\mu(1), \ldots, \mu(n-1))$ is an $n-1$-index, $\mu !=\mu(1) ! \ldots \mu(n-1) !,|\mu|=\sum_{i=1}^{n-1} \mu(i)$ and $\delta^{\mu}:=\delta_{1}^{\mu(1)} \ldots \delta_{n-1}^{\mu(n-1)}$. Now we note that $\delta_{i}^{2}\left(a_{n} \ldots a_{1}\right)=0$, thus only for $\mu(i) \in\{0,1\}$ the corresponding terms can be non-zero.

Next, since $\delta_{i}^{2}\left(a_{n} \ldots a_{1}\right)=0$ and $\delta_{i} \delta_{i+1}\left(a_{n} \ldots a_{1}\right)=0$ we can write

$$
x_{n}=\sum_{\ell=0}^{[n / 2]} \frac{1}{\ell !} \mathrm{Op}_{\hat{a}, \hat{b}}^{(n)}\left[\left(\sum_{i=1}^{n-1} \delta_{i}\right)^{\ell} a_{n} \ldots a_{1}\right] x_{0} .
$$

Hence (3.11), (3.10) give

$$
x_{n}=\sum_{\ell=0}^{[n / 2]} \sum_{\mu:|\mu|=\ell} \mathrm{Op}_{\hat{a}, \hat{b}}^{(n)}\left[(\delta)^{\mu} a_{n} \ldots a_{1}\right] x_{0}=\sum_{\ell=0}^{[n / 2]} \sum_{1 \leq i_{1} \ll \cdots \ll i_{\ell} \leq n-1} \operatorname{Op}_{\hat{a}, \hat{b}}^{(n)}\left[\delta_{i_{1}} \ldots \delta_{i_{\ell}} a_{n} \ldots a_{1}\right] x_{0},
$$

where $i \ll i^{\prime}$ means $i+1<i^{\prime}$.

Now we consider a class of non-commutative recurrence relations for which the solution can be expressed in terms of the generalized framework in (3.4). Suppose that $X=\bigoplus_{i \in \mathbb{N}_{0}} X_{i}$, set $\hat{a}_{i}:=\left(\hat{a} \uparrow X_{i-1}\right), \hat{a}_{i}:=\left(\hat{b} \uparrow X_{i-1}\right)$ and assume that $\hat{a}_{i}: X_{i-1} \rightarrow X_{i}, \hat{b}_{i+1, i}: X_{i-1} \rightarrow X_{i+1}$ and $x_{0} \in X_{0}$. Thus setting $\hat{\boldsymbol{a}}:=\left\{\hat{a}_{i}\right\}_{i \in \mathbb{N}}, \hat{\boldsymbol{b}}:=\left\{\hat{b}_{i+1, i}\right\}_{i \in \mathbb{N}}$ we have

$$
x_{n}=\sum_{\ell=0}^{[n / 2]} \sum_{1 \leq i_{1} \ll \cdots \ll i_{\ell} \leq n-1} \hat{\delta}_{i_{1}} \ldots \hat{\delta}_{i_{\ell}} \mathrm{Op}_{\hat{\boldsymbol{a}}, \hat{\boldsymbol{b}}}^{(n)}\left[a_{n} \ldots a_{1}\right] x_{0} .
$$




\subsection{Fock space combinatorics}

The formula in (3.13) will be applied to the recurrence relation derived in (4.51) for the vectors $\hat{f}_{P, \sigma}^{n}\left(k_{1}, \ldots, k_{n}\right)$ by which we express the ground state wave functions $f_{P, \sigma}^{n}\left(k_{1}, \ldots, k_{n}\right)$ in (4.43). For the given sequence, it is not immediate to identify the families of symmetrized (in the variables $k_{1}, \ldots, k_{n}$ ) operators $\hat{a}_{i}$ and $\hat{b}_{i+1, i}$ that enter the solution according to the formula in (3.13). To this end, it is convenient to start from non-symmetrized operators acting on non-symmetric tensor products of Hilbert spaces which are related to $\hat{a}_{i}$ and $\hat{b}_{i+1, i}$. The definition of the non-symmetrized operators is dictated by the specific recurrence relation in (4.51) though in our discussion below this is not made explicit.

We start with an elementary observation, which will be important in the later part of this section.

Lemma 3.2. Let $f$ be a function of $m$ variables $k_{1}, \ldots, k_{m} \in \mathbb{R}^{3}$ with values in some set $Y$. Denote its symmetrization in $m^{\prime}$ consecutive variables $k_{i_{1}}, \ldots, k_{i_{m^{\prime}}}$ by

$$
\left[f\left(k_{1}, \ldots, k_{m}\right)\right]_{\mathrm{sym}}^{\left(i_{1}, i_{m^{\prime}}\right)}:=\frac{1}{m^{\prime} !} \sum_{\pi \in S_{m^{\prime}}} f\left(k_{1}, \ldots, k_{\pi\left(i_{1}\right)}, \ldots, k_{\pi\left(i_{m^{\prime}}\right)}, \ldots, k_{m}\right),
$$

where $S_{m^{\prime}}$ is the set of permutations of $m^{\prime}$ variables. Then

$$
\left[\left[f\left(k_{1}, \ldots, k_{m}\right)\right]_{\mathrm{sym}}^{\left(i_{1}, i_{m^{\prime}}\right)}\right]_{\mathrm{sym}}=\left[f\left(k_{1}, \ldots, k_{m}\right)\right]_{\mathrm{sym}},
$$

where $[\ldots]_{\mathrm{sym}}:=[\ldots]_{\mathrm{sym}}^{(1, m)}$.

Proof. We write

$$
\begin{aligned}
{\left[\left[f\left(k_{1}, \ldots, k_{m}\right)\right]_{\mathrm{sym}}^{\left(i_{1}, i_{m^{\prime}}\right)}\right]_{\mathrm{sym}} } & =\frac{1}{m^{\prime} !} \sum_{\pi \in S_{m^{\prime}}} \frac{1}{m !} \sum_{\rho \in S_{m}} f\left(k_{\rho(1)}, \ldots, k_{\rho\left(\pi\left(i_{1}\right)\right)}, \ldots, k_{\rho\left(\pi\left(i_{m^{\prime}}\right)\right)}, \ldots, k_{\rho(m)}\right) \\
& =\frac{1}{m^{\prime} !} \sum_{\pi \in S_{m^{\prime}}} \frac{1}{m !} \sum_{\rho_{\pi}^{\prime} \in S_{m}} f\left(k_{\rho_{\pi}^{\prime}(1)}, \ldots, k_{\rho_{\pi}^{\prime}\left(i_{1}\right)}, \ldots, k_{\rho_{\pi}^{\prime}\left(i_{m^{\prime}}\right)}, \ldots, k_{\rho_{\pi}^{\prime}(m)}\right) \\
& =\left[\left[f\left(k_{1}, \ldots, k_{m}\right)\right]_{\mathrm{sym}}\right]_{\mathrm{sym}}^{\left(i_{1}, i_{m^{\prime}}\right)} \\
& =\left[f\left(k_{1}, \ldots, k_{m}\right)\right]_{\mathrm{sym}},
\end{aligned}
$$

where we set $\rho_{\pi}^{\prime}=\rho \circ \tilde{\pi}$ and $\tilde{\pi}$ is the extension of $\pi$ from $\left(i_{1}, \ldots, i_{m^{\prime}}\right)$ to $(1, \ldots, m)$ by the identity.

Let $\mathcal{H}$ be a Hilbert space, $\Gamma_{\mathrm{ns}}\left(L^{2}\left(\mathbb{R}^{3}\right)\right):=\bigoplus_{m \in \mathbb{N}_{0}} L^{2}\left(\mathbb{R}^{3}\right)^{\otimes m}$ be the non-symmetrized Fock space and $X_{\mathrm{ns}}:=\Gamma_{\mathrm{ns}}\left(L^{2}\left(\mathbb{R}^{3}\right)\right) \otimes \mathcal{H}$. The elements of $X_{\mathrm{ns}}$ have the form $x_{\mathrm{ns}}=\left\{x_{\mathrm{ns}}^{m}\right\}_{m \in \mathbb{N}_{0}}$, where $x_{\mathrm{ns}}^{m}$ are $\mathcal{H}$-valued square integrable functions of $m$ variables from $\mathbb{R}^{3}$. Let $P_{\text {sym }}$ be the symmetrization operator on $X_{\mathrm{ns}}$ given by

$$
\left(P_{\mathrm{sym}} x_{\mathrm{ns}}\right)^{m}\left(k_{1}, \ldots, k_{m}\right)=\left[x_{\mathrm{ns}}^{m}\left(k_{1}, \ldots, k_{m}\right)\right]_{\mathrm{sym}}, \quad x_{\mathrm{ns}} \in X_{\mathrm{ns}} .
$$

The symmetric Fock space is given by $\Gamma\left(L^{2}\left(\mathbb{R}^{3}\right)\right):=\bigoplus_{m \in \mathbb{N}_{0}} P_{\text {sym }} L^{2}\left(\mathbb{R}^{3}\right)^{\otimes m}$ and $X:=\Gamma\left(L^{2}\left(\mathbb{R}^{3}\right)\right) \otimes \mathcal{H}$. The elements of $X$ will be denoted by $x=\left\{x^{m}\right\}_{m \in \mathbb{N}_{0}}$ and we treat $X$ as a subspace of $X_{\mathrm{ns}}$. We denote by $X_{\mathrm{ns}, \mathrm{in}}, X_{\mathrm{fin}}$ dense subspaces of $X_{\mathrm{ns}}, X$, respectively, consisting of vectors with only a finite number of non-zero Fock space components. 
Let $\hat{a}_{\mathrm{ns}}=\left\{\hat{a}_{\mathrm{ns}, m}\right\}_{m \geq r}$ be a sequence of bounded functions of $3 m$ real variables with values in $B(\mathcal{H})$, supported in some compact sets $C_{m} \subset \mathbb{R}^{3 m}$ and norm continuous in their interiors. It defines an operator on $X_{\mathrm{ns}, \text { fin }}$ as follows

$$
\begin{aligned}
& \left(\hat{a}_{\mathrm{ns}} x_{\mathrm{ns}}\right)^{m}\left(k_{1}, \ldots, k_{m}\right):=\hat{a}_{\mathrm{ns}, m}\left(k_{1}, \ldots, k_{m-r}, k_{m-r+1}, \ldots, k_{m}\right) x_{\mathrm{ns}}^{m-r}\left(k_{1}, \ldots, k_{m-r}\right) \text { for } m \geq r, \\
& \left(\hat{a}_{\mathrm{ns}} x_{\mathrm{ns}}\right)^{m}\left(k_{1}, \ldots, k_{m}\right):=0 \text { for } m<r .
\end{aligned}
$$

Similarly we define $\hat{a}:=P_{\text {sym }} \hat{a}_{\mathrm{ns}}$ as an operator on $X$ and call $r \in \mathbb{N}_{0}$ the order of the operators $\hat{a}_{\mathrm{ns}}, \hat{a}$. The following fact will be helpful for identifying $\hat{a}, \hat{a}_{\mathrm{ns}}$ in examples.

Lemma 3.3. Let $\hat{a}_{\mathrm{ns}}$ be an operator on $X_{\mathrm{ns}}$ of order $r$ defined as in (3.18)-(3.19). Suppose that the functions $\hat{a}_{\mathrm{ns}, m}$ are symmetric in the collections of variables $\left(k_{1}, \ldots, k_{m-r}\right)$ and $\left(k_{m-r+1}, \ldots, k_{m}\right)$ separately. Then

$$
\begin{gathered}
(\hat{a} x)^{m}\left(k_{1}, \ldots, k_{m}\right)=\frac{(m-r) ! r !}{m !} \sum_{\substack{1 \leq i_{1}<\cdots<i_{r} \leq m\\
}} \hat{a}_{\mathrm{ns}, m}\left(k_{1}, \ldots \check{i_{1}} \ldots . \check{i_{r}} \ldots, k_{m} ; k_{i_{1}}, \ldots, k_{i_{r}}\right) \times \\
\quad \times x^{m-r}\left(k_{1}, \ldots \check{i_{1}} \ldots . \check{i_{r}} \ldots, k_{m}\right)
\end{gathered}
$$

where we separated the two groups of variables by a semicolon for clarity.

Proof. We write

$$
\begin{aligned}
& (\hat{a} x)^{m}\left(k_{1}, \ldots, k_{m}\right)=\frac{1}{m !} \sum_{\pi \in S_{m}} \hat{a}_{\mathrm{ns}, m}\left(k_{\pi(1)}, \ldots, k_{\pi(m-r)} ; k_{\pi(m-r+1)}, \ldots, k_{\pi(m)}\right) x^{m-r}\left(k_{\pi(1)}, \ldots, k_{\pi(m-r)}\right)
\end{aligned}
$$

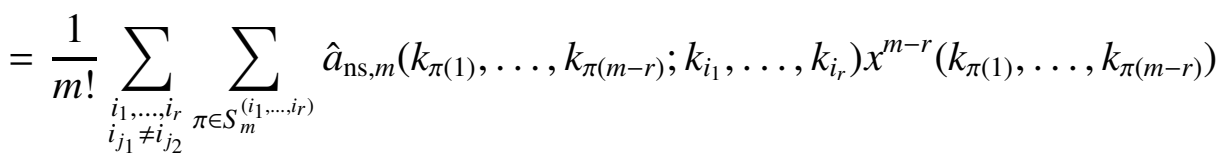

$$
\begin{aligned}
& =\frac{(m-r) !}{m !} \sum_{\substack{i_{1}, \ldots, i_{r} \\
i_{j_{1}} \neq i_{j_{2}}}} \hat{a}_{\mathrm{ns}, m}\left(k_{1}, \ldots \check{i_{1}} \ldots . \check{i_{r}} \ldots, k_{m} ; k_{i_{1}}, \ldots, k_{i_{r}}\right) \times \\
& \times x^{m-r}\left(k_{1}, \ldots \check{i_{1}} \ldots . \check{i_{r}} \ldots, k_{m}\right) \\
& =\frac{(m-r) ! r !}{m !} \sum_{1 \leq i_{1}<\cdots<i_{r} \leq m} \hat{a}_{\mathrm{ns}, m}\left(k_{i_{1}}, \ldots, k_{i_{r}} ; k_{1}, \ldots \check{i_{1}} \ldots . \check{i_{r}} \ldots, k_{m}\right) \\
& \times x^{m-r}\left(k_{1}, \ldots \check{i_{1}} \ldots . \check{\check{i_{r}}} \ldots, k_{m}\right),
\end{aligned}
$$

where $S_{m}^{\left(i_{1}, \ldots i_{r}\right)}$ is the set of permutations from $S_{m}$ such that $\pi(m-r+1)=i_{1}, \ldots, \pi(m)=i_{r}$.

\subsection{Non-commutative recurrence relations on Fock space}

Given a family $\hat{a}_{\mathrm{ns}}^{(f)}, \ldots, \hat{a}_{\mathrm{ns}}^{(1)}$ of operators defined in (3.18)-(3.19), with orders $r_{f}, \ldots, r_{1}$, respectively, using the definitions in (3.18) and (3.19) we have by iteration

$$
\begin{aligned}
& \left(\hat{a}_{\mathrm{ns}}^{(f)} \ldots \hat{a}_{\mathrm{ns}}^{(1)} x_{\mathrm{ns}}\right)^{m}\left(k_{1}, \ldots, k_{m}\right)=\hat{a}_{\mathrm{ns}, m}^{(f)}\left(k_{1}, \ldots, k_{m}\right)\left(\hat{a}_{\mathrm{ns}}^{(f-1)} \ldots \hat{a}_{\mathrm{ns}}^{(1)} x_{\mathrm{ns}}\right)^{m-r_{f}}\left(k_{1}, \ldots, k_{m-r_{f}}\right) \\
& \begin{array}{r}
\hat{a}_{\mathrm{ns}, m}^{(f)}\left(k_{1}, \ldots, k_{m}\right) \hat{a}_{\mathrm{ns}, m-r_{f}}^{(f-1)}\left(k_{1}, \ldots, k_{m-r_{f}}\right) \ldots \hat{a}_{\mathrm{ns}, m-\left(r_{2}+\cdots+r_{f}\right)}^{(1)}\left(k_{1}, \ldots, k_{m-\left(r_{2}+\cdots+r_{f}\right)}\right) \times \\
\times x_{\mathrm{ns}}^{m-\left(r_{1}+\cdots+r_{f}\right)}\left(k_{1}, \ldots, k_{m-\left(r_{1}+\cdots+r_{f}\right)}\right),
\end{array}
\end{aligned}
$$


for $m \geq\left(r_{1}+\cdots+r_{f}\right)$ and zero otherwise. Next, for $\hat{a}^{(j)}:=P_{\mathrm{sym}} \hat{a}_{\mathrm{ns}}^{(j)}$ we have by Lemma 3.2 and formula (3.22)

$$
\hat{a}^{(f)} \ldots \hat{a}^{(1)} x=P_{\mathrm{sym}} \hat{a}_{\mathrm{ns}}^{(f)} \ldots \hat{a}_{\mathrm{ns}}^{(1)} x, \quad x \in X .
$$

Let us now write formula (3.22) in the case $x_{\mathrm{ns}}=\left(x_{\mathrm{ns}}^{0}, 0, \ldots\right)=: x_{0}$. (We skip the index ns, since symmetrization acts trivially on this vector). Then there is only one non-trivial component, namely the one corresponding to $m=\left(r_{1}+\cdots+r_{f}\right)$ :

$$
\begin{aligned}
& \left(\hat{a}_{\mathrm{ns}}^{(f)} \ldots \hat{a}_{\mathrm{ns}}^{(1)} x_{0}\right)^{m}\left(k_{1}, \ldots, k_{m}\right) \\
& =\hat{a}_{\mathrm{ns}, r_{1}+\cdots+r_{f}}^{(f)}\left(k_{1}, \ldots, k_{r_{1}+\cdots+r_{f}}\right) \ldots \hat{a}_{\mathrm{ns}, r_{1}+\cdots+r_{j}}^{(j)}\left(k_{1}, \ldots, k_{r_{1}+\cdots+r_{j}}\right) \ldots \hat{a}_{\mathrm{ns}, r_{1}}^{(1)}\left(k_{1}, \ldots, k_{r_{1}}\right) x_{0} .
\end{aligned}
$$

To make contact with the discussion below equation (3.12), suppose that $r_{\ell} \in\{1,2\}$ and all operators $\hat{a}_{\mathrm{ns}}^{(\ell)}$ of a given order are equal. For $r_{\ell}=1$ we set $\hat{a}_{\mathrm{ns}}:=\hat{a}_{\mathrm{ns}}^{(\ell)}$ and for $r_{\ell}=2$ we write $\hat{b}_{\mathrm{ns}}:=\hat{a}_{\mathrm{ns}}^{(\ell)}$. Let us now define $X_{\mathrm{ns}, i}:=L^{2}\left(\mathbb{R}^{3}\right)^{\otimes i} \otimes \mathcal{H}$ so that $X_{\mathrm{ns}}=\bigoplus_{i \in \mathbb{N}_{0}} X_{\mathrm{ns}, i}$ and write $\hat{a}_{\mathrm{ns}, i}:=\hat{a} \uparrow X_{\mathrm{ns}, i-1}$ and $\hat{b}_{\mathrm{ns}, i+1, i}:=\hat{b}_{\mathrm{ns}} \uparrow X_{\mathrm{ns}, i-1}$. Clearly $\hat{a}_{\mathrm{ns}, i}: X_{\mathrm{ns}, i-1} \rightarrow X_{\mathrm{ns}, i}$ and $\hat{b}_{\mathrm{ns}, i+1, i}: X_{\mathrm{ns}, i-1} \rightarrow X_{\mathrm{ns}, i+1}$. More explicitly, we have

$$
\begin{aligned}
& \left(\hat{a}_{\mathrm{ns}, i} x_{\mathrm{ns}, i-1}\right)\left(k_{1}, \ldots, k_{i-1}, k_{i}\right)=\hat{a}_{\mathrm{ns}, i}\left(k_{1}, \ldots, k_{i-1}, k_{i}\right) x_{\mathrm{ns}, i-1}\left(k_{1}, \ldots, k_{i-1}\right) \\
& \left(\hat{b}_{\mathrm{ns}, i+1, i} x_{\mathrm{ns}, i-1}\right)\left(k_{1}, \ldots, k_{i-1}, k_{i}, k_{i+1}\right)=\hat{b}_{\mathrm{ns}, i+1, i}\left(k_{1}, \ldots, k_{i-1}, k_{i}, k_{i+1}\right) x_{\mathrm{ns}, i-1}\left(k_{1}, \ldots, k_{i-1}\right)
\end{aligned}
$$

for $x_{\mathrm{ns}, i-1} \in X_{\mathrm{ns}, i-1}$. With the notation of Subsection 3.1 we can rewrite (3.24) as

$$
\begin{aligned}
\hat{a}_{\mathrm{ns}}^{(f)} \ldots \hat{a}_{\mathrm{ns}}^{(1)} x_{0} & =\left(\hat{a}_{\mathrm{ns}, m} \ldots \hat{b}_{\mathrm{ns}, i+1, i} \ldots \hat{b}_{\mathrm{ns}, i^{\prime}+1, i^{\prime}} \ldots \hat{a}_{\mathrm{ns}, 1}\right) x_{0} \\
& =\operatorname{Op}_{\hat{a}_{\mathrm{ns}}, \hat{b}_{\mathrm{ns}}}^{(m)}\left[a_{m} \ldots b_{i+1, i} \ldots b_{i^{\prime}+1, i^{\prime}} \ldots a_{1}\right] x_{0} \\
& =\hat{\delta}_{i} \ldots \hat{\delta}_{i^{\prime}} \mathrm{Op}_{\hat{a}_{\mathrm{ns}}, \hat{b}_{\mathrm{ns}}}^{(m)}\left[a_{m} \ldots a_{1}\right] x_{0},
\end{aligned}
$$

where $\hat{\boldsymbol{a}}_{\mathbf{n s}}:=\left\{\hat{a}_{i}\right\}_{i \in \mathbb{N}}$ and $\hat{\boldsymbol{b}}_{\mathbf{n s}}:=\left\{\hat{b}_{i+1, i}\right\}_{i \in \mathbb{N}}$.

Now let $\hat{a}=P_{\text {sym }} \hat{a}_{\mathrm{ns}}, \hat{b}=P_{\mathrm{sym}} \hat{b}_{\mathrm{ns}}$ and consider the non-commutative recurrence relation

$$
x_{n}=\hat{a} x_{n-1}+\hat{b} x_{n-2} .
$$

We have by formulas (3.12), (3.23), (3.27)

$$
\begin{aligned}
x_{n} & =\sum_{\ell=0}^{[n / 2]} \sum_{1 \leq i_{1} \ll \cdots \ll i_{\ell} \leq n-1} \operatorname{Op}_{\hat{a}, \hat{b}}^{(n)}\left[\delta_{i_{1}} \ldots \delta_{i_{\ell}} a_{n} \ldots a_{1}\right] x_{0} \\
& =P_{\mathrm{sym}} \sum_{\ell=0}^{[n / 2]} \sum_{1 \leq i_{1} \ll \cdots \ll i_{\ell} \leq n-1} \operatorname{Op}_{\hat{a}_{\mathrm{n},}, \hat{b}_{\mathrm{ns}}}^{(n)}\left[\delta_{i_{1}} \ldots \delta_{i_{\ell}} a_{n} \ldots a_{1}\right] x_{0} \\
& =P_{\mathrm{sym}} \sum_{\ell=0}^{[n / 2]} \sum_{1 \leq i_{1} \ll \cdots \ll i_{\ell} \leq n-1} \hat{\delta}_{i_{1}} \ldots \hat{\delta}_{i_{\ell}} \mathrm{Op}_{\hat{a}_{\mathrm{ns}}, \hat{b}_{\mathrm{ns}}}^{(n)}\left[a_{n} \ldots a_{1}\right] x_{0} \\
& =P_{\mathrm{sym}} \sum_{\ell=0}^{[n / 2]} \sum_{1 \leq i_{1} \ll \cdots \ll i_{\ell} \leq n-1}\left(\hat{b}_{\mathrm{ns}, i_{1}+1, i_{1}} \hat{\partial}_{i_{1}+1} \hat{\partial}_{i_{1}}\right) \ldots\left(\hat{b}_{\mathrm{ns}, i_{\ell}+1, i_{\ell}} \hat{\partial}_{i_{\ell}+1} \hat{\partial}_{i_{\ell}}\right) \mathrm{Op}_{\hat{a}_{\mathrm{ns}}, \hat{b}_{\mathrm{ns}}}^{(n)}\left[a_{n} \ldots a_{1}\right] x_{0},
\end{aligned}
$$


where in the last step we use the notation introduced below (3.5).

As anticipated at the beginning of Section 3.2, the solution $x_{n}$ - that we have constructed in a slightly general setting concerning the sequences of operator valued functions $\hat{a}_{\mathrm{ns}}=\left\{\hat{a}_{\mathrm{ns}, m}\right\}_{m \geq r}$ - will be applied to the recursive relation (4.51) for the vectors $\hat{f}_{P, \sigma}^{n}\left(k_{1}, \ldots, k_{n}\right)$. For this purpose, now let us consider the case of $\hat{a}_{\mathrm{ns}, i}, \hat{b}_{\mathrm{ns}, i+1, i}$ of the following form:

$$
\begin{aligned}
& \hat{a}_{\mathrm{ns}, i}\left(k_{1}, \ldots, k_{i-1}, k_{i}\right)=\sum_{j=1}^{3} g^{(j)}\left(k_{i}\right) \check{\mathrm{a}}_{\mathrm{ns}, i}^{(j)}\left(k_{1}, \ldots, k_{i-1}, k_{i}\right), \\
& \hat{b}_{\mathrm{ns}, i+1, i}\left(k_{1}, \ldots, k_{i-1}, k_{i}, k_{i+1}\right)=\left(\sum_{j=1}^{3} g^{(j)}\left(k_{i}\right) g^{(j)}\left(k_{i+1}\right)\right) \check{\mathrm{b}}_{\mathrm{ns}, i+1, i}\left(k_{1}, \ldots, k_{i-1}, k_{i}, k_{i+1}\right),
\end{aligned}
$$

where $\check{\mathrm{a}}_{\mathrm{ns}, i}^{(j)}, \check{\mathrm{b}}_{\mathrm{ns}, i+1, i}$ are $B(\mathcal{H})$-valued functions as specified above (3.18) and $g^{(j)}$ are complex valued, bounded measurable functions on $\mathbb{R}^{3}$. By substituting this to formula (3.29), we get

$$
\begin{aligned}
x_{n}= & P_{\mathrm{sym}} \sum_{\ell=0}^{[n / 2]} \sum_{1 \leq i_{1} \ll \ldots \ll i_{\ell} \leq n-1} \sum_{j_{1}, \ldots, j_{n}} g_{1}^{\left(j_{1}\right)} \ldots g_{n}^{\left(j_{n}\right)} \times \\
& \times\left(\check{\mathrm{b}}_{\mathrm{ns}, i_{1}+1, i_{1}} \hat{\partial}_{i_{1}+1} \hat{\partial}_{i_{1}}\right) \ldots\left(\check{\mathrm{b}}_{\mathrm{ns}, i_{\ell}+1, i_{\ell}} \hat{\partial}_{i_{\ell}+1} \hat{\partial}_{i_{\ell}}\right) \mathrm{Op}_{\check{a}_{\mathrm{ns}}, \breve{\mathrm{b}}_{\mathrm{ns}}}^{(n)}\left[a_{n}^{\left(j_{n}\right)} \ldots a_{1}^{\left(j_{1}\right)}\right] x_{0},
\end{aligned}
$$

where $\left(g_{1}^{\left(j_{1}\right)} \ldots g_{n}^{\left(j_{n}\right)}\right)\left(k_{1}, \ldots, k_{n}\right):=g^{\left(j_{1}\right)}\left(k_{1}\right) \ldots g^{\left(j_{n}\right)}\left(k_{n}\right), \check{\boldsymbol{a}}_{\mathrm{ns}}:=\left\{\check{\mathrm{a}}_{\mathrm{ns}, i}^{\left(j_{i}\right)}\right\}_{i \in \mathbb{N}, j_{i}=1,2,3}, \check{\boldsymbol{b}}_{\mathrm{ns}}:=\left\{\check{\mathrm{b}}_{\mathrm{ns}, i+1, i}^{\left(j_{j}\right)}\right\}_{i \in \mathbb{N}, j_{i}=1,2,3}$. As a slight generalization of our notations, the action of $\check{\mathrm{b}}_{\mathrm{ns}, i+1, i} \hat{\partial}_{i+1} \hat{\partial}_{i}$ is meant to replace $\check{\mathrm{a}}_{\mathrm{ns}, i+1}^{\left(j_{i+1}\right)} \breve{\mathrm{a}}_{\mathrm{ns}, i}^{\left(j_{j}\right)}$ with $\check{\mathrm{b}}_{\mathrm{ns}, i+1, i} \delta_{j_{i+1}, j_{i}}$, (where $\delta_{j_{i+1}, j_{i}}$ is the Kronecker delta, not to be confused with (3.1)), and thus recovers the scalar product $\sum_{j=1}^{3} g^{(j)}\left(k_{i}\right) g^{(j)}\left(k_{i+1}\right)$ from (3.31). In applications we will not write the summation over $j_{i}$ explicitly in our notation, since it has no influence on our estimates.

\section{Ground state wave functions of the Nelson model}

\subsection{Pull through identities}

In Lemmas 4.1, 4.2 below we recall standard pull-through identities from [Fr73]. They are used here to establish new pull-through identities in Lemmas 4.3-4.5 below which are then exploited in Subsection 4.3 to derive a novel formula for wave functions, more suitable for the analysis of infrared properties.

We define $C^{\infty}\left(H_{P, \text { free }}\right):=\bigcap_{\ell \geq 0} D\left(H_{P \text {,free }}^{\ell}\right)$ and state the following lemma which is obtained by a standard computation.

Lemma 4.1. [Fr73] For $\psi \in C^{\infty}\left(H_{P, \text { free }}\right)$ we have in the sense of vector valued distributions in $\tilde{k}$

$$
b(\tilde{k}) H_{P-k, \sigma} \psi=\left(H_{P-k-\tilde{k}, \sigma}+|\tilde{k}|\right) b(\tilde{k}) \psi+v_{\bar{\alpha}}^{\sigma}(\tilde{k}) \psi .
$$

Now we introduce the notation

$$
H_{P, \sigma ; k_{1}, \ldots, k_{n}}:=H_{P-\left(k_{1}+\cdots+k_{n}\right), \sigma}+\left|k_{1}\right|+\cdots+\left|k_{n}\right|
$$

and note the following simple consequence of Lemma 4.1. 
Lemma 4.2. [Fr73] For $\psi \in C^{\infty}\left(H_{P, \text { free }}\right)$ we have in the sense of vector valued distributions in $k_{1}, \ldots, k_{n}$

$$
b\left(k_{n}\right) \ldots b\left(k_{1}\right) H_{P, \sigma} \psi=H_{P, \sigma ; k_{1}, \ldots, k_{n}} b\left(k_{n}\right) \ldots b\left(k_{1}\right) \psi+\sum_{i=1}^{n} v_{\bar{\alpha}}^{\sigma}\left(k_{i}\right) b\left(k_{n}\right) \ldots \check{i} \ldots b\left(k_{1}\right) \psi .
$$

Proof. For the reader's convenience we provide a proof. By Lemma 4.1, equality (4.3) holds for $n=1$. Suppose the equality holds for $n$. Then we can compute

$$
\begin{aligned}
b\left(k_{n+1}\right) b\left(k_{n}\right) \ldots b\left(k_{1}\right) H_{P, \sigma} \psi= & b\left(k_{n+1}\right) H_{P, \sigma ; k_{1}, \ldots, k_{n}} b\left(k_{n}\right) \ldots b\left(k_{1}\right) \psi \\
& +b\left(k_{n}\right) \sum_{i=1}^{n} v_{\bar{\alpha}}^{\sigma}\left(k_{i}\right) b\left(k_{n-1}\right) \ldots \check{i} \ldots b\left(k_{1}\right) \psi \\
= & H_{P, \sigma ; k_{1}, \ldots, k_{n+1}} b\left(k_{n+1}\right) b\left(k_{n-1}\right) \ldots b\left(k_{1}\right) \psi \\
& +\sum_{i=1}^{n+1} v_{\bar{\alpha}}^{\sigma}\left(k_{i}\right) b\left(k_{n+1}\right) \ldots \check{i} \ldots b\left(k_{1}\right) \psi,
\end{aligned}
$$

where we used Lemma 4.1 with $k=k_{1}+\cdots+k_{n}$ and smearing in $k_{1}, \ldots, k_{n+1}$ is understood.

Let us now proceed to new pull through estimates which have better infrared properties. For this purpose we define

$$
\begin{aligned}
& f_{P, \sigma}(k):=\frac{v_{\bar{\alpha}}^{\sigma}(k)}{|k| \alpha_{P, \sigma}(\hat{k})}, \text { where } \quad \alpha_{P, \sigma}(\hat{k}):=\left(1-\hat{k} \cdot \nabla E_{P, \sigma}\right), \quad \hat{k}:=k /|k| \\
& g_{P, \sigma}(k):=f_{P, \sigma}(k) k, \\
& b_{W}(k):=b(k)+f_{P, \sigma}(k), \\
& \Lambda_{P, \sigma}:=\nabla E_{P, \sigma}-\left(P-P_{\mathrm{f}}\right),
\end{aligned}
$$

and note in passing that $b_{W}(k)=W_{P, \sigma}^{*} b(k) W_{P, \sigma}$ for

$$
W_{P, \sigma}:=e^{b^{*}\left(f_{P, \sigma}\right)-b\left(f_{P, \sigma}\right)}
$$

We have the following facts:

Lemma 4.3. For $\psi \in C^{\infty}\left(H_{P, \text { free }}\right)$ we have in the sense of vector-valued distributions in $k, \tilde{k}$

$$
\begin{aligned}
b_{W}(\tilde{k}) H_{P-k, \sigma} \psi & =\left(H_{P-(\tilde{k}+k), \sigma}+|\tilde{k}|\right) b_{W}(\tilde{k}) \psi-g_{P, \sigma}(\tilde{k}) \cdot\left(\Lambda_{P, \sigma}+k+\frac{1}{2} \tilde{k}\right) \psi, \\
b_{W}(\tilde{k}) \Lambda_{P, \sigma} \psi & =\left(\Lambda_{P, \sigma}+\tilde{k}\right) b_{W}(\tilde{k}) \psi-g_{P, \sigma}(\tilde{k}) \psi .
\end{aligned}
$$

Proof. First, using the definition in (4.8), we note the following identity on $C^{\infty}\left(H_{P, \text { free }}\right)$ :

$$
H_{P-(k+\tilde{k}), \sigma}=H_{P-k, \sigma}+\left(\Lambda_{P, \sigma}+k+\frac{1}{2} \tilde{k}\right) \cdot \tilde{k}-\nabla E_{P, \sigma} \cdot \tilde{k} .
$$

Omitting $\psi$ and using (4.7), the 1.h.s. of (4.10) has the following form

$$
\text { 1.h.s. }(4.10)=b(\tilde{k}) H_{P-k, \sigma}+f_{P, \sigma}(\tilde{k}) H_{P-k, \sigma} \text {. }
$$


Now we rearrange the r.h.s. of (4.10) making use of (4.12) and of the fact that $f_{P, \sigma}(\tilde{k}) \tilde{k}=: g_{P, \sigma}(\tilde{k})$.

$$
\begin{aligned}
\text { r.h.s. (4.10) } & =\left(H_{P-(\tilde{k}+k), \sigma}+|\tilde{k}|\right)\left(b(\tilde{k})+f_{P, \sigma}(\tilde{k})\right)-g_{P, \sigma}(\tilde{k}) \cdot\left(\Lambda_{P, \sigma}+k+\frac{1}{2} \tilde{k}\right) \\
& =\left(H_{P-(\tilde{k}+k), \sigma}+|\tilde{k}|\right) b(\tilde{k})+\left(H_{P-(\tilde{k}+k), \sigma}+|\tilde{k}|\right) f_{P, \sigma}(\tilde{k})-g_{P, \sigma}(\tilde{k}) \cdot\left(\Lambda_{P, \sigma}+k+\frac{1}{2} \tilde{k}\right) \\
& =\left(H_{P-(\tilde{k}+k), \sigma}+|\tilde{k}|\right) b(\tilde{k})+\left(H_{P-k, \sigma}+\left(\Lambda_{P, \sigma}+k+\frac{1}{2} \tilde{k}\right) \cdot \tilde{k}-\nabla E_{P, \sigma} \cdot \tilde{k}+|\tilde{k}|\right) f_{P, \sigma}(\tilde{k}) \\
& =\left(H_{P-(\tilde{k}+k), \sigma}+|\tilde{k}|\right) b(\tilde{k})+\left(H_{P-k, \sigma}-\nabla E_{P, \sigma} \cdot \tilde{k}+|\tilde{k}|\right) f_{P, \sigma}(\tilde{k}) .
\end{aligned}
$$

Now it is easy to see, making use of $\left(|\tilde{k}|-\nabla E_{P, \sigma} \cdot \tilde{k}\right) f_{P, \sigma}(\tilde{k})=v_{\bar{\alpha}}^{\sigma}(\tilde{k})$, that the equality between (4.13) and (4.14) is a consequence of Lemma 4.1.

Now we show (4.11). Its 1.h.s. has the form (omitting the vector $\psi$ )

$$
\text { 1.h.s. }(4.11)=\left(b(k)+f_{P, \sigma}(k)\right) \Lambda_{P, \sigma}=\left(b(k)+f_{P, \sigma}(k)\right)\left(\nabla E_{P, \sigma}-P+P_{\mathrm{f}}\right) \text {. }
$$

The r.h.s. is given by

$$
\begin{aligned}
\text { r.h.s. (4.11) } & =\left(\nabla E_{P, \sigma}-P+P_{\mathrm{f}}+\tilde{k}\right)\left(b(\tilde{k})+f_{P, \sigma}(\tilde{k})\right)-g_{P, \sigma}(\tilde{k}) \\
& =\left(\nabla E_{P, \sigma}-P+P_{\mathrm{f}}\right)\left(b(\tilde{k})+f_{P, \sigma}(\tilde{k})\right)+\tilde{k} b(\tilde{k}),
\end{aligned}
$$

where we made use again of the fact that $f_{P, \sigma}(\tilde{k}) \tilde{k}=: g_{P, \sigma}(\tilde{k})$. Now the equality of (4.15) and (4.16) follows from $b(\tilde{k}) P_{\mathrm{f}}=\left(P_{\mathrm{f}}+\tilde{k}\right) b(\tilde{k})$.

In the next lemma we will use the following definitions:

$$
\begin{aligned}
& G_{P, \sigma}\left(k^{\prime}, \tilde{k}\right):=g_{P, \sigma}\left(k^{\prime}\right) \cdot g_{P, \sigma}(\tilde{k}), \\
& F_{P, \sigma}(\tilde{k}, k):=-g_{P, \sigma}(\tilde{k}) \cdot\left(\Lambda_{P, \sigma}+k+\frac{1}{2} \tilde{k}\right) .
\end{aligned}
$$

In these terms, we immediately get from Lemma 4.3:

Lemma 4.4. For $\psi \in C^{\infty}\left(H_{P, \text { free }}\right)$ we have in the sense of vector-valued distributions in $\tilde{k}, k_{1}, \ldots, k_{n}$

$$
\begin{aligned}
& b_{W}(\tilde{k}) H_{P, \sigma ; k_{1}, \ldots, k_{n}} \psi=H_{P, \sigma ; \tilde{k}, k_{1}, \ldots, k_{n}} b_{W}(\tilde{k}) \psi+F_{P, \sigma}\left(\tilde{k}, k_{1}+\cdots+k_{n}\right) \psi, \\
& b_{W}(\tilde{k}) F_{P, \sigma}\left(k^{\prime}, k\right) \psi=F_{P, \sigma}\left(k^{\prime}, \tilde{k}+k\right) b_{W}(\tilde{k}) \psi+G_{P, \sigma}\left(k^{\prime}, \tilde{k}\right) \psi,
\end{aligned}
$$

where $H_{P, \sigma ; k_{1}, \ldots, k_{n}}$ is defined in (4.2).

Proof. Equality (4.19) is obtained from (4.10) by setting $k=k_{1}+\cdots+k_{n}$. As for (4.20) we have

$$
\begin{aligned}
b_{W}(\tilde{k}) F_{P, \sigma}\left(k^{\prime}, k\right) & =b_{W}(\tilde{k})(-) g_{P, \sigma}\left(k^{\prime}\right) \cdot\left(\Lambda_{P, \sigma}+k+\frac{1}{2} k^{\prime}\right) \\
& =(-) g_{P, \sigma}\left(k^{\prime}\right) \cdot\left(\left(\Lambda_{P, \sigma}+\tilde{k}+k+\frac{1}{2} k^{\prime}\right) b_{W}(\tilde{k})-g_{P, \sigma}(\tilde{k})\right) \\
& =F_{P, \sigma}\left(k^{\prime}, \tilde{k}+k\right) b_{W}(\tilde{k})+G_{P, \sigma}\left(k^{\prime}, \tilde{k}\right),
\end{aligned}
$$

where we made use of (4.11).

Now we can prove the n-particle pull-through identity: 
Lemma 4.5. For $\psi \in C^{\infty}\left(H_{P, \text { free }}\right)$ we have in the sense of vector-valued distributions in $k_{1}, \ldots, k_{n}$

$$
\begin{aligned}
b_{W}\left(k_{n}\right) \ldots b_{W}\left(k_{1}\right) H_{P, \sigma} \psi= & H_{P, \sigma ; k_{n}, \ldots, k_{1}} b_{W}\left(k_{n}\right) \ldots b_{W}\left(k_{1}\right) \psi \\
& +\sum_{i=1}^{n} F_{P, \sigma}\left(k_{i}, k_{1}+\cdots \check{i} \cdots+k_{n}\right) b_{W}\left(k_{n}\right) \cdots \check{i} \cdots b_{W}\left(k_{1}\right) \psi \\
& +\sum_{1 \leq i<i^{\prime} \leq n} G_{P, \sigma}\left(k_{i}, k_{i^{\prime}}\right) b_{W}\left(k_{n}\right) \cdots \check{i^{\prime}} \cdots \check{i} \cdots b_{W}\left(k_{1}\right) \psi .
\end{aligned}
$$

$\left(\right.$ For $n=1$ the $\sum_{i<i^{\prime}}$ part should be dropped and $\left.k_{1}+\cdots \check{i} \cdots+k_{n}=0\right)$.

Proof. We proceed by induction: For $n=1$ the statement follows from (4.19). Suppose the equality holds for $n$. Then

$$
\begin{aligned}
b_{W}\left(k_{n+1}\right) b_{W}\left(k_{n}\right) & \ldots b_{W}\left(k_{1}\right) H_{P, \sigma}=b_{W}\left(k_{n+1}\right) H_{P, \sigma ; k_{n}, \ldots, k_{1}} b_{W}\left(k_{n}\right) \ldots b_{W}\left(k_{1}\right) \\
& +\sum_{i=1}^{n} b_{W}\left(k_{n+1}\right) F_{P, \sigma}\left(k_{i}, k_{1}+\cdots \check{i} \cdots+k_{n}\right) b_{W}\left(k_{n}\right) \cdots \check{i} \cdots b_{W}\left(k_{1}\right) \\
& +\sum_{1 \leq i<i^{\prime} \leq n} G_{P, \sigma}\left(k_{i}, k_{i^{\prime}}\right) b_{W}\left(k_{n+1}\right) b_{W}\left(k_{n}\right) \cdots \check{i^{\prime}} \cdots \check{i} \cdots b_{W}\left(k_{1}\right) .
\end{aligned}
$$

Now we obtain by (4.19), (4.20)

$$
\begin{aligned}
& \begin{aligned}
(4.23)= & H_{P, \sigma ; k_{n+1}, k_{n}, \ldots, k_{1}} b_{W}\left(k_{n+1}\right) b_{W}\left(k_{n}\right) \ldots b_{W}\left(k_{1}\right) \\
& +F_{P, \sigma}\left(k_{n+1}, k_{1}+\cdots+k_{n}\right) b_{W}\left(k_{n}\right) \ldots b_{W}\left(k_{1}\right),
\end{aligned} \\
& \begin{aligned}
&(4.24)=\sum_{i=1}^{n} F_{P, \sigma}\left(k_{i}, k_{1}+\cdots \check{i} \cdots+\right.\left.k_{n}+k_{n+1}\right) b_{W}\left(k_{n+1}\right) b_{W}\left(k_{n}\right) \cdots \check{i} \cdots b_{W}\left(k_{1}\right) \\
&+\sum_{i=1}^{n} G_{P, \sigma}\left(k_{i}, k_{n+1}\right) b_{W}\left(k_{n}\right) \cdots \check{i} \cdots b_{W}\left(k_{1}\right), \\
&(4.25)=\sum_{1 \leq i<i^{\prime} \leq n} G_{P, \sigma}\left(k_{i}, k_{i^{\prime}}\right) b_{W}\left(k_{n+1}\right) b_{W}\left(k_{n}\right) \cdots \check{i^{\prime}} \cdots \check{i} \cdots b_{W}\left(k_{1}\right) .
\end{aligned}
\end{aligned}
$$

It is clear that

$$
\begin{aligned}
& (4.27)+(4.28)=\sum_{i=1}^{n+1} F_{P, \sigma}\left(k_{i}, k_{1}+\cdots \check{i} \cdots+k_{n+1}\right) b_{W}\left(k_{n+1}\right) \cdots \check{i} \cdots b_{W}\left(k_{1}\right), \\
& (4.29)+(4.30)=\sum_{1 \leq i<i^{\prime} \leq n+1} G_{P, \sigma}\left(k_{i}, k_{i^{\prime}}\right) b_{W}\left(k_{n+1}\right) \cdots \check{i^{\prime}} \cdots \check{i} \cdots b_{W}\left(k_{1}\right),
\end{aligned}
$$

which concludes the proof.

\subsection{Standard formula for the ground state wave functions}

As a simple application of the formalism of non-commutative recurrence relations, we first rederive the standard formula for the ground state wave functions of the Nelson model from [Fr73]. 
First, we define the following vector valued distributions

$$
\check{f}_{P, \sigma}^{n}\left(k_{1}, \ldots, k_{n}\right):=b\left(k_{1}\right) \ldots b\left(k_{n}\right) \check{\psi}_{P, \sigma}
$$

To analyse (4.33), we define for $P \in S$ and $k_{1}, \ldots, k_{n} \in \mathbb{R}^{3} \backslash\{0\}$

$$
\begin{aligned}
& H_{P, \sigma ; k_{1}, \ldots, k_{n}}:=H_{P-\left(k_{1}+\cdots+k_{n}\right), \sigma}+\left|k_{1}\right|+\cdots+\left|k_{n}\right|, \\
& R_{P, \sigma ; k_{1}, \ldots, k_{n}}:=\frac{1}{H_{P, \sigma ; k_{1}, \ldots, k_{n}}-E_{P, \sigma}}
\end{aligned}
$$

where the resolvent is well defined by Lemma 6.1 below. The following proposition is a consequence of of (4.3). We refer to Lemma D.3 of [DP17] for a detailed argument.

Proposition 4.6. $\check{f}_{P, \sigma}^{n}$ are elements of $L^{2}\left(\mathbb{R}^{3 n}\right) \otimes \Gamma\left(\mathfrak{h}_{\mathrm{fi}}\right)$ and satisfy the following recurrence relation for $n \geq 1$

$$
\check{f}_{P, \sigma}^{n}\left(k_{1}, \ldots, k_{n}\right)=(-) R_{P, \sigma ; k_{1}, \ldots, k_{n}} \sum_{i=1}^{n} v_{\bar{\alpha}}^{\sigma}\left(k_{i}\right) \check{f}_{P, \sigma}^{n-1}\left(k_{1}, \ldots \check{i} . ., k_{n}\right)
$$

with the initial condition $\check{f}_{P, \sigma}^{0}=\breve{\psi}_{P, \sigma}$.

We observe that formula (4.36) is a non-commutative recurrence relation on $X=\Gamma\left(L^{2}\left(\mathbb{R}^{3}\right)\right) \otimes$ $\Gamma\left(\mathfrak{h}_{\mathrm{fi}}\right)$ of the form (3.28) with $\hat{b}=0$. With the help of Lemma 3.3, we identify

$$
\begin{aligned}
& \hat{a}_{\mathrm{ns}, i}\left(k_{1}, \ldots, k_{i-1}, k_{i}\right)=i(-) R_{P, \sigma ; k_{1}, \ldots, k_{i}} v_{\bar{\alpha}}^{\sigma}\left(k_{i}\right), \\
& \hat{b}_{\mathrm{ns}, i+1, i}\left(k_{1}, \ldots, k_{i-1}, k_{i}, k_{i+1}\right)=0 .
\end{aligned}
$$

Setting $R_{P, \sigma ; i}\left(k_{1}, \ldots, k_{i}\right):=R_{P, \sigma ; k_{1}, \ldots, k_{i}}$, we get from formula (3.29)

$$
\check{f}_{P, \sigma}^{n}=n ! P_{\mathrm{sym}} \mathrm{Op}_{\hat{a}_{\mathrm{ns}}, \hat{b}_{\mathrm{ns}}}^{(n)}\left[a_{n} \ldots a_{1}\right] \breve{\psi}_{P, \sigma}=n !(-1)^{n} P_{\mathrm{sym}}\left(v_{\bar{\alpha} ; 1}^{\sigma} \ldots v_{\bar{\alpha} ; n}^{\sigma}\right)\left(R_{P, \sigma ; n} \ldots R_{P, \sigma ; 1}\right) \check{\psi}_{P, \sigma},
$$

where $\left(v_{\bar{\alpha} ; 1}^{\sigma} \ldots v_{\bar{\alpha} ; n}^{\sigma}\right)\left(k_{1}, \ldots, k_{n}\right):=v_{\bar{\alpha}}^{\sigma}\left(k_{1}\right) \ldots v_{\bar{\alpha}}^{\sigma}\left(k_{n}\right)$. Now consider the wave functions $\left\{f_{P, \sigma}^{n}\right\}_{n \in \mathbb{N}_{0}}$ of the ground state vector $\check{\psi}_{P, \sigma}$. Clearly,

$$
\begin{aligned}
f_{P, \sigma}^{n}\left(k_{1}, \ldots, k_{n}\right) & =\frac{1}{\sqrt{n !}}\left\langle\Omega, b\left(k_{1}\right) \ldots b\left(k_{n}\right) \check{\psi}_{P, \sigma}\right\rangle=\frac{1}{\sqrt{n !}}\left\langle\Omega, \check{f}_{P, \sigma}^{n}\left(k_{1}, \ldots, k_{n}\right)\right\rangle \\
& =\frac{1}{\sqrt{n !}} \sum_{\pi \in S_{n}}(-1)^{n}\left\langle\Omega, \prod_{i=n}^{1} \frac{1}{H_{P, \sigma ; k_{\pi(1)}, \ldots, k_{\pi(i)}}-E_{P, \sigma}} v_{\bar{\alpha}}^{\sigma}\left(k_{\pi(i)}\right) \check{\psi}_{P, \sigma}\right\rangle,
\end{aligned}
$$

where in the last step we used (4.39),(4.34). This is the standard formula for the wave functions of $\check{\psi}_{P, \sigma}$ from [Fr73]. 


\subsection{Novel formula for the ground state wave functions}

In this subsection we derive a novel formula for the ground state wave functions $f_{P, \sigma}^{n}$ which facilitates the study of infrared properties. For this purpose we recall that $b_{W}(k):=b(k)+f_{P, \sigma}(k)$, where $f_{P, \sigma}$ is given by (4.5), and set

$$
\hat{f}_{P, \sigma}^{n}\left(k_{1}, \ldots, k_{n}\right):=b_{W}\left(k_{1}\right) \ldots b_{W}\left(k_{n}\right) \check{\psi}_{P, \sigma},
$$

The $\Gamma\left(\mathfrak{h}_{\mathrm{fi}}\right)$-valued functions $\hat{f}_{P, \sigma}^{n}$ will be the main objects of our analysis. We know already from Proposition 4.6 that they are elements of $L^{2}\left(\mathbb{R}^{3 n}\right) \otimes \Gamma\left(\mathfrak{h}_{\mathrm{fi}}\right)$. They are related to the wave functions $f_{P, \sigma}^{n}$ as follows:

$$
\begin{aligned}
f_{P, \sigma}^{n}\left(k_{1}, \ldots, k_{n}\right) & =\frac{1}{\sqrt{n !}}\left\langle\Omega,\left(b_{W}\left(k_{1}\right)-f_{P, \sigma}\left(k_{1}\right)\right) \ldots\left(b_{W}\left(k_{n}\right)-f_{P, \sigma}\left(k_{n}\right)\right) \check{\psi}_{P, \sigma}\right\rangle \\
& =\frac{1}{\sqrt{n !}} \sum_{\ell=0}^{n}(-1)^{n-\ell} \sum_{\substack{1 \leq i_{1}<\ldots<i_{\ell} \leq n \\
1 \leq i_{\ell+1}<\ldots<i_{n} \leq n}}\left\langle\Omega, \hat{f}_{P, \sigma}^{n}\left(k_{i_{1}}, \ldots, k_{i_{\ell}}\right)\right\rangle f_{P, \sigma}\left(k_{i_{\ell+1}}\right) \ldots f_{P, \sigma}\left(k_{i_{n}}\right) .
\end{aligned}
$$

We have the following sufficient conditions for estimates (2.12).

Proposition 4.7. Suppose that

$$
\begin{aligned}
& \left\|\hat{f}_{P, \sigma}^{n}\left(k_{1}, \ldots, k_{n}\right)\right\| \leq g_{\sigma}^{n}\left(k_{1}, \ldots, k_{n}\right), \\
& \left\|\partial_{P^{j}} \hat{f}_{P, \sigma}^{n}\left(k_{1}, \ldots, k_{n}\right)\right\| \leq \sigma^{-\delta_{\lambda_{0}}} g_{\sigma}^{n}\left(k_{1}, \ldots, k_{n}\right), \\
& \left\|\partial_{P^{j}} \partial_{P^{j^{j}}} \hat{f}_{P, \sigma}^{n}\left(k_{1}, \ldots, k_{n}\right)\right\| \leq \sigma^{-\delta_{\lambda_{0}}} g_{\sigma}^{n}\left(k_{1}, \ldots, k_{n}\right) .
\end{aligned}
$$

Then $\sqrt{n !}\left|f_{P, \sigma}^{n}\left(k_{1}, \ldots, k_{n}\right)\right|, \sqrt{n !}\left|\partial_{P^{j}} f_{P, \sigma}^{n}\left(k_{1}, \ldots, k_{n}\right)\right|, \sqrt{n !}\left|\partial_{P^{j}} \partial_{P^{\prime}} f_{P, \sigma}^{n}\left(k_{1}, \ldots, k_{n}\right)\right|$ also satisfy the bounds (4.44), (4.45), (4.46), respectively.

Proof. This lemma follows immediately from formula (4.43), the bounds

$$
\left|f_{P, \sigma}(k)\right|,\left|\partial_{P^{j}} f_{P, \sigma}(k)\right| \leq c \frac{v_{\bar{\alpha}}^{\sigma}(k)}{|k|}, \quad\left|\partial_{P^{j}} \partial_{P^{j^{\prime}}} f_{P, \sigma}(k)\right| \leq c \sigma^{-\delta_{\lambda_{0}}} \frac{v_{\bar{\alpha}}^{\sigma}(k)}{|k|},
$$

which are a consequence of (2.6) and a simple combinatorial identity

$$
\sum_{\ell=0}^{n} \sum_{\substack{1 \leq i_{1}<\ldots<i_{\ell} \leq n \\
1 \leq i_{\ell+1}<\ldots<i_{n} \leq n}} 1=\sum_{\ell=0}^{n}\left(\begin{array}{l}
n \\
\ell
\end{array}\right)=2^{n}
$$

Let us now recall that $\Lambda_{P, \sigma}:=\nabla E_{P, \sigma}-\left(P-P_{\mathrm{f}}\right)$, introduce the following definitions

$$
\begin{aligned}
& g_{P, \sigma}(k):=f_{P, \sigma}(k) k, \\
& F_{P, \sigma}(\tilde{k}, k):=-g_{P, \sigma}(\tilde{k}) \cdot\left(\Lambda_{P, \sigma}+k+\frac{1}{2} \tilde{k}\right),
\end{aligned}
$$

and keep in mind definitions (4.34), (4.35). By standard energy bounds $R_{P, \sigma ; k_{1}, \ldots, k_{n}} \Lambda_{P, \sigma}$ are bounded operators on $\Gamma\left(\mathfrak{h}_{\mathrm{fi}}\right)$. Thus we can formulate the following proposition, which is a consequence of (4.22). We refer to Appendix B for a proof. 
Proposition 4.8. $\hat{f}_{P, \sigma}^{m}$ are elements of $L^{2}\left(\mathbb{R}^{3 m}\right) \otimes \Gamma\left(\mathfrak{h}_{\mathrm{fi}}\right)$ and satisfy the following recurrence relation for $n \geq 2$

$$
\begin{aligned}
\hat{f}_{P, \sigma}^{n}\left(k_{1}, \ldots, k_{n}\right)=(-) R_{P, \sigma ; k_{1}, \ldots k_{n}}( & \sum_{i=1}^{n} F_{P, \sigma}\left(k_{i}, k_{1}+\ldots \check{i} \ldots+k_{n}\right) \hat{f}_{P, \sigma}^{n-1}\left(k_{1}, \ldots \check{i} \ldots, k_{n}\right) \\
& \left.+\sum_{1 \leq i<i^{\prime} \leq n} g_{P, \sigma}\left(k_{i}\right) \cdot g_{P, \sigma}\left(k_{i^{\prime}}\right) \hat{f}_{P, \sigma}^{n-2}\left(k_{1}, \ldots \check{i} \ldots \check{\check{i}^{\prime}} \ldots, k_{n}\right)\right)
\end{aligned}
$$

with the initial conditions $\hat{f}_{P, \sigma}^{0}=\check{\psi}_{P, \sigma}, \hat{f}_{P, \sigma}^{1}(k)=(-) R_{P, \sigma ; k} F_{P, \sigma}(k, 0) \check{\psi}_{P, \sigma}$.

Equation (4.51) describes a non-commutative recurrence relation on $X_{\text {fin }} \subset X=\Gamma\left(L^{2}\left(\mathbb{R}^{3}\right)\right) \otimes$ $\Gamma\left(\mathfrak{h}_{\mathrm{fi}}\right)$ as discussed in Subsection 3.3. With the help of Lemma 3.3, we identify

$$
\begin{aligned}
\hat{a}_{\mathrm{ns}, i}\left(k_{1}, \ldots, k_{i-1}, k_{i}\right): & i g_{P, \sigma}\left(k_{i}\right) \cdot\left\{R_{P, \sigma ; k_{1}, \ldots, k_{i}}\left(\Lambda_{P, \sigma}+\left(k_{1}+\cdots+k_{i-1}\right)+\frac{1}{2} k_{i}\right)\right\} \\
& =i g_{P, \sigma}\left(k_{i}\right) \cdot\left\{R_{P, \sigma ; i}\left(\Lambda_{P, \sigma}+\underline{k}_{i-1}+\frac{1}{2} k_{i}\right)\right\}=i g_{P, \sigma}\left(k_{i}\right) \cdot\left\{R_{P, \sigma ; i} \Lambda_{P, \sigma ; i}\right\} \\
\hat{b}_{\mathrm{ns}, i+1, i}\left(k_{1}, \ldots, k_{i-1}, k_{i}, k_{i+1}\right): & =\frac{(i+1) i}{2}(-) g_{P, \sigma}\left(k_{i}\right) \cdot g_{P, \sigma}\left(k_{i+1}\right)\left\{R_{P, \sigma ; k_{1}, \ldots, k_{i+1}}\right\} \\
& =\frac{(i+1) i}{2}(-) g_{P, \sigma}\left(k_{i}\right) \cdot g_{P, \sigma}\left(k_{i+1}\right)\left\{R_{P, \sigma ; i+1}\right\},
\end{aligned}
$$

where we set $\underline{k}_{i}:=k_{1}+\cdots+k_{i}, \Lambda_{P, \sigma ; i}:=\Lambda_{P, \sigma}+\underline{k}_{i-1}+\frac{1}{2} k_{i}, R_{P, \sigma ; i}:=R_{P, \sigma ; k_{1}, \ldots, k_{i}}$ (defined in (4.35)) and call the expressions in curly brackets $\check{\mathrm{n}}_{\mathrm{ns}, i}:=R_{P, \sigma ; i} \Lambda_{P, \sigma ; i}, \check{\mathrm{b}}_{\mathrm{ns}, i+1, i}:=R_{P, \sigma ; i+1}$. Now formula (3.32) gives

$$
\begin{aligned}
\hat{f}_{P, \sigma}^{n}= & n ! P_{\mathrm{sym}} \sum_{\ell=0}^{[n / 2]} \sum_{2 \leq i_{1} \ll \cdots \ll i_{\ell} \leq n} \frac{(-1)^{\ell}}{2^{\ell}} g_{P, \sigma ; 1} \ldots g_{P, \sigma ; n} \times \\
& \times\left(R_{P, \sigma ; i_{1}} \hat{\partial}_{i_{1}} \hat{\partial}_{i_{1}-1}\right) \ldots\left(R_{P, \sigma ; i_{\ell}} \hat{\partial}_{i_{\ell}} \hat{\partial}_{i_{\ell}-1}\right) \mathrm{Op}_{\breve{a}_{\mathbf{n s}}, \breve{b}_{\mathbf{n s}}}^{(n)}\left[a_{n} \ldots a_{1}\right] \check{\psi}_{P, \sigma},
\end{aligned}
$$

where we skipped the summation over vector indices $j=1,2,3$ in the notation, shifted the indices $i_{i^{\prime}}$ and set $\left(g_{P, \sigma ; 1} \ldots g_{P, \sigma ; n}\right)\left(k_{1}, \ldots, k_{n}\right):=g_{P, \sigma}\left(k_{1}\right) \ldots g_{P, \sigma}\left(k_{n}\right)$. Relations (4.54), (4.43) constitute our novel formula for the ground state wave functions $f_{P, \sigma}^{n}$.

Since $\check{\boldsymbol{a}}_{\mathrm{ns}}, \check{\boldsymbol{b}}_{\mathrm{ns}}$ will not change in the future, we can simplify the notation further by writing:

$$
\alpha(n, \ldots, 1):=\mathrm{Op}_{\check{a}_{\mathrm{ns}}, \breve{b}_{\mathrm{ns}}}^{(n)}\left[a_{n} \ldots a_{1}\right] .
$$

Clearly, we have $\alpha(i)=R_{P, \sigma ; i} \Lambda_{P, \sigma ; i}$ and the following equality of operators holds:

$$
\alpha(n, \ldots, 1)=R_{P, \sigma ; n} \Lambda_{P, \sigma ; n} \ldots R_{P, \sigma ; 1} \Lambda_{P, \sigma ; 1} .
$$

However, we need to remember definition (4.55) in terms of the quantisation map to act on $\alpha(n, \ldots, 1)$ with $\check{\mathrm{b}}_{\mathrm{ns}, i_{\ell}+1, i_{\ell}} \hat{\partial}_{i_{\ell}+1} \hat{\partial}_{i_{\ell}}$. Thus the relevant expressions to study are

$$
I_{i_{1}, \ldots, i_{\ell}}:=\left(R_{P, \sigma ; i_{1}+1} \hat{\partial}_{i_{1}+1} \hat{\partial}_{i_{1}}\right) \ldots\left(R_{P, \sigma ; i_{\ell}+1} \hat{\partial}_{i_{\ell}+1} \hat{\partial}_{i_{\ell}}\right) \alpha(n, \ldots, 1) \check{\psi}_{P, \sigma}
$$


Let us now set

$$
\begin{aligned}
C_{\ell} & :=\left(i_{1}, \ldots, i_{\ell}\right), \text { where } 2 \leq i_{1} \ll \ldots \ll i_{\ell} \leq n, \\
C^{2 \ell} & :=\left(i_{1}-1, i_{1}, \ldots, i_{\ell}-1, i_{\ell}\right), \\
\hat{\delta}_{C_{\ell}} & :=\left(R_{P, \sigma ; i_{1}} \hat{\partial}_{i_{1}} \hat{\partial}_{i_{1}-1}\right) \ldots\left(R_{P, \sigma ; i_{\ell}} \hat{\partial}_{i_{\ell}} \hat{\partial}_{i_{\ell}-1}\right), \\
I_{C_{\ell}} & :=I_{i_{1}, \ldots, i_{\ell}}=\hat{\delta}_{C_{\ell}} \alpha(n, \ldots, 1) \check{\psi}_{P, \sigma},
\end{aligned}
$$

so that formula (4.54) reads

$$
\hat{f}_{P, \sigma}^{n}=n ! P_{\mathrm{sym}} \sum_{\ell=0}^{[n / 2]} \frac{(-1)^{\ell}}{2^{\ell}} \sum_{C_{\ell}} g_{C_{\ell}} I_{C_{\ell}}=n ! P_{\mathrm{sym}} \sum_{\ell=0}^{[n / 2]} \frac{(-1)^{\ell}}{2^{\ell}} \sum_{C_{\ell}} g_{C_{\ell}} \hat{\delta}_{C_{\ell}} \alpha(n, \ldots, 1) \check{\psi}_{P, \sigma},
$$

where $g_{C_{\ell}}\left(k_{1}, \ldots, k_{n}\right):=g_{P, \sigma}\left(k_{1}\right) \ldots g_{P, \sigma}\left(k_{n}\right)$ with the contraction pattern of indices $j_{i}$ dictated by $C_{\ell}$. The reader should keep in mind that $g_{C_{\ell}}, I_{C_{\ell}}, \alpha(\ldots)$ depend on $P, \sigma$, although this is suppressed in the notation. For future reference we also introduce the decompositions

$$
\hat{\delta}_{C_{\ell}}=: \hat{\delta}_{C_{\ell}}^{\prime}\left(R_{P, \sigma ; i} \hat{\delta}_{i} \hat{\delta}_{i-1}\right), \quad \hat{\delta}_{C_{\ell}}=: \hat{\delta}_{C_{\ell}}^{\prime \prime}\left(R_{P, \sigma ; i} \hat{\delta}_{i} \hat{\delta}_{i-1}\right)\left(R_{P, \sigma ; i} \hat{\delta}_{\hat{i}} \hat{\delta}_{\hat{i}-1}\right),
$$

where $\hat{\delta}_{C_{\ell}}^{\prime}$ (resp. $\hat{\delta}_{C_{\ell}}^{\prime \prime}$ ) denotes $\hat{\delta}_{C_{\ell}}$ with one (resp. two) factors omitted as indicated in (4.63). Which factors are omitted will be clear from the context.

\section{Derivatives of $\hat{f}_{P, \sigma}^{n}$ w.r.t. $P$}

In Proposition 4.7 we proved the desired bounds on the ground state wave functions $f_{P, \sigma}^{n}\left(k_{1}, \ldots, k_{n}\right)$ assuming suitable estimates on the derivatives in $P$ up to degree 2 of $\hat{f}_{P, \sigma}^{n}\left(k_{1}, \ldots, k_{n}\right)$. In the present section, namely in Subsections 5.1-5.2, we compute and organize the different contributions to the derivatives in $P$ of $\hat{f}_{P, \sigma}^{n}\left(k_{1}, \ldots, k_{n}\right)$. In Proposition 5.1 we show that the assumptions upon which Proposition 4.7 is based follow from some estimates of the contributions computed in Subsections 5.1-5.2. The lengthy verification of the latter estimates (assumed in Proposition 5.1) is deferred to Section 6.

\subsection{First derivatives}

In order to analyse $\partial_{P} I_{C_{\ell}}$ it is convenient to introduce operators $\partial_{R_{i}}, \partial_{\Lambda_{i}}$ and $\partial_{\breve{\psi}}$, which act as $\partial_{P}$ only via the indicated expressions $\left(R_{i}, \Lambda_{i}\right.$, and $\breve{\psi}$, respectively). ${ }^{1}$ To define them, let us introduce for a moment a more explicit notation

$$
I_{C_{\ell}}=: I_{C_{\ell}}\left(R_{P, \sigma ; n}, \ldots, R_{P, \sigma ; 1} ; \Lambda_{P, \sigma ; n}, \ldots, \Lambda_{P, \sigma ; 1} ; \breve{\psi}_{P, \sigma}\right)
$$

Now we set

$$
\begin{aligned}
& \partial_{R_{i}} I_{C_{\ell}}:=\left.\partial_{\tilde{P}} I_{C_{\ell}}\left(R_{P, \sigma ; n}, \ldots, R_{\tilde{P}, \sigma ; i}, \ldots R_{P, \sigma ; 1} ; \Lambda_{P, \sigma ; n}, \ldots, \Lambda_{P, \sigma ; 1} ; \check{\psi}_{P, \sigma}\right)\right|_{\tilde{P}=P}, \\
& \partial_{\Lambda_{i}} I_{C_{\ell}}:=\left.\partial_{\tilde{P}} I_{C_{\ell}}\left(R_{P, \sigma ; n}, \ldots, R_{P, \sigma ; 1} ; \Lambda_{P, \sigma ; n}, \ldots, \Lambda_{\tilde{P}, \sigma ; i}, \ldots, \Lambda_{P, \sigma ; 1} ; \breve{\psi}_{P, \sigma}\right)\right|_{\tilde{P}=P}, \\
& \partial_{\tilde{\psi}} I_{C_{\ell}}:=\left.\partial_{\tilde{P}} I_{C_{\ell}}\left(R_{P, \sigma ; n}, \ldots, R_{P, \sigma, 1} ; \Lambda_{P, \sigma ; n}, \ldots, \Lambda_{P, \sigma ; 1} ; \check{\psi}_{\tilde{P}, \sigma}\right)\right|_{\tilde{P}=P},
\end{aligned}
$$

\footnotetext{
${ }^{1} \partial_{P}$ in the following denotes $\partial_{P^{j}}$ and $\partial_{P}^{2}$ denotes $\partial_{P^{j^{\prime}}} \partial_{P^{j}}$ for some $j, j^{\prime}=1,2,3$ which we do not write explicitly.
} 
so that obviously we have

$$
\partial_{P} I_{C_{\ell}}=\sum_{i=1}^{n}\left(\partial_{R_{i}} I_{C_{\ell}}+\partial_{\Lambda_{i}} I_{C_{\ell}}\right)+\partial_{\breve{\psi}} I_{C_{\ell}} .
$$

Let us now see in more detail how these derivatives act: For $i \notin C^{2 \ell}$ (defined in (4.59)) we have

$$
\begin{aligned}
\partial_{R_{i}} I_{C_{\ell}} & =\hat{\delta}_{C_{\ell}} \alpha(n, \ldots, i+1)\left(\partial_{P} R_{P, \sigma ; i}\right) \Lambda_{P, \sigma ; i} \alpha(i-1, \ldots, 1) \check{\psi}_{P, \sigma} \\
& =\hat{\delta}_{C_{\ell}} \alpha(n, \ldots, i+1) R_{P, \sigma ; i} \Lambda_{P, \sigma}^{i} R_{P, \sigma ; i} \Lambda_{P, \sigma ; i} \alpha(i-1, \ldots, 1) \check{\psi}_{P, \sigma} \\
& =\hat{\delta}_{C_{\ell}} \alpha(n, \ldots, i+1) R_{P, \sigma ; i} \Lambda_{P, \sigma}^{i} \alpha(i, \ldots, 1) \check{\psi}_{P, \sigma},
\end{aligned}
$$

where we made use of (6.12) and set $\Lambda_{P, \sigma}^{i}:=\Lambda_{P, \sigma}+\underline{k}_{i}$. Now suppose that $i \in C^{2 \ell}$. Then, we can assume that $i \in C_{\ell}$, since otherwise the resolvent $R_{P, \sigma ; i}$ does not appear in the expression and the result is zero. For $i \in C_{\ell}$ we have

$$
\begin{aligned}
\partial_{R_{i}} I_{C_{\ell}} & =\hat{\delta}_{C_{\ell}}^{\prime}\left(\left(\partial_{P} R_{P, \sigma ; i}\right) \hat{\delta}_{i} \hat{\delta}_{i-1}\right) \alpha(n, \ldots, 1) \check{\psi}_{P, \sigma} \\
& =\hat{\delta}_{C_{\ell}}^{\prime}\left(R_{P, \sigma ; i} \Lambda_{P, \sigma}^{i} R_{P, \sigma ; i} \hat{\delta}_{i} \hat{\delta}_{i-1}\right) \alpha(n, \ldots, 1) \check{\psi}_{P, \sigma} \\
& =\hat{\delta}_{C_{\ell}}^{\prime} \alpha(n, \ldots, i+1) R_{P, \sigma ; i} \Lambda_{P, \sigma}^{i} R_{P, \sigma ; i} \alpha(i-2, \ldots, 1) \check{\psi}_{P, \sigma} .
\end{aligned}
$$

Now we consider $\partial_{\Lambda_{i}}$. When computing the first derivative we can set $i \notin C^{2 \ell}$, since otherwise the expression does not contain $\Lambda_{P, \sigma ; i}$ and the result is zero. We have

$$
\begin{aligned}
\partial_{\Lambda_{i}} I_{C_{\ell}} & =\hat{\delta}_{C_{\ell}} \alpha(n, \ldots, i+1) R_{P, \sigma ; i}\left(\partial_{P} \Lambda_{P, \sigma ; i}\right) \alpha(i-1, \ldots, 1) \check{\psi}_{P, \sigma} \\
& =\left(\partial_{P} \Lambda_{P, \sigma ; i}\right) \hat{\delta}_{C_{\ell}} \alpha(n, \ldots, i+1) R_{P, \sigma ; i} \alpha(i-1, \ldots, 1) \check{\psi}_{P, \sigma},
\end{aligned}
$$

where we made use of the fact that $\partial_{P} \Lambda_{P, \sigma ; i}$ is a number. Finally $\partial_{\breve{\psi}}$ acts in an obvious manner

$$
\begin{aligned}
\partial_{\breve{\psi}} I_{C_{\ell}} & =\hat{\delta}_{C_{\ell}} \alpha(n, \ldots, 1) \partial_{P} \check{\psi}_{P, \sigma} \\
& =\hat{\delta}_{C_{\ell}} \alpha(n, \ldots, 1) R_{P, \sigma} \Lambda_{P, \sigma} \check{\psi}_{P, \sigma},
\end{aligned}
$$

where in the second step we used that $\partial_{P} \breve{\psi}_{P, \sigma}=R_{P, \sigma} \Lambda_{P, \sigma} \breve{\psi}, R_{P, \sigma}=\left(H_{P, \sigma}-E_{P, \sigma}\right)^{-1}$ (see formula (6.15)).

\subsection{Second derivatives}

Let us now proceed to $\partial_{P}^{2} I_{C_{\ell}}$. To analyse it, we will define the second derivatives $\partial_{R_{i^{\prime}}} \partial_{R_{i}} I_{C_{\ell}}$, $\partial_{\Lambda_{i^{\prime}}} \partial_{R_{i}} I_{C_{\ell}}, \partial_{\breve{\psi}} \partial_{R_{i}} I_{C_{\ell}}, \partial_{R_{i^{\prime}}} \partial_{\Lambda_{i}} I_{C_{\ell}}, \partial_{\Lambda_{i^{\prime}}} \partial_{\Lambda_{i}} I_{C_{\ell}}, \partial_{\breve{\psi}} \partial_{\Lambda_{i}} I_{C_{\ell}}, \partial_{\breve{\psi}}^{2} I_{C_{\ell}}$, by which we mean that the two derivatives $\partial_{P}$ act via the indicated expressions $R_{i}, \Lambda_{i}$, and $\breve{\psi}$ as we explain below formula (5.10.We supplement these definitions with

$$
\partial_{R_{i}} \partial_{\breve{\psi}} I_{C_{\ell}}:=\partial_{\breve{\psi}} \partial_{R_{i}} I_{C_{\ell}}, \quad \partial_{\Lambda_{i}} \partial_{\breve{\psi}} I_{C_{\ell}}:=\partial_{\breve{\psi}} \partial_{\Lambda_{i}} I_{C_{\ell}} .
$$

Informally speaking, the previous derivatives are defined by iterating the computational rules (5.6)(5.9) and taking the following specifications into account:

1. $\partial_{\Lambda_{i}}$ acts also on $\Lambda_{P, \sigma}^{i}$ appearing in (5.6) and (5.7). 
2. $\partial_{\Lambda_{i}}$ acts also on the numerical function $\left(\partial_{P} \Lambda_{P, \sigma ; i}\right)$ appearing in (5.8), changing it into $\left(\partial_{P}^{2} \Lambda_{P, \sigma ; i}\right)$.

3. $\partial_{\Lambda_{i}}$ ignores $\Lambda_{P, \sigma}$ appearing in (5.9), as this contribution is taken care of by the second application of $\partial_{\breve{\psi}}$.

Now we give formal definitions starting with the derivatives of $\partial_{R_{i}} I_{C_{\ell}}$ : Given formulas (5.6), (5.7) we can introduce for a moment an explicit notation

$$
\partial_{R_{i}} I_{C_{\ell}}=: \partial_{R_{i}} I_{C_{\ell}}\left(R_{P, \sigma ; n}, \ldots, R_{P, \sigma ; i+1}, R_{P, \sigma ; i} \Lambda_{P, \sigma}^{i}, R_{P, \sigma ; i}, \ldots R_{P, \sigma ; 1} ; \Lambda_{P, \sigma ; n}, \ldots, \Lambda_{P, \sigma ; 1} ; \breve{\psi}_{P, \sigma}\right)
$$

which is meaningful both for $i \notin C^{2 \ell}$ and $i \in C_{\ell}$. (In the latter case there is no dependence on $R_{P, \sigma ; i-1}$ and $\Lambda_{P, \sigma ; i-1}$, however). Now we define:

$\partial_{R_{i^{\prime}}} \partial_{R_{i}} I_{C_{\ell}}:=\left\{\begin{array}{l}\left.\partial_{\tilde{P}} \partial_{R_{i}} I_{C_{\ell}}\left(R_{P, \sigma ; n}, \ldots, R_{\tilde{P}, \sigma ; i^{\prime}}, \ldots, R_{P, \sigma ; i} \Lambda_{P, \sigma}^{i}, R_{P, \sigma ; i}, \ldots R_{P, \sigma ; 1} ; \Lambda_{P, \sigma ; n}, \ldots, \Lambda_{P, \sigma ; 1} ; \check{\psi}_{P, \sigma}\right)\right|_{\tilde{P}=P}, \\ \left.\partial_{\tilde{P}} \partial_{R_{i}} I_{C_{\ell}}\left(R_{P, \sigma ; n}, \ldots, R_{\tilde{P}, \sigma ; i} \Lambda_{P, \sigma}^{i}, R_{\tilde{P}, \sigma ; i}, \ldots R_{P, \sigma ; 1} ; \Lambda_{P, \sigma ; n}, \ldots, \Lambda_{P, \sigma ; 1} ; \check{\psi}_{P, \sigma}\right)\right|_{\tilde{P}=P},\end{array}\right.$

$\partial_{\Lambda_{i^{\prime}}} \partial_{R_{i}} I_{C_{\ell}}:=\left\{\begin{array}{l}\left.\partial_{\tilde{P}} \partial_{R_{i}} I_{C_{\ell}}\left(R_{P, \sigma ; n}, \ldots, R_{P, \sigma ; i} \Lambda_{P, \sigma}^{i}, R_{P, \sigma ; i}, \ldots, R_{P, \sigma ; 1} ; \Lambda_{P, \sigma ; n}, \ldots, \Lambda_{\tilde{P}, \sigma ; i^{\prime}}, \ldots, \Lambda_{P, \sigma ; 1} ; \breve{\psi}_{P, \sigma}\right)\right|_{\tilde{P}=P}, \\ \left.\partial_{\tilde{P}} \partial_{R_{i}} I_{C_{\ell}}\left(R_{P, \sigma ; n}, \ldots, R_{P, \sigma ; i} \Lambda_{\tilde{P}, \sigma}^{i}, R_{P, \sigma ; i}, \ldots, R_{P, \sigma ; 1} ; \Lambda_{P, \sigma ; n}, \ldots, \Lambda_{\tilde{P}, \sigma, i}, \ldots, \Lambda_{P, \sigma ; 1} ; \breve{\psi}_{P, \sigma}\right)\right|_{\tilde{P}=P},\end{array}\right.$

where the upper line in each of the two definitions holds for $i^{\prime} \neq i$ and the lower line for $i^{\prime}=i$. Furthermore, we set

$\partial_{\tilde{\psi}} \partial_{R_{i}} I_{C_{\ell}}:=\left.\partial_{\tilde{P}} \partial_{R_{i}} I_{C_{\ell}}\left(R_{P, \sigma ; n}, \ldots, R_{P, \sigma ; i} \Lambda_{P, \sigma}^{i}, R_{P, \sigma ; i}, \ldots, R_{P, \sigma ; 1} ; \Lambda_{P, \sigma ; n}, \ldots, \Lambda_{\tilde{P}, \sigma ; j}, \ldots, \Lambda_{P, \sigma ; 1} ; \check{\psi}_{\tilde{P}, \sigma}\right)\right|_{\tilde{P}=P}$

Next, we proceed to the derivatives of $\partial_{\Lambda_{i}} I_{C_{\ell}}$ for $i \notin C_{\ell}$. (We recall that $\partial_{\Lambda_{i}} I_{C_{\ell}}=0$ otherwise). Given formula (5.7) we can introduce for a moment an explicit notation:

$$
\partial_{\Lambda_{i}} I_{C_{\ell}}=: \partial_{\Lambda_{i}} I_{C_{\ell}}\left(R_{P, \sigma ; n}, \ldots, R_{P, \sigma ; 1} ; \Lambda_{P, \sigma ; n}, \ldots, \partial_{P} \Lambda_{P, \sigma ; i}, \ldots, \Lambda_{P, \sigma ; 1} ; \breve{\psi}_{P, \sigma}\right) .
$$

We define

$$
\begin{gathered}
\partial_{R_{i^{\prime}}} \partial_{\Lambda_{i}} I_{C_{\ell}}:=\left.\partial_{\tilde{P}} \partial_{\Lambda_{i}} I_{C_{\ell}}\left(R_{P, \sigma ; n}, \ldots, R_{\tilde{P}, \sigma ; i^{\prime}}, \ldots, R_{P, \sigma ; 1} ; \Lambda_{P, \sigma ; n}, \ldots, \partial_{P} \Lambda_{P, \sigma ; i}, \ldots, \Lambda_{P, \sigma ; 1} ; \check{\psi}_{P, \sigma}\right)\right|_{\tilde{P}=P},(5,1) \\
\partial_{\Lambda_{i^{\prime}}} \partial_{\Lambda_{i}} I_{C_{\ell}}:=\left\{\begin{array}{l}
\left.\partial_{\tilde{P}} \partial_{\Lambda_{i}} I_{C_{\ell}}\left(R_{P, \sigma ; n}, \ldots, R_{P, \sigma ; 1} ; \Lambda_{P, \sigma ; n}, \ldots, \Lambda_{\tilde{P}, \sigma ; i^{\prime}}, \ldots, \partial_{P} \Lambda_{P, \sigma ; i}, \ldots, \Lambda_{P, \sigma ; 1} ; \check{\psi}_{P, \sigma}\right)\right|_{\tilde{P}=P}, \\
\left.\partial_{\tilde{P}} \partial_{\Lambda_{i}} I_{C_{\ell}}\left(R_{P, \sigma ; n}, \ldots, R_{P, \sigma ; 1} ; \Lambda_{P, \sigma ; n}, \ldots, \partial_{\tilde{P}} \Lambda_{\tilde{P}, \sigma ; i}, \ldots, \Lambda_{P, \sigma ; 1} ; \breve{\psi}_{P, \sigma}\right)\right|_{\tilde{P}=P},
\end{array}\right.
\end{gathered}
$$

where the upper line holds for $i^{\prime} \neq i$ and the lower line for $i^{\prime}=i$. Also, we write

$$
\partial_{\check{\psi}} \partial_{\Lambda_{i}} I_{C_{\ell}}:=\left.\partial_{\tilde{P}} \partial_{\Lambda_{i}} I_{C_{\ell}}\left(R_{P, \sigma ; n}, \ldots, R_{P, \sigma ; 1} ; \Lambda_{P, \sigma ; n}, \ldots, \partial_{P} \Lambda_{P, \sigma ; i}, \ldots, \Lambda_{P, \sigma ; 1} ; \check{\psi}_{\tilde{P}, \sigma}\right)\right|_{\tilde{P}=P}
$$

Finally, given formula (5.8), we write

$$
\partial_{\breve{\psi}} I_{C_{\ell}}=: I_{C_{\ell}}\left(R_{P, \sigma ; n}, \ldots, R_{P, \sigma ; 1} ; \Lambda_{P, \sigma ; n}, \ldots, \Lambda_{P, \sigma ; 1} ; \partial_{P} \breve{\psi}_{P, \sigma}\right)
$$

and define in an obvious manner

$$
\partial_{\breve{\psi}}^{2} I_{C_{\ell}}:=\left.\partial_{\tilde{P}} \partial_{\breve{\psi}} I_{C_{\ell}}\left(R_{P, \sigma ; n}, \ldots, R_{P, \sigma ; 1} ; \Lambda_{P, \sigma ; n}, \ldots, \Lambda_{P, \sigma ; 1} ; \partial_{\tilde{P}} \check{\psi}_{\tilde{P}, \sigma}\right)\right|_{\tilde{P}=P}
$$


To conclude, we note that $\partial_{R_{i}} \partial_{\Lambda_{i^{\prime}}} I_{C_{\ell}}=\partial_{\Lambda_{i^{\prime}}} \partial_{R_{i}} I_{C_{\ell}}$ for $i \neq i^{\prime}$ but for $i=i^{\prime}$ this equality may fail due to specification 1. above. We also observe that $\partial_{R_{i}} \partial_{R_{i^{\prime}}} I_{C_{\ell}}=\partial_{R_{i^{\prime}}} \partial_{R_{i}} I_{C_{\ell}}$ and $\partial_{\Lambda_{i}} \partial_{\Lambda_{i^{\prime}}} I_{C_{\ell}}=\partial_{\Lambda_{i^{\prime}}} \partial_{\Lambda_{i}} I_{C_{\ell}}$ for all $i, i^{\prime}$ and recall (5.10).

By inspection of the definitions in this and the previous subsection we see that the appearances of $\tilde{P}$ cover all possible dependencies of $I_{C_{\ell}}$ on $P$. Therefore

$$
\begin{aligned}
\partial_{P}^{2} I_{C_{\ell}} & =\left(\sum_{i^{\prime}=1}^{n}\left(\partial_{R_{i^{\prime}}}+\partial_{\Lambda_{i^{\prime}}}\right)+\partial_{\breve{\psi}}\right)\left(\sum_{i=1}^{n}\left(\partial_{R_{i}}+\partial_{\Lambda_{i}}\right)+\partial_{\breve{\psi}}\right) I_{C_{\ell}} \\
& =\sum_{i, i^{\prime}=1}^{n}\left(\partial_{R_{i^{\prime}}}+\partial_{\Lambda_{i^{\prime}}}\right)\left(\partial_{R_{i}}+\partial_{\Lambda_{i}}\right) I_{C_{\ell}}+2 \sum_{i=1}^{n}\left(\partial_{R_{i}}+\partial_{\Lambda_{i}}\right) \partial_{\breve{\psi}} I_{C_{\ell}}+\partial_{\breve{\psi}}^{2} I_{C_{\ell}}
\end{aligned}
$$

\subsection{Sufficient condition for estimates (4.44), (4.45), (4.46)}

Proposition 5.1. Suppose that

$$
\begin{aligned}
& \left\|I_{C_{\ell}}\right\| \leq c^{n} \prod_{m^{\prime}=1}^{n}|\underline{k}|_{m^{\prime}}^{-1}, \\
& \left\|\partial_{R_{i}} I_{C_{\ell}}\right\|,\left\|\partial_{\Lambda_{i}} I_{C_{\ell}}\right\|,\left\|\partial_{\breve{\psi}} I_{C_{\ell}}\right\| \leq c^{n} \sigma^{-\delta_{\lambda_{0}}} \prod_{m^{\prime}=1}^{n}|\underline{k}|_{m^{\prime}}^{-1}, \\
& \left\|\partial_{R_{i^{\prime}}} \partial_{R_{i}} I_{C_{\ell}}\right\|,\left\|\partial_{R_{i^{\prime}}} \partial_{\Lambda_{i}} I_{C_{\ell}}\right\|,\left\|\partial_{R_{i^{\prime}}} \partial_{\breve{\psi}} I_{C_{\ell}}\right\| \leq c^{n} \sigma^{-\delta_{\lambda_{0}}} \prod_{m^{\prime}=1}^{n}|\underline{k}|_{m^{\prime}}^{-1}, \\
& \left\|\partial_{\Lambda_{i^{\prime}}} \partial_{\Lambda_{i}} I_{C_{\ell}}\right\|,\left\|\partial_{\Lambda_{i^{\prime}}} \partial_{R_{i}} I_{C_{\ell}}\right\|,\left\|\partial_{\Lambda_{i^{\prime}}} \partial_{\breve{\psi}} I_{C_{\ell}}\right\|,\left\|\partial_{\breve{\psi}}^{2} I_{C_{\ell}}\right\| \leq c^{n} \sigma^{-\delta_{\lambda_{0}}} \prod_{m^{\prime}=1}^{n}|\underline{k}|_{m^{\prime}}^{-1},
\end{aligned}
$$

where $|\underline{k}|_{m^{\prime}}:=\left|k_{1}\right|+\cdots+\left|k_{m^{\prime}}\right|$. Then the assumptions of Proposition 4.7 are satisfied, namely estimates (4.44), (4.45), (4.46) hold.

Proof. We collect several preparatory facts: First, we recall formula (4.62)

$$
\hat{f}_{P, \sigma}^{n}=n ! P_{\mathrm{sym}} \sum_{\ell=0}^{[n / 2]} \frac{(-1)^{\ell}}{2^{\ell}} \sum_{C_{\ell}} g_{C_{\ell}} I_{C_{\ell}}
$$

Next, since $g_{C_{\ell}}\left(k_{1}, \ldots, k_{n}\right):=g\left(k_{1}\right) \ldots g\left(k_{n}\right)$ and $g(k):=\left(v_{\bar{\alpha}}^{\sigma}(k) k\right) /\left(|k| \alpha_{P, \sigma}(\hat{k})\right)$ by definition (4.49) we have

$$
\begin{aligned}
& \left|g_{C_{\ell}}\left(k_{1}, \ldots, k_{n}\right)\right|,\left|\partial_{P} g_{C_{\ell}}\left(k_{1}, \ldots, k_{n}\right)\right| \leq c^{n} \prod_{j=1}^{n} v_{\bar{\alpha}}^{\sigma}\left(k_{j}\right), \\
& \left|\partial_{P}^{2} g_{C_{\ell}}\left(k_{1}, \ldots, k_{n}\right)\right| \leq c^{n} \sigma^{-\delta_{\lambda_{0}}} \prod_{j=1}^{n} v_{\bar{\alpha}}^{\sigma}\left(k_{j}\right),
\end{aligned}
$$

where we made use of (2.6). Moreover, we note that

$$
\sum_{\ell=0}^{[n / 2]} \frac{1}{2^{\ell}} \sum_{C_{\ell}} 1 \leq \sum_{\ell=0}^{n}\left(\begin{array}{l}
n \\
\ell
\end{array}\right)=2^{n}, \quad \sum_{\pi \in S_{n}} \prod_{m^{\prime}=1}^{n}|\underline{k}|_{\pi, m^{\prime}}^{-1}=\prod_{m^{\prime}=1}^{n}\left|k_{m^{\prime}}\right|^{-1},
$$


where $|\underline{k}|_{\pi, m^{\prime}}=\left|k_{\pi(1)}\right|+\cdots+\left|k_{\pi\left(m^{\prime}\right)}\right|$ and the latter equality in (5.25) is a combinatorial identity from [Fr73], which can be proven by induction.

From the information above and (5.18) we immediately obtain (4.44) because the $n$ ! in (5.22) is canceled by $\frac{1}{n !}$ from the symmetrization symbol $P_{\text {sym }}$ while the sum over the permutations is controlled by the identity on the left of (5.25). Let us now prove (4.45). We have

$$
\begin{aligned}
\partial_{P} \hat{f}_{P, \sigma}^{n}= & n ! P_{\mathrm{sym}} \sum_{\ell=0}^{[n / 2]} \frac{(-1)^{\ell}}{2^{\ell}} \sum_{C_{\ell}}\left(\partial_{P} g_{C_{\ell}}\right) I_{C_{\ell}} \\
& +n ! P_{\mathrm{sym}} \sum_{\ell=0}^{[n / 2]} \frac{(-1)^{\ell}}{2^{\ell}} \sum_{C_{\ell}} g_{C_{\ell}} \partial_{P} I_{C_{\ell}} .
\end{aligned}
$$

Term (5.26) is immediately estimated as before. We consider the second term:

$$
(5.27)=n ! P_{\mathrm{sym}} \sum_{\ell=0}^{[n / 2]} \frac{(-1)^{\ell}}{2^{\ell}} \sum_{C_{\ell}} g_{C_{\ell}}\left\{\sum_{i=1}^{n}\left(\partial_{R_{i}} I_{C_{\ell}}+\partial_{\Lambda_{i}} I_{C_{\ell}}\right)+\partial_{\breve{\psi}} I_{C_{\ell}}\right\}
$$

which is also easily estimated using (5.23), assumption (5.19) and the combinatorial relations (5.25). Finally, we analyse the second derivative. We have (schematically ${ }^{2}$ )

$$
\begin{aligned}
\partial_{P}^{2} \hat{f}_{P, \sigma}^{n}= & n ! P_{\mathrm{sym}} \sum_{\ell=0}^{[n / 2]} \frac{(-1)^{\ell}}{2^{\ell}}\left(\partial_{P}^{2} g_{C_{\ell}}\right) \sum_{C_{\ell}} I_{C_{\ell}} \\
& +n ! P_{\mathrm{sym}} \sum_{\ell=0}^{[n / 2]} \frac{(-1)^{\ell}}{2^{\ell}} \sum_{C_{\ell}} 2\left(\partial_{P} g_{C_{\ell}}\right) \partial_{P} I_{C_{\ell}} \\
& +n ! P_{\mathrm{sym}} \sum_{\ell=0}^{[n / 2]} \frac{(-1)^{\ell}}{2^{\ell}} \sum_{C_{\ell}} g_{C_{\ell}} \partial_{P}^{2} I_{C_{\ell}} .
\end{aligned}
$$

Terms (5.29) and (5.30) are estimated as before. To bound (5.31) we use formula (5.17) and assumptions (5.20), (5.21).

\section{Verification of assumptions of Proposition 5.1}

\subsection{Spectral ingredients}

In this subsection we collect spectral ingredients which constitute the key input needed to verify the assumptions of Proposition 5.1. We start with a list of relevant defnitions, some of which appeared before. For the sake of clarity in later parts of this section, we also limit the appearance

\footnotetext{
${ }^{2}$ Note that $2\left(\partial_{P} g_{C_{\ell}}\right) \partial_{P} I_{C_{\ell}}$ in $(5.30)$ actually means $\left(\partial_{P^{j}} g_{C_{\ell}}\right) \partial_{P^{\prime}} I_{C_{\ell}}+\left\{j \leftrightarrow j^{\prime}\right\}$.
} 
of the subscript $P, \sigma$ in our notation.

$$
\begin{aligned}
R & :=R_{P, \sigma}=\frac{1}{H_{P, \sigma}-E_{P, \sigma}}, \\
R_{i} & :=R_{P, \sigma ; i}:=\frac{1}{H_{P-\underline{k}_{i}, \sigma}-E_{P, \sigma}+|\underline{k}|_{i}}, \\
R^{i} & :=R_{P, \sigma}^{i}:=\frac{1}{H_{P, \sigma}-E_{P, \sigma}+r(\underline{k})_{i}}, \text { where } r(\underline{k})_{i}:=|\underline{k}|_{i}+\left|\underline{k}_{i}\right|^{2} / 2-\nabla E_{P, \sigma} \cdot \underline{k}_{i}, \\
\Lambda & :=\Lambda_{P, \sigma}=\nabla E_{P, \sigma}-\left(P-P_{\mathrm{f}}\right), \\
\Lambda_{i} & :=\Lambda+\underline{k}_{i-1}+\frac{1}{2} k_{i}, \\
\Lambda^{i} & :=\Lambda+\underline{k}_{i}, \\
\check{\psi} & :=\check{\psi}_{P, \sigma}, \\
Q & :=|\check{\psi}\rangle\langle\check{\psi}|,
\end{aligned}
$$

where $\underline{k}_{i}:=k_{1}+\cdots+k_{i}$ and $|\underline{k}|_{i}:=\left|k_{1}\right|+\cdots+\left|k_{i}\right|$. Of course $R$ is only defined on the range of $Q^{\perp}:=1-Q$. The following lemma treats the existence of the other resolvents.

Lemma 6.1. We have the following bounds

$$
\begin{aligned}
& \left\|R_{i}\right\|, \quad\left\|R_{i} \Lambda\right\|, \quad\left\|R_{i} Q^{\perp} \Lambda\right\| \leq \frac{c}{|\underline{k}|_{i}}, \\
& \|Q \Lambda\| \leq c .
\end{aligned}
$$

The same estimates hold if $R_{i}$ is replaced with $R^{i}$.

Proof. The estimate on $\left\|R_{i}\right\|$ uses formula (3.6) of [DP17]. $\left\|R_{i} \Lambda\right\|$ is easily reduced to estimating $\left\|R_{i}\left(P-P_{\mathrm{f}}-\underline{k}_{i}\right)^{2} R_{i}\right\|$ which can be handled by Lemma A.3 of [DP17]. To bound $\|Q \Lambda\|$ we write $Q \Lambda=\left(E_{P, \sigma}+i\right) Q\left(H_{P, \sigma}+i\right)^{-1} \Lambda$ and apply again Lemma A.3 of [DP17]. The estimate on $\left\|R_{i} Q^{\perp} \Lambda\right\|$ follows from the bounds on $\|Q \Lambda\|$ and $\left\|R_{i} \Lambda\right\|$. The bound on $\left\|R^{i}\right\|$ follows from the fact that $r(\underline{k})_{i} \geq$ $\frac{1}{2}|\underline{k}|_{i}$ which holds true due to $\left|\nabla E_{P, \sigma}\right| \leq 1 / 3+c|\lambda|$ (cf. formula (3.7) of [DP17]). Other bounds involving $R^{i}$ follow by similar considerations as above.

Furthermore, we note the following relations and estimates:

$$
\begin{aligned}
R_{i} & =R^{i}-R_{i}\left(\underline{k}_{i} \cdot \Lambda\right) R^{i}, \\
\partial_{P} R_{i} & =R_{i} \Lambda^{i} R_{i}, \\
\partial_{P} \Lambda & =O(1), \\
\partial_{P}^{2} \Lambda & =O\left(\sigma^{-\delta_{\lambda_{0}}}\right), \\
\partial_{P} \check{\psi} & =R \Lambda \check{\psi} .
\end{aligned}
$$

Here (6.11), (6.12) are obtained by straightforward computations, (6.13) and (6.14) follow from (2.6) and (6.15) was derived in Section 5 of [DP17].

Next we collect more sophisticated spectral estimates. The following theorem follows from the main technical results of [DP17] by maximal modulus principle arguments (explained in the same reference). 
Theorem 6.2. [DP17] Let $R(\Delta):=\left(H_{P, \sigma}-E_{P, \sigma}+\Delta\right)^{-1}$. Then

$$
\begin{aligned}
& \quad \sup _{\Delta \geq 0}\|R(\Delta) \Lambda \check{\psi}\| \leq c \sigma^{-\delta_{\lambda_{0}}}, \\
& \sup _{\Delta_{1}, \Delta_{2} \geq 0}\left\|R\left(\Delta_{1}\right) Q^{\perp} \Lambda R\left(\Delta_{2}\right) \Lambda \check{\psi}\right\| \leq c \sigma^{-\delta_{\lambda_{0}}} .
\end{aligned}
$$

Since $\Lambda \breve{\psi}$ is orthogonal to $\breve{\psi}$, so the 1.h.s. of (6.16), (6.17) are well defined also for $\Delta=\Delta_{2}=0$. Lemma 6.1 and Theorem 6.2 have the following corollary.

Corollary 6.3. With the above definitions, we have for $i \geq i^{\prime} \geq i^{\prime \prime}$

$$
\begin{aligned}
& \|R \Lambda \check{\psi}\| \leq c \sigma^{-\delta_{\lambda_{0}}}, \\
& \left\|R_{i} \Lambda_{i} \check{\psi}\right\| \leq c \sigma^{-\delta_{\lambda_{0}}}, \\
& \left\|R_{i} Q^{\perp} \Lambda_{i} R \Lambda \check{\psi}\right\| \leq c \sigma^{-\delta_{\lambda_{0}}}, \\
& \left\|R_{i} Q^{\perp} \Lambda_{i} R_{i^{\prime}} \Lambda_{i^{\prime}} \check{\psi}\right\| \leq c \sigma^{-\delta_{\lambda_{0}}}, \\
& \left\|R_{i} \Lambda_{i} R_{i^{\prime}} \check{\psi}\right\| \leq \sigma^{-\delta_{\lambda_{0}}} \frac{c}{|\underline{k}|_{i^{\prime}}}, \\
& \left\|R_{i} \Lambda_{i} R_{i^{\prime}} \Lambda_{i^{\prime}} R_{i^{\prime \prime}} \Lambda_{i^{\prime \prime}} \check{\psi}\right\| \leq \sigma^{-\delta_{\lambda_{0}}} \frac{c}{|\underline{k}|_{i^{\prime}}}, \\
& \left\|R_{i} \Lambda_{i} R_{i^{\prime}} \Lambda_{i^{\prime}} R \Lambda \check{\psi}\right\| \leq \sigma^{-\delta_{\lambda_{0}}} \frac{c}{|\underline{k}|_{i^{\prime}}} .
\end{aligned}
$$

The results remain true if some of $\Lambda_{i}$ are replaced with $\Lambda^{i}$ or $\Lambda$.

Proof. Property (6.18) follows directly from (6.16). To show (6.19) we shift the resolvent (see (6.11)), take (6.5) into account, and make use of (6.16) and (6.9):

$$
R_{i} \Lambda_{i} \breve{\psi}=R^{i} \Lambda_{i} \breve{\psi}-R_{i}\left(\underline{k}_{i} \cdot \Lambda\right) R^{i} \Lambda_{i} \breve{\psi}=O\left(\sigma^{-\delta_{\lambda_{0}}}\right) .
$$

In order to prove (6.20) we again make use of the shift of the resolvent, of the definition in (6.5), and of (6.17):

$$
R_{i} Q^{\perp} \Lambda_{i} R \Lambda \breve{\psi}=R^{i} Q^{\perp} \Lambda_{i} R \Lambda \check{\psi}-R_{i}\left(\underline{k}_{i} \cdot \Lambda\right) R^{i} Q^{\perp} \Lambda_{i} R \Lambda \check{\psi}=O\left(\sigma^{-\delta_{\lambda_{0}}}\right) .
$$

Concerning (6.21), we will repetitively use (6.17) and (6.19): We implement a resolvent shift

$$
\begin{aligned}
R_{i} Q^{\perp} \Lambda_{i} R_{i^{\prime}} \Lambda_{i^{\prime}} \breve{\psi}= & R_{i} Q^{\perp} \Lambda_{i} R^{i^{\prime}} \Lambda_{i^{\prime}} \check{\psi} \\
& -R_{i} Q^{\perp} \Lambda_{i} R_{i^{\prime}}\left(\underline{k}_{i^{\prime}} \cdot \Lambda\right) R^{i^{\prime}} \Lambda_{i^{\prime}} \check{\psi} .
\end{aligned}
$$

We consider the last two terms (6.27) and (6.28) separately. In the first one we implement a shift on the first resolvent from the left, and make use of the fact that $\Lambda_{i^{\prime}}=\Lambda+O\left(|\underline{k}|_{i^{\prime}}\right)$ :

$$
\begin{aligned}
(6.27)= & R^{i} Q^{\perp} \Lambda_{i} R^{i^{\prime}} \Lambda \check{\psi}-R_{i}\left(\underline{k}_{i} \cdot \Lambda\right) R^{i} Q^{\perp} \Lambda_{i} R^{i^{\prime}} \Lambda \check{\psi} \\
& +R^{i} Q^{\perp} \Lambda_{i} R^{i^{\prime}} O\left(|\underline{k}|_{i^{\prime}}\right) \check{\psi}-R_{i}\left(\underline{k}_{i} \cdot \Lambda\right) R^{i} Q^{\perp} \Lambda_{i} R^{i^{\prime}} O\left(|\underline{k}|_{i^{\prime}}\right) \check{\psi}=O\left(\sigma^{-\delta_{\lambda_{0}}}\right)
\end{aligned}
$$

With regard to (6.28) we split the identity operator in front of $R_{i^{\prime}}$ into $Q+Q^{\perp}$ :

$$
\begin{aligned}
(6.28)= & -R_{i} Q^{\perp} \Lambda_{i} R_{i^{\prime}} Q^{\perp}\left(\underline{k}_{i^{\prime}} \cdot \Lambda\right) R^{i^{\prime}} \Lambda_{i^{\prime}} \breve{\psi} \\
& -R_{i} Q^{\perp} \Lambda_{i} R_{i^{\prime}} Q\left(\underline{k}_{i^{\prime}} \cdot \Lambda\right) R^{i^{\prime}} \Lambda_{i^{\prime}} \check{\psi} .
\end{aligned}
$$


The last two terms give

$$
\begin{aligned}
(6.30)=- & \left(R_{i} \underline{k}_{i^{\prime}}\right) Q^{\perp} \Lambda_{i} R^{i^{\prime}} Q^{\perp} \Lambda R^{i^{\prime}} \Lambda_{i^{\prime}} \check{\psi} \\
& \left.+\left(R_{i} \underline{k}_{i^{\prime}}\right) Q^{\perp} \Lambda_{i} R_{i^{\prime}} \underline{k}_{i^{\prime}} \cdot \Lambda\right) R^{i^{\prime}} Q^{\perp} \Lambda R^{i^{\prime}} \Lambda_{i^{\prime}} \check{\psi}=O\left(\sigma^{-\delta_{\lambda_{0}}}\right), \\
(6.31)=- & R_{i} Q^{\perp} \Lambda_{i}\left(\underline{k}_{i^{\prime}} R^{i^{\prime}}\right) Q \Lambda R^{i^{\prime}} \Lambda_{i^{\prime}} \check{\psi} \\
& +\left(R_{i} \underline{k}_{i^{\prime}}\right) Q^{\perp} \Lambda_{i} R_{i^{\prime}}\left(\underline{k}_{i^{\prime}} \cdot \Lambda\right) R^{i^{\prime}} Q \Lambda R^{i^{\prime}} \Lambda_{i^{\prime}} \check{\psi}=O\left(\sigma^{-\delta_{\lambda_{0}}}\right),
\end{aligned}
$$

where we made use of (6.17) and (6.19) for (6.32) and (6.33), respectively.

Now we show (6.22): We shift the resolvent next to $\breve{\psi}$ and make use of (6.19):

$$
\begin{aligned}
R_{i} \Lambda_{i} R_{i^{\prime}} \breve{\psi} & \left.=R_{i} \Lambda_{i} R^{i^{\prime}} \breve{\psi}-R_{i} \Lambda_{i} R_{i^{\prime}} \underline{k}_{i^{\prime}} \cdot \Lambda\right) R^{i^{\prime}} \breve{\psi} \\
& =O\left(|\underline{k}|_{i^{\prime}}^{-1}\right) R_{i} \Lambda_{i} \check{\psi}-O\left(|\underline{k}|_{i^{\prime}}^{-1}\right)\left(\underline{k}_{i^{\prime}} R_{i}\right) \Lambda_{i} R_{i^{\prime}} \Lambda \breve{\psi}=O\left(|\underline{k}|_{i^{\prime}}^{-1} \sigma^{-\delta_{\lambda_{0}}}\right),
\end{aligned}
$$

where in the last step we made use of (6.19) and $\left(\underline{k}_{i^{\prime}} R_{i} \Lambda_{i}\right)=O(1)$.

To prove (6.23), we write

$$
R_{i} \Lambda_{i} R_{i^{\prime}} \Lambda_{i^{\prime}} R_{i^{\prime \prime}} \Lambda_{i^{\prime \prime}} \check{\psi}=R_{i} \Lambda_{i} R_{i^{\prime}} Q^{\perp} \Lambda_{i^{\prime}} R_{i^{\prime \prime}} \Lambda_{i^{\prime \prime}} \check{\psi}+R_{i} \Lambda_{i} R_{i^{\prime}} Q \Lambda_{i^{\prime}} R_{i^{\prime \prime}} \Lambda_{i^{\prime \prime}} \breve{\psi}
$$

apply (6.19), (6.21), (6.22) and the fact that $|\underline{k}|_{i}^{-1} \leq|\underline{k}|_{i^{\prime}}^{-1}$.

To verify (6.24) we proceed analogously. We write

$$
R_{i} \Lambda_{i} R_{i^{\prime}} \Lambda_{i^{\prime}} R \Lambda \check{\psi}=R_{i} \Lambda_{i} R_{i^{\prime}} Q^{\perp} \Lambda_{i^{\prime}} R \Lambda \check{\psi}+R_{i} \Lambda_{i} R_{i^{\prime}} Q \Lambda_{i^{\prime}} R \Lambda \check{\psi}
$$

apply (6.21), (6.22), (6.18) and the fact that $|\underline{k}|_{i}^{-1} \leq\left.\underline{\mid k}\right|_{i^{\prime}} ^{-1}$.

\subsection{Estimates on derivatives}

Proposition 6.4. There hold the bounds for $i \geq i^{\prime}$

$$
\begin{aligned}
\left\|I_{C_{\ell}}\right\| & \leq\left. c^{n} \prod_{m^{\prime}=1}^{n} \underline{\mid k}\right|_{m^{\prime}} ^{-1}, \\
\left\|\partial_{R_{i}} I_{C_{\ell}}\right\| & \leq c^{n} \sigma^{-\delta_{\lambda_{0}}} \prod_{m^{\prime}=1}^{n}|\underline{k}|_{m^{\prime}}^{-1}, \\
\left\|\partial_{R_{i^{\prime}}} \partial_{R_{i}} I_{C_{\ell}}\right\| & \leq c^{n} \sigma^{-\delta_{\lambda_{0}}} \prod_{m^{\prime}=1}^{n}|\underline{k}|_{m^{\prime}}^{-1} .
\end{aligned}
$$

Proof. Estimate (6.37) is obvious from formula (4.61), standard resolvent bounds (Lemma 6.1) and the fact that $\hat{\delta}_{C_{\ell}}$ can only decrease the number of resolvents. So we can proceed to the first derivative and divide the proof into several cases:

Case 1: $i \notin C^{2 \ell}$.

$$
\partial_{R_{i}} I_{C_{\ell}}=\hat{\delta}_{C_{\ell}} \partial_{R_{i}} \alpha(n, \ldots, 1) \check{\psi}=\hat{\delta}_{C_{\ell}} \alpha(n, \ldots, i+1) R_{i} \Lambda^{i} \alpha(i, \ldots, 1) \check{\psi}
$$


We consider several sub-cases.

- $1 \notin C^{2 \ell}$.

$$
\partial_{R_{i}} I_{C_{\ell}}=\hat{\delta}_{C_{\ell}} \alpha(n, \ldots, i+1) R_{i} \Lambda^{i} \alpha(i, \ldots, 2) R_{1} \Lambda_{1} \breve{\psi}=O\left(c^{n} \sigma^{-\delta_{\lambda_{0}}} \prod_{m^{\prime}=1}^{n}|\underline{k}|_{m^{\prime}}^{-1}\right),
$$

where we made use of standard resolvent bounds (Lemma 6.1), $R_{1} \Lambda_{1} \breve{\psi}=O\left(\sigma^{-\delta_{\lambda_{0}}}\right),|\underline{k}|_{i}^{-1} \leq|\underline{k}|_{1}^{-1}$ for $i \geq 1$ and the fact that the action of $\hat{\delta}_{C_{\ell}}$ can only decrease the number of resolvents.

$\bullet 1 \in C^{2 \ell}$.

$$
\begin{aligned}
\partial_{R_{i}} I_{C_{\ell}} & =\hat{\delta}_{C_{\ell}}^{\prime}\left(R_{2} \hat{\partial}_{2} \hat{\partial}_{1}\right) \alpha(n, \ldots, i+1) R_{i} \Lambda^{i} \alpha(i, \ldots, 3) R_{2} \Lambda_{2} R_{1} \Lambda_{1} \check{\psi} \\
& =\hat{\delta}_{C_{\ell}}^{\prime} \alpha(n, \ldots, i+1) R_{i} \Lambda^{i} \alpha(i, \ldots, 3) R_{2} \breve{\psi}=O\left(c^{n} \sigma^{-\delta_{\lambda_{0}}} \prod_{m^{\prime}=1}^{n}|\underline{k}|_{m^{\prime}}^{-1}\right),
\end{aligned}
$$

where we made use of Lemma $6.1,|\underline{k}|_{i}^{-1} \leq|\underline{k}|_{1}^{-1}$ for $i \geq 1$ and the fact that the action of $\hat{\delta}_{C_{\ell}}^{\prime}$ can only decrease the number of resolvents.

Case 2: $i \in C^{2 \ell}$. Clearly, for $i \in C^{2 \ell} \backslash C_{\ell}$ the $i$-th resolvent is eliminated from the expression by the action of $\left(R_{i+1} \hat{\partial}_{i+1} \hat{\partial}_{i}\right)$ so $\partial_{R_{i}} I_{C_{\ell}}=0$. Thus we can assume $i \in C_{\ell}$.

$$
\partial_{R_{i}} I_{C_{\ell}}=\hat{\delta}_{C_{\ell}}^{\prime}\left(R_{i} \Lambda^{i} R_{i} \hat{\partial}_{i} \hat{\partial}_{i-1}\right) \alpha(n, \ldots, 1) \check{\psi}=O\left(c^{n} \sigma^{-\delta_{\lambda_{0}}} \prod_{m^{\prime}=1}^{n}|\underline{k}|_{m^{\prime}}^{-1}\right),
$$

where we made use of Lemma 6.1 and $|\underline{k}|_{i}^{-1} \leq|\underline{k}|_{i-1}^{-1}$. This concludes the analysis of the first derivative and we proceed to the second derivative.

Case (1.1): $i, i^{\prime} \notin C^{2 \ell}$. Starting from formula (6.40), we have

$$
\partial_{R_{i^{\prime}}} \partial_{R_{i}} I_{C_{\ell}}=\hat{\delta}_{C_{\ell}} \alpha(n, \ldots, i+1) R_{i} \Lambda^{i} \alpha\left(i, \ldots, i^{\prime}+1\right) R_{i^{\prime}} \Lambda^{i^{\prime}} \alpha\left(i^{\prime}, \ldots, 1\right) \check{\psi},
$$

where $\alpha\left(i, \ldots, i^{\prime}+1\right)=1$ is understood for $i=i^{\prime}$. We consider several sub-cases here:

- $i^{\prime}=1, i \in\{1,2,3\}$. Consider the case ${ }^{3} i=1$ :

$$
\partial_{R_{i^{\prime}}} \partial_{R_{i}} I_{C_{\ell}}=\hat{\delta}_{C_{\ell}} \alpha(n, \ldots, 2) 2 R_{1} \Lambda^{1} R_{1} \Lambda^{1} R_{1} \Lambda_{1} \check{\psi}=O\left(c^{n} \sigma^{-\delta_{\lambda_{0}}} \prod_{m^{\prime}=1}^{n}|\underline{k}|_{m^{\prime}}^{-1}\right),
$$

where we made use of (6.23). The cases $i=2$ and $i=3$ can be treated analogously, making use of the fact that $|\underline{k}|_{2}^{-1},|\underline{k}|_{3}^{-1} \leq|\underline{k}|_{1}^{-1}$ and noting that for $i^{\prime}=1$ and $i \in\{1,2,3\}$ and $i, i^{\prime} \notin C^{2 \ell}$ we necessarily have $i_{1}-1>i$, so $\hat{\delta}_{C_{\ell}}$ does not interfere with the above argument.

- $i^{\prime}=1, i>3$.

$$
\partial_{R_{i^{\prime}}} \partial_{R_{i}} I_{C_{\ell}}=\hat{\delta}_{C_{\ell}} \alpha(n, \ldots, i+1) R_{i} \Lambda^{i} \alpha(i, \ldots, 2) R_{1} \Lambda^{1} R_{1} \Lambda_{1} \check{\psi}
$$

First, suppose $2 \notin C^{2 \ell}$. Then we can write $\alpha(i, \ldots, 2)=\alpha(i, \ldots, 3) R_{2} \Lambda_{2}$ apply (6.23) as in (6.45) and use $|\underline{k}|_{i}^{-1} \leq|\underline{k}|_{2}^{-1}$ to obtain the required bound. Next, suppose $2 \in C^{2 \ell}$. Then $\hat{\delta}_{C_{\ell}}$ eliminates $R_{2}$

\footnotetext{
${ }^{3}$ Expression (6.45) is somewhat schematic. $2 R_{1} \Lambda^{1} R_{1} \Lambda^{1} R_{1}$ stands for $R_{1}\left(\Lambda^{1}\right)^{j} R_{1}\left(\Lambda^{1}\right)^{j^{\prime}} R_{1}+\left\{j \leftrightarrow j^{\prime}\right\}$, but the indices $j, j^{\prime}=1,2,3$, coming from $\partial_{P^{j}} \partial_{P^{j^{\prime}}}$ are suppressed in our notation.
} 
from expression (6.46) so we can use $|\underline{k}|_{i}^{-1} \leq|\underline{k}|_{2}^{-1}$ and (6.19) to obtain a bound as in (6.45).

- $i^{\prime}=2, i \geq 2$.

$$
\partial_{R_{i^{\prime}}} \partial_{R_{i}} I_{C_{\ell}}=\hat{\delta}_{C_{\ell}} \alpha(n, \ldots, i+1) R_{i} \Lambda^{i} \alpha(i, \ldots, 3)\left(R_{2} \Lambda^{2} R_{2}\right) \Lambda_{2} R_{1} \Lambda_{1} \breve{\psi}
$$

Here we make use of (6.23) and $|\underline{k}|_{i}^{-1} \leq|\underline{k}|_{1}^{-1}$.

$\bullet i^{\prime}=3, i \geq 3$.

$$
\partial_{R_{i^{\prime}}} \partial_{R_{i}} I_{C_{\ell}}=\hat{\delta}_{C_{\ell}} \alpha(n, \ldots, i+1) R_{i} \Lambda^{i} \alpha(i, \ldots, 4) R_{3} \Lambda^{3} \alpha(3,2,1) \breve{\psi} .
$$

Suppose first that $1 \notin C^{2 \ell}$. Then we write $\alpha(3,2,1) \check{\psi}=R_{3} \Lambda_{3} R_{2} \Lambda_{2} R_{1} \Lambda_{1} \check{\psi}$ and apply (6.23) and $\left.\underline{\mid k}\right|_{i} ^{-1} \leq|\underline{k}|_{1}^{-1}$ to obtain the required bound. Now suppose that $1 \in C^{2 \ell}$. Writing $\hat{\delta}_{C_{\ell}}=\hat{\delta}_{C_{\ell}}^{\prime}\left(R_{2} \hat{\partial}_{2} \hat{\partial}_{1}\right)$, we have

$$
\partial_{R_{i^{\prime}}} \partial_{R_{i}} I_{C_{\ell}}=\hat{\delta}_{C_{\ell}}^{\prime} \alpha(n, \ldots, i+1) R_{i} \Lambda^{i} \alpha(i, \ldots, 4) R_{3} \Lambda^{3} R_{3} \Lambda_{3} R_{2} \check{\psi}
$$

By (6.22) we have $R_{3} \Lambda_{3} R_{2} \breve{\psi}=O\left(\sigma^{-\delta_{\lambda_{0}}}|\underline{k}|_{2}^{-1}\right)$. Thus by $|\underline{k}|_{i}^{-1} \leq|\underline{k}|_{1}^{-1}$ we obtain the required bound. - $i^{\prime}>3, i \geq i^{\prime}$. We come back to formula (6.44):

$$
\partial_{R_{i^{\prime}}} \partial_{R_{i}} I_{C_{\ell}}=\hat{\delta}_{C_{\ell}} \alpha(n, \ldots, i+1) R_{i} \Lambda^{i} \alpha\left(i, \ldots, i^{\prime}+1\right) R_{i^{\prime}} \Lambda^{i^{\prime}} \alpha\left(i^{\prime}, \ldots, 1\right) \check{\psi} .
$$

If $2,3,4 \notin C_{\ell}$, we write $\alpha\left(i^{\prime}, \ldots, 1\right) \check{\psi}=\alpha\left(i^{\prime}, \ldots, 4\right) R_{3} \Lambda_{3} R_{2} \Lambda_{2} R_{1} \Lambda_{1} \check{\psi}$ and apply (6.23) and $|\underline{k}|_{i}^{-1} \leq|\underline{k}|_{1}^{-1},\left.\underline{\mid k}\right|_{i^{\prime}} ^{-1} \leq|\underline{k}|_{3}^{-1}$ to obtain the required bound.

If $2 \in C_{\ell}$ (hence, automatically, $3 \notin C_{\ell}$ ) and $4 \notin C_{\ell}$ we have

$$
\partial_{R_{i^{\prime}}} \partial_{R_{i}} I_{C_{\ell}}=\hat{\delta}_{C_{\ell}}^{\prime} \alpha(1, \ldots, i+1) R_{i} \Lambda^{i} \alpha\left(i, \ldots, i^{\prime}+1\right) R_{i^{\prime}} \Lambda^{i^{\prime}} \alpha\left(i^{\prime}, \ldots, 4\right) R_{3} \Lambda_{3} R_{2} \breve{\psi} .
$$

In this case we apply (6.22), which gives $R_{3} \Lambda_{3} R_{2} \breve{\psi}=O\left(\sigma^{-\delta_{\lambda_{0}}}|\underline{k}|_{2}^{-1}\right)$ and we bound $|\underline{k}|_{i}^{-1} \leq|\underline{k}|_{1}^{-1}$, $|\underline{k}|_{i^{\prime}}^{-1} \leq|\underline{k}|_{3}^{-1}$ to obtain the required estimate.

If $2,4 \in C_{\ell}$, then, automatically, $i^{\prime}>4$ and we obtain

$$
\partial_{R_{i^{\prime}}} \partial_{R_{i}} I_{C_{\ell}}=\hat{\delta}_{C_{\ell}}^{\prime \prime} \alpha(n, \ldots, i+1) R_{i} \Lambda^{i} \alpha\left(i, \ldots, i^{\prime}+1\right) R_{i^{\prime}} \Lambda^{i^{\prime}} \alpha\left(i^{\prime}, \ldots, 5\right) R_{4} R_{2} \breve{\psi},
$$

where $\hat{\delta}_{C_{\ell}}=: \hat{\delta}_{C_{\ell}}^{\prime \prime}\left(R_{4} \hat{\partial}_{4} \hat{\partial}_{3}\right)\left(R_{2} \hat{\partial}_{2} \hat{\partial}_{1}\right)$. This expression can be handled by standard resolvent bounds and $|\underline{k}|_{i}^{-1} \leq|\underline{k}|_{1}^{-1},|\underline{k}|_{i^{\prime}}^{-1} \leq|\underline{k}|_{3}^{-1}$.

If $3 \in C_{\ell}$ (and therefore $2,4 \notin C_{\ell}$ ) we have

$$
\partial_{R_{i^{\prime}}} \partial_{R_{i}} I_{C_{\ell}}=\hat{\delta}_{C_{\ell}}^{\prime} \alpha(n, \ldots, i+1) R_{i} \Lambda^{i} \alpha\left(i, \ldots, i^{\prime}+1\right) R_{i^{\prime}} \Lambda^{i^{\prime}} \alpha\left(i^{\prime}, \ldots, 4\right) R_{3} R_{1} \Lambda_{1} \check{\psi},
$$

where $\hat{\delta}_{C_{\ell}}=: \hat{\delta}_{C_{\ell}}^{\prime}\left(R_{3} \hat{\partial}_{3} \hat{\partial}_{2}\right)$. This expression is handled making use of $R_{1} \Lambda_{1} \check{\psi}=O\left(\sigma^{-\delta_{\lambda_{0}}}\right)$, standard resolvent bounds and $\left.\underline{\mid k}\right|_{i} ^{-1} \leq|\underline{k}|_{1}^{-1},|\underline{k}|_{i^{\prime}}^{-1} \leq|\underline{k}|_{2}^{-1}$.

Case (1.2): $i \notin C^{2 \ell}, i^{\prime} \in C^{2 \ell}$. We can assume that $i^{\prime} \in C_{\ell}$ since otherwise $R_{i^{\prime}}$ does not appear in the expression and the result is zero. We set $\hat{\delta}_{C_{\ell}}=: \hat{\delta}_{C_{\ell}}^{\prime}\left(R_{i^{\prime}} \hat{\partial}_{i^{\prime}} \hat{\partial}_{i^{\prime}-1}\right)$ and write, making use of (6.40)

$$
\partial_{R_{i^{\prime}}} \partial_{R_{i}} I_{C_{\ell}}=\hat{\delta}_{C_{\ell}}^{\prime}\left(R_{i^{\prime}} \Lambda^{i^{\prime}} R_{i^{\prime}} \hat{\partial}_{i^{\prime}} \hat{\partial}_{i^{\prime}-1}\right) \alpha(n, \ldots, i+1) R_{i} \Lambda^{i} \alpha(i, \ldots, 1) \check{\psi}
$$

By our assumptions, $i>i^{\prime}$. We study several sub-cases:

- $i>i^{\prime}=2$.

$$
\partial_{R_{i^{\prime}}} \partial_{R_{i}} I_{C_{\ell}}=\hat{\delta}_{C_{\ell}}^{\prime} \alpha(n, \ldots, i+1) R_{i} \Lambda^{i} \alpha(i, \ldots, 3) R_{2} \Lambda^{2} R_{2} \check{\psi}
$$


In this case we apply (6.22) which gives $R_{2} \Lambda^{2} R_{2} \breve{\psi}=O\left(\sigma^{-\delta_{\lambda_{0}}}|\underline{k}|_{2}^{-1}\right)$ and estimate $|\underline{k}|_{i}^{-1} \leq|\underline{k}|_{1}^{-1}$ to obtain the required bound.

$\bullet i>i^{\prime}>2$ and $2 \notin C_{\ell}$.

$$
\partial_{R_{i^{\prime}}} \partial_{R_{i}} I_{C_{\ell}}=\hat{\delta}_{C_{\ell}}^{\prime} \alpha(n, \ldots, i+1) R_{i} \Lambda^{i} \alpha\left(i, \ldots, i^{\prime}+1\right) R_{i^{\prime}} \Lambda^{i^{\prime}} R_{i^{\prime}} \alpha\left(i^{\prime}-2, \ldots, 2\right) R_{1} \Lambda_{1} \check{\psi},
$$

where $\alpha\left(i^{\prime}-2, \ldots, 2\right)=1$ is understood for $i^{\prime}=3$. In this case we apply (6.19) which gives $R_{1} \Lambda_{1} \breve{\psi}=O\left(\sigma^{-\delta_{\lambda_{0}}}\right)$ and $|\underline{k}|_{i}^{-1} \leq|\underline{k}|_{1}^{-1},|\underline{k}|_{i^{\prime}}^{-1} \leq|\underline{k}|_{i^{\prime}-1}^{-1}$ to obtain the required bound.

$\bullet i>i^{\prime}>2$ and $2 \in C_{\ell}$ (hence $i^{\prime}>3$ ).

$$
\partial_{R_{i^{\prime}}} \partial_{R_{i}} I_{C_{\ell}}=\hat{\delta}_{C_{\ell}}^{\prime \prime} \alpha(n, \ldots, i+1) R_{i} \Lambda^{i} \alpha\left(i, \ldots, i^{\prime}+1\right) R_{i^{\prime}} \Lambda^{i^{\prime}} R_{i^{\prime}} \alpha\left(i^{\prime}-2, \ldots, 3\right) R_{2} \breve{\psi},
$$

where $\alpha\left(i^{\prime}-2, \ldots, 3\right)=1$ is understood for $i^{\prime}=4$. This case is handled by standard resolvent bounds and $|\underline{k}|_{i}^{-1} \leq|\underline{k}|_{1}^{-1},|\underline{k}|_{i^{\prime}}^{-1} \leq|\underline{k}|_{i^{\prime}-1}^{-1}$.

Case (2.1): $i \in C^{2 \ell}, i^{\prime} \notin C^{2 \ell}$ and $i>i^{\prime}$. As in the previous case, we can assume that $i \in C_{\ell}$. We set $\hat{\delta}_{C_{\ell}}=\hat{\delta}_{C_{\ell}}^{\prime}\left(R_{i} \hat{\partial}_{i} \hat{\partial}_{i-1}\right)$ and write, making use of (6.40)

$$
\begin{aligned}
\partial_{R_{i^{\prime}}} \partial_{R_{i}} I_{C_{\ell}} & =\hat{\delta}_{C_{\ell}}^{\prime}\left(R_{i} \Lambda^{i} R_{i} \hat{\partial}_{i} \hat{\partial}_{i-1}\right) \alpha\left(n, \ldots, i^{\prime}+1\right) R_{i^{\prime}} \Lambda^{i^{\prime}} \alpha\left(i^{\prime}, \ldots, 1\right) \check{\psi} \\
& =\hat{\delta}_{C_{\ell}}^{\prime} \alpha(n, \ldots, i+1) R_{i} \Lambda^{i} R_{i} \alpha\left(i-2, \ldots, i^{\prime}+1\right) R_{i^{\prime}} \Lambda^{i^{\prime}} \alpha\left(i^{\prime}, \ldots, 1\right) \check{\psi},
\end{aligned}
$$

where $\alpha\left(i-2, \ldots, i^{\prime}+1\right)=1$ for $i-2<i^{\prime}+1$. We study two sub-cases:

- $2 \notin C_{\ell}$.

$$
\partial_{R_{i^{\prime}}} \partial_{R_{i}} I_{C_{\ell}}=\hat{\delta}_{C_{\ell}}^{\prime} \alpha(n, \ldots, i+1) R_{i} \Lambda^{i} R_{i} \alpha\left(i-2, \ldots, i^{\prime}+1\right) R_{i^{\prime}} \Lambda^{i^{\prime}} \alpha\left(i^{\prime}, \ldots, 2\right) R_{1} \Lambda_{1} \check{\psi} .
$$

This is handled using $R_{1} \Lambda_{1} \breve{\psi}=O\left(\sigma^{-\delta_{\lambda_{0}}}\right)$ and $|\underline{k}|_{i}^{-1} \leq|\underline{k}|_{i-1}^{-1},\left.\underline{\mid k}\right|_{i^{\prime}} ^{-1} \leq|\underline{k}|_{1}^{-1}$.

- $2 \in C_{\ell}$.

$$
\partial_{R_{i^{\prime}}} \partial_{R_{i}} I_{C_{\ell}}=\hat{\delta}_{C_{\ell}}^{\prime \prime} \alpha(n, \ldots, i+1) R_{i} \Lambda^{i} R_{i} \alpha\left(i-2, \ldots, i^{\prime}+1\right) R_{i^{\prime}} \Lambda^{i^{\prime}} \alpha\left(i^{\prime}, \ldots, 3\right) R_{2} \check{\psi} .
$$

Here we use again $|\underline{k}|_{i}^{-1} \leq|\underline{k}|_{i-1}^{-1},|\underline{k}|_{i^{\prime}}^{-1} \leq|\underline{k}|_{1}^{-1}$.

Case (2.2): $i, i^{\prime} \in \bar{C}^{2 \ell}$. As before, we can actually assume that $i, i^{\prime} \in C_{\ell}$. We study several subcases:

- $i=i^{\prime}, i=2^{4}$.

$$
\begin{aligned}
\partial_{R_{i^{\prime}}} \partial_{R_{i}} I_{C_{\ell}} & =\hat{\delta}_{C_{\ell}}^{\prime}\left(2 R_{i} \Lambda^{i} R_{i} \Lambda^{i} R_{i} \hat{\partial}_{i} \hat{\partial}_{i-1}\right) \alpha(n, \ldots, 1) \check{\psi} \\
& =\hat{\delta}_{C_{\ell}}^{\prime} \alpha(n, \ldots, 3) 2 R_{2} \Lambda^{2} R_{2} \Lambda^{2} R_{2} \breve{\psi} .
\end{aligned}
$$

This is estimated using (6.22) and $|\underline{k}|_{2}^{-1} \leq|\underline{k}|_{1}^{-1}$.

$\bullet i=i^{\prime}, i>2,2 \notin C_{\ell}$.

$$
\partial_{R_{i^{\prime}}} \partial_{R_{i}} I_{C_{\ell}}=\hat{\delta}_{C_{\ell}}^{\prime}\left(2 R_{i} \Lambda^{i} R_{i} \Lambda^{i} R_{i} \hat{\partial}_{i} \hat{\partial}_{i-1}\right) \alpha(n, \ldots, 2) R_{1} \Lambda_{1} \check{\psi}
$$

This is estimated using $R_{1} \Lambda_{1} \breve{\psi}=O\left(\sigma^{-\delta_{\lambda_{0}}}\right),|\underline{\mid}|_{i}^{-1} \leq|\underline{k}|_{i-1}^{-1},|\underline{k}|_{i}^{-1} \leq|\underline{k}|_{1}^{-1}$.

$\bullet i=i^{\prime}, i>2,2 \in C_{\ell}$.

$$
\partial_{R_{i^{\prime}}} \partial_{R_{i}} I_{C_{\ell}}=\hat{\delta}_{C_{\ell}}^{\prime \prime}\left(2 R_{i} \Lambda^{i} R_{i} \Lambda^{i} R_{i} \hat{\partial}_{i} \hat{\partial}_{i-1}\right) \alpha(n, \ldots, 3) R_{2} \breve{\psi}
$$

\footnotetext{
${ }^{4}$ Expression (6.61) is somewhat schematic. $2 R_{i} \Lambda^{i} R_{i} \Lambda^{i} R_{i}$ stands for $R_{i}\left(\Lambda^{i}\right)^{j} R_{i}\left(\Lambda^{i}\right)^{j^{\prime}} R_{i}+\left\{j \leftrightarrow j^{\prime}\right\}$, but the indices $j, j^{\prime}=1,2,3$, coming from $\partial_{P^{j}} \partial_{P^{j^{\prime}}}$ are suppressed in our notation.
} 
Here we use again $|\underline{k}|_{i}^{-1} \leq|\underline{k}|_{i-1}^{-1},|\underline{k}|_{i}^{-1} \leq|\underline{k}|_{1}^{-1}$.

- $i>i^{\prime}$.

$$
\partial_{R_{i^{\prime}}} \partial_{R_{i}} I_{C_{\ell}}=\hat{\delta}_{C_{\ell}}^{\prime \prime}\left(R_{i} \Lambda^{i} R_{i} \hat{\partial}_{i} \hat{\partial}_{i-1}\right)\left(R_{i^{\prime}} \Lambda^{i^{\prime}} R_{i^{\prime}} \hat{\partial}_{i^{\prime}} \hat{\partial}_{i^{\prime}-1}\right) \alpha(n, \ldots, 1) \check{\psi}
$$

Here we use standard resolvent bounds and $|\underline{k}|_{i}^{-1} \leq|\underline{k}|_{i-1}^{-1},|\underline{k}|_{i^{\prime}}^{-1} \leq|\underline{k}|_{i^{\prime}-1}^{-1}$.

Proposition 6.5. There hold the bounds

$$
\begin{gathered}
\left\|\partial_{\Lambda_{i}} I_{C_{\ell}}\right\| \leq c^{n} \prod_{m^{\prime}=1}^{n}|\underline{k}|_{m^{\prime}}^{-1}, \\
\left\|\partial_{\Lambda_{i^{\prime}}} \partial_{\Lambda_{i}} I_{C_{\ell}}\right\| \leq\left. c^{n} \prod_{m^{\prime}=1}^{n} \underline{\mid}\right|_{m^{\prime}} ^{-1}, \\
\left\|\partial_{R_{i^{\prime}}} \partial_{\Lambda_{i}} I_{C_{\ell}}\right\| \leq c^{n} \sigma^{-\delta_{\lambda_{0}}} \prod_{m^{\prime}=1}^{n}|\underline{k}|_{m^{\prime}}^{-1}, \\
\left\|\partial_{\Lambda_{i}} \partial_{R_{i^{\prime}}} I_{C_{\ell}}\right\| \leq c^{n} \sigma^{-\delta_{\lambda_{0}}} \prod_{m^{\prime}=1}^{n}|\underline{k}|_{m^{\prime}}^{-1} .
\end{gathered}
$$

Proof. We first show (6.65). Since for $i \in C^{2 \ell}$ the factor $R_{i} \Lambda_{i}$ does not appear in $I_{C_{\ell}}$, we can assume $i \notin C^{2 \ell}$. Then

$$
\begin{aligned}
\partial_{\Lambda_{i}} I_{C_{\ell}} & =\hat{\delta}_{C_{\ell}} \partial_{\Lambda_{i}} \alpha(n, \ldots, 1) \check{\psi} \\
& =\left(\partial_{P} \Lambda_{i}\right) \hat{\delta}_{C_{\ell}} \alpha(n, \ldots, i+1) R_{i} \alpha(i-1, \ldots, 1) \check{\psi} \\
& =O\left(c^{n} \prod_{m^{\prime}=1}^{n}|\underline{k}|_{m^{\prime}}^{-1}\right),
\end{aligned}
$$

where we used (6.13) and the fact that the action of $\partial_{\Lambda_{i}}$ does not change the number of resolvents. Now we consider (6.66). As before we can assume that $i, i^{\prime} \notin C^{2 \ell}$. Let us first set $i \geq i^{\prime}$

$$
\begin{aligned}
\partial_{\Lambda_{i^{\prime}}} \partial_{\Lambda_{i}} I_{C_{\ell}} & =\hat{\delta}_{C_{\ell}} \partial_{\Lambda_{i^{\prime}}} \partial_{\Lambda_{i}} \alpha(n, \ldots, 1) \check{\psi} \\
& =\left(\partial_{P} \Lambda_{i}\right)\left(\partial_{P} \Lambda_{i^{\prime}}\right) \hat{\delta}_{C_{\ell}} \alpha(n, \ldots, i+1) R_{i} \alpha\left(i-1, \ldots, i^{\prime}+1\right) R_{i^{\prime}} \alpha\left(i^{\prime}-1, \ldots, 1\right) \check{\psi} \\
& =O\left(\left.c^{n} \prod_{m^{\prime}=1}^{n} \underline{\mid k}\right|_{m^{\prime}} ^{-1}\right),
\end{aligned}
$$

where we argued as in (6.70). For $i<i^{\prime}$ the argument is analogous. For $i=i^{\prime}$ we have

$$
\partial_{\Lambda_{i^{\prime}}} \partial_{\Lambda_{i}} I_{C_{\ell}}=\left(\partial_{P}^{2} \Lambda_{i}\right) \hat{\delta}_{C_{\ell}} \alpha(n, \ldots, i+1) R_{i} \alpha(i-1, \ldots, 1) \check{\psi}=O\left(c^{n} \sigma^{-\delta_{\lambda_{0}}} \prod_{m^{\prime}=1}^{n}|\underline{k}|_{m^{\prime}}^{-1}\right),
$$

where we argued as before and used that $\partial_{P}^{2} \Lambda_{i}=O\left(\sigma^{-\delta_{\lambda_{0}}}\right)$. 
To estimate (6.67) we can assume as before $i \notin C^{2 \ell}$, come back to formula (6.69), denote $\partial_{P} \Lambda_{i}$ by $O(1)$ and consider several cases:

Case 1: $i, i^{\prime} \notin C^{2 \ell}, i^{\prime}>i$.

$$
\begin{aligned}
\partial_{R_{i^{\prime}}} \partial_{\Lambda_{i}} I_{C_{\ell}} & =O(1) \hat{\delta}_{C_{\ell}} \partial_{R_{i^{\prime}}} \alpha(n, \ldots, i+1) R_{i} \alpha(i-1, \ldots, 1) \check{\psi} \\
& =O(1) \hat{\delta}_{C_{\ell}} \alpha\left(n, \ldots, i^{\prime}+1\right) R_{i^{\prime}} \Lambda^{i^{\prime}} \alpha\left(i^{\prime}, \ldots, i+1\right) R_{i} \alpha(i-1, \ldots, 1) \check{\psi}
\end{aligned}
$$

We consider several sub-cases.

- $1 \notin C^{2 \ell}, i \neq 1$.

$$
\begin{aligned}
\partial_{R_{i^{\prime}}} \partial_{\Lambda_{i}} I_{C_{\ell}} & =O(1) \hat{\delta}_{C_{\ell}} \alpha\left(n, \ldots, i^{\prime}+1\right) R_{i^{\prime}} \Lambda^{i^{\prime}} \alpha\left(i^{\prime}, \ldots, i+1\right) R_{i} \alpha(i-1, \ldots, 2) R_{1} \Lambda_{1} \breve{\psi} \\
& =O\left(c^{n} \sigma^{-\delta_{\lambda_{0}}} \prod_{m^{\prime}=1}^{n}|\underline{k}|_{m^{\prime}}^{-1}\right) .
\end{aligned}
$$

Here we used standard resolvent bounds, $R_{1} \Lambda_{1} \breve{\psi}=O\left(\sigma^{-\delta_{\lambda_{0}}}\right)$ and $|\underline{k}|_{i^{\prime}}^{-1} \leq|\underline{k}|_{1}^{-1}$.

- $1 \notin C^{2 \ell}, i=1$.

$$
\partial_{R_{i^{\prime}}} \partial_{\Lambda_{i}} I_{C_{\ell}}=O(1) \hat{\delta}_{C_{\ell}} \alpha\left(n, \ldots, i^{\prime}+1\right) R_{i^{\prime}} \Lambda^{i^{\prime}} \alpha\left(i^{\prime}, \ldots, 2\right) R_{1} \breve{\psi}
$$

First, suppose that $i^{\prime}>2$. Then (6.75) gives

$$
\partial_{R_{i^{\prime}}} \partial_{\Lambda_{i}} I_{C_{\ell}}=O(1) \hat{\delta}_{C_{\ell}} \alpha\left(n, \ldots, i^{\prime}+1\right) R_{i^{\prime}} \Lambda^{i^{\prime}} \alpha\left(i^{\prime}, \ldots, 3\right) R_{2} \Lambda_{2} R_{1} \breve{\psi}
$$

and we obtain from (6.22) and $|\underline{k}|_{i^{\prime}}^{-1} \leq|\underline{k}|_{2}^{-1}$ the required bound.

Next, suppose $i^{\prime}=2$. Then (6.75) gives

$$
\partial_{R_{i^{\prime}}} \partial_{\Lambda_{i}} I_{C_{\ell}}=O(1) \hat{\delta}_{C_{\ell}} \alpha(n, \ldots, 3) R_{2} \Lambda^{2} R_{2} \Lambda_{2} R_{1} \check{\psi}
$$

and we obtain from (6.22) the required bound.

- $1 \in C^{2 \ell}$ (and therefore $i>2$ ).

$$
\partial_{R_{i^{\prime}}} \partial_{\Lambda_{i}} I_{C_{\ell}}=O(1) \hat{\delta}_{C_{\ell}}^{\prime} \alpha\left(n, \ldots, i^{\prime}+1\right) R_{i^{\prime}} \Lambda^{i^{\prime}} \alpha\left(i^{\prime}, \ldots, i+1\right) R_{i} \alpha(i-1, \ldots, 3) R_{2} \check{\psi}
$$

This is estimated using standard resolvent bounds and $|\underline{k}|_{i^{\prime}}^{-1} \leq|\underline{k}|_{1}^{-1}$.

Case 2: $i, i^{\prime} \notin C^{2 \ell}, i^{\prime}=i$.

$$
\partial_{R_{i}} \partial_{\Lambda_{i}} I_{C_{\ell}}=O(1) \hat{\delta}_{C_{\ell}} \alpha(n, \ldots, i+1) R_{i} \Lambda^{i} R_{i} \alpha(i-1, \ldots, 1) \breve{\psi}
$$

We consider several sub-cases:

- $1 \notin C^{2 \ell}, i \neq 1$.

$$
\partial_{R_{i}} \partial_{\Lambda_{i}} I_{C_{\ell}}=O(1) \hat{\delta}_{C_{\ell}} \alpha(n, \ldots, i+1) R_{i} \Lambda^{i} R_{i} \alpha(i-1, \ldots, 2) R_{1} \Lambda_{1} \breve{\psi}
$$

Here we use standard resolvent bounds, $R_{1} \Lambda_{1} \breve{\psi}=O\left(\sigma^{-\delta_{\lambda_{0}}}\right)$ and $|\underline{k}|_{i}^{-1} \leq|\underline{k}|_{1}^{-1}$.

- $1 \notin C^{2 \ell}, i=1$.

$$
\partial_{R_{i}} \partial_{\Lambda_{i}} I_{C_{\ell}}=O(1) \hat{\delta}_{C_{\ell}} \alpha(n, \ldots, 2) R_{1} \Lambda^{1} R_{1} \check{\psi}
$$


This is estimated using (6.22).

- $1 \in C^{2 \ell}$ (and therefore $i>2$ ).

$$
\partial_{R_{i}} \partial_{\Lambda_{i}} I_{C_{\ell}}=O(1) \hat{\delta}_{C_{\ell}}^{\prime} \alpha(n, \ldots, i+1) R_{i} \Lambda^{i} R_{i} \alpha(i-1, \ldots, 3) R_{2} \breve{\psi} .
$$

where $\alpha(i-1, \ldots, 3)$ is understood to be 1 for $i=3$. This is estimated using standard resolvent bounds and $|\underline{k}|_{i}^{-1} \leq|\underline{k}|_{1}^{-1}$.

Case 3: $i, i^{\prime} \notin C^{2 \ell}, i>i^{\prime}$.

$$
\partial_{R_{i^{\prime}}} \partial_{\Lambda_{i}} I_{C_{\ell}}=O(1) \hat{\delta}_{C_{\ell}} \alpha(n, \ldots, i+1) R_{i} \alpha\left(i-1, \ldots, i^{\prime}+1\right) R_{i^{\prime}} \Lambda^{i^{\prime}} \alpha\left(i^{\prime}, \ldots, 1\right) \check{\psi}
$$

We consider several sub-cases.

- $1 \notin C^{2 \ell}, i^{\prime} \neq 1$.

$$
\partial_{R_{i^{\prime}}} \partial_{\Lambda_{i}} I_{C_{\ell}}=O(1) \hat{\delta}_{C_{\ell}} \alpha(n, \ldots, i+1) R_{i} \alpha\left(i-1, \ldots, i^{\prime}+1\right) R_{i^{\prime}} \Lambda^{i^{\prime}} \alpha\left(i^{\prime}, \ldots, 2\right) R_{1} \Lambda_{1} \check{\psi}
$$

Here we use standard resolvent bounds, $R_{1} \Lambda_{1} \breve{\psi}=O\left(\sigma^{-\delta_{\lambda_{0}}}\right)$ and $|\underline{k}|_{i^{\prime}}^{-1} \leq|\underline{k}|_{1}^{-1}$.

- $1 \notin C^{2 \ell}, i^{\prime}=1$.

$$
\partial_{R_{i^{\prime}}} \partial_{\Lambda_{i}} I_{C_{\ell}}=O(1) \hat{\delta}_{C_{\ell}} \alpha(n, \ldots, i+1) R_{i} \alpha(i-1, \ldots, 2) R_{1} \Lambda^{1} R_{1} \Lambda_{1} \check{\psi}
$$

Here we use standard resolvent bounds and $R_{1} \Lambda_{1} \check{\psi}=O\left(\sigma^{-\delta_{\lambda_{0}}}\right)$.

- $1 \in C^{2 \ell}$ (and therefore $i^{\prime}>2$ ).

$$
\partial_{R_{i^{\prime}}} \partial_{\Lambda_{i}} I_{C_{\ell}}=O(1) \hat{\delta}_{C_{\ell}}^{\prime} \alpha(n, \ldots, i+1) R_{i} \alpha\left(i-1, \ldots, i^{\prime}+1\right) R_{i^{\prime}} \Lambda^{i^{\prime}} \alpha\left(i^{\prime}, \ldots, 3\right) R_{2} \check{\psi} .
$$

This is estimated using standard resolvent bounds and $|\underline{k}|_{i^{\prime}}^{-1} \leq|\underline{k}|_{1}^{-1}$.

Case 4: $i \notin C^{2 \ell}, i^{\prime} \in C^{2 \ell}$. (Clearly we can assume that $i^{\prime} \in C_{\ell}$ ).

$$
\partial_{R_{i^{\prime}}} \partial_{\Lambda_{i}} I_{C_{\ell}}=O(1) \hat{\delta}_{C_{\ell}}^{\prime}\left(R_{i^{\prime}} \Lambda^{i^{\prime}} R_{i^{\prime}} \hat{\partial}_{i^{\prime}} \hat{\partial}_{i^{\prime}-1}\right) \alpha(n, \ldots, i+1) R_{i} \alpha(i-1, \ldots, 1) \check{\psi} .
$$

This is estimated using standard resolvent bounds and $|\underline{k}|_{i^{\prime}}^{-1} \leq|\underline{k}|_{i^{\prime}-1}^{-1}$.

Finally, we prove (6.68). In contrast to the proof of (6.67) we have to consider the case $i \in C^{2 \ell}$. (In fact, for $i=i^{\prime}$ the action of $\partial_{R_{i}}$ generates $\Lambda^{i}$ also for $\left.i \in C_{\ell}\right)$.

Case 1': $i, i^{\prime} \notin C^{2 \ell} i \neq i^{\prime}$. In this case $\partial_{R_{i}} \partial_{\Lambda_{i^{\prime}}}=\partial_{\Lambda_{i^{\prime}}} \partial_{R_{i}}$ so the respective expressions are the same as in Case 1 and Case 3 above.

Case 2': $i, i^{\prime} \notin C^{2 \ell}, i=i^{\prime}$. This situation differs from Case 2 above. In fact, since $\partial_{\Lambda_{i}}$ acts both on $\Lambda_{i}$ and $\Lambda^{i}$, we have

$$
\begin{aligned}
\partial_{\Lambda_{i}} \partial_{R_{i}} I_{C_{\ell}}= & \hat{\delta}_{C_{\ell}} \partial_{\Lambda_{i}} \alpha(n, \ldots, i+1) R_{i} \Lambda^{i} R_{i} \Lambda_{i} \alpha(i-1, \ldots, 1) \check{\psi} \\
= & O(1) \hat{\delta}_{C_{\ell}} \alpha(n, \ldots, i+1) R_{i} R_{i} \Lambda_{i} \alpha(i-1, \ldots, 1) \check{\psi} \\
& +O(1) \hat{\delta}_{C_{\ell}} \alpha(n, \ldots, i+1) R_{i} \Lambda^{i} R_{i} \alpha(i-1, \ldots, 1) \check{\psi}
\end{aligned}
$$

We consider several sub-cases:

- $1 \notin C^{2 \ell}, i \neq 1$.

$$
\begin{aligned}
\partial_{\Lambda_{i}} \partial_{R_{i}} I_{C_{\ell}}= & O(1) \hat{\delta}_{C_{\ell}} \alpha(n, \ldots, i+1) R_{i} R_{i} \Lambda_{i} \alpha(i-1, \ldots, 2) R_{1} \Lambda_{1} \breve{\psi} \\
& +O(1) \hat{\delta}_{C_{\ell}} \alpha(n, \ldots, i+1) R_{i} \Lambda^{i} R_{i} \alpha(i-1, \ldots, 2) R_{1} \Lambda_{1} \breve{\psi} .
\end{aligned}
$$


This is estimated using standard resolvent bounds, $R_{1} \Lambda_{1} \breve{\psi}=O\left(\sigma^{-\delta_{\lambda_{0}}}\right)$ and $|\underline{k}|_{i}^{-1} \leq|\underline{k}|_{1}^{-1}$.

- $1 \notin C^{2 \ell}, i=1$.

$$
\begin{aligned}
\partial_{\Lambda_{i}} \partial_{R_{i}} I_{C_{\ell}}= & O(1) \hat{\delta}_{C_{\ell}} \alpha(n, \ldots, 2) R_{1} R_{1} \Lambda_{1} \check{\psi} \\
& +O(1) \hat{\delta}_{C_{\ell}} \alpha(n, \ldots, 2) R_{1} \Lambda^{1} R_{1} \check{\psi}
\end{aligned}
$$

In addition to standard resolvent bounds, here (6.92) is handled using (6.19), whereas (6.93) using (6.22).

- $1 \in C^{2 \ell}$, (and therefore $i>2$ ).

$$
\begin{aligned}
\partial_{\Lambda_{i}} \partial_{R_{i}} I_{C_{\ell}}= & O(1) \hat{\delta}_{C_{\ell}}^{\prime} \alpha(n, \ldots, i+1) R_{i} R_{i} \Lambda_{i} \alpha(i-1, \ldots, 3) R_{2} \check{\psi} \\
& +O(1) \hat{\delta}_{C_{\ell}}^{\prime} \alpha(n, \ldots, i+1) R_{i} \Lambda^{i} R_{i} \alpha(i-1, \ldots, 3) R_{2} \breve{\psi}
\end{aligned}
$$

where $\alpha(i-1, \ldots, 3)$ is understood to be 1 for $i=3$. We estimate both expressions using standard resolvent bounds and $|\underline{k}|_{i}^{-1} \leq|\underline{k}|_{1}^{-1}$.

Case 3': $i \notin C^{2 \ell}, i^{\prime} \in \bar{C}^{2 \ell}$. This gives the same expression as Case 4 above.

Case 4': $i, i^{\prime} \in C^{2 \ell}$. Clearly is this case we can assume that $i, i^{\prime} \in C_{\ell}$ and $i=i^{\prime}$.

$$
\partial_{\Lambda_{i}} \partial_{R_{i}} I_{C_{\ell}}=O(1) \hat{\delta}_{C_{\ell}}^{\prime}\left(R_{i} R_{i} \hat{\partial}_{i} \hat{\partial}_{i-1}\right) \alpha(n, \ldots, 1) \check{\psi} .
$$

This is estimated using $|\underline{k}|_{i}^{-1} \leq|\underline{k}|_{i-1}^{-1}$.

Proposition 6.6. There hold the bounds

$$
\begin{aligned}
& \left\|\partial_{\breve{\psi}} I_{C_{\ell}}\right\| \leq c^{n} \sigma^{-\delta_{\lambda_{0}}} \prod_{m^{\prime}=1}^{n}|\underline{k}|_{m^{\prime}}^{-1}, \\
& \left\|\partial_{\breve{\psi}}^{2} I_{C_{\ell}}\right\| \leq c^{n} \sigma^{-\delta_{\lambda_{0}}} \prod_{m^{\prime}=1}^{n}|\underline{k}|_{m^{\prime}}^{-1}, \\
& \left\|\partial_{\breve{\psi}} \partial_{R_{i}} I_{C_{\ell}}\right\| \leq c^{n} \sigma^{-\delta_{\lambda_{0}}} \prod_{m^{\prime}=1}^{n}|\underline{k}|_{m^{\prime}}^{-1}, \\
& \left\|\partial_{\breve{\psi}} \partial_{\Lambda_{i}} I_{C_{\ell}}\right\| \leq c^{n} \sigma^{-\delta_{\lambda_{0}}} \prod_{m^{\prime}=1}^{n}|\underline{k}|_{m^{\prime}}^{-1}
\end{aligned}
$$

Proof. To prove (6.97), (6.98), we write for $q=1,2$

$$
\partial_{\breve{\psi}}^{q} I_{C_{\ell}}=\hat{\delta}_{C_{\ell}} \alpha(n, \ldots, 1) \partial_{P}^{q} \breve{\psi}=O\left(c^{n} \sigma^{-\delta_{\lambda_{0}}} \prod_{m^{\prime}=1}^{n}|\underline{k}|_{m^{\prime}}^{-1}\right),
$$

where we applied standard resolvent bounds and estimates (2.7).

Now we proceed to (6.99) following the lines of the proof of (6.38).

Case 1: $i \notin C^{2 \ell}$.

$$
\begin{aligned}
\partial_{\breve{\psi}} \partial_{R_{i}} I_{C_{\ell}} & =\hat{\delta}_{C_{\ell}} \partial_{R_{i}} \alpha(n, \ldots, 1) \partial_{P} \breve{\psi} \\
& =\hat{\delta}_{C_{\ell}} \alpha(n, \ldots, i+1) R_{i} \Lambda^{i} \alpha(i, \ldots, 1) \partial_{P} \breve{\psi}
\end{aligned}
$$


We consider several sub-cases.

$\bullet 1,2 \notin C^{2 \ell}, i \geq 2$.

$$
\begin{aligned}
\partial_{\breve{\psi}} \partial_{R_{i}} I_{C_{\ell}} & =\hat{\delta}_{C_{\ell}} \alpha(n, \ldots, i+1) R_{i} \Lambda^{i} \alpha(i, \ldots, 2) R_{1} \Lambda_{1} \partial_{P} \check{\psi} \\
& =\hat{\delta}_{C_{\ell}} \alpha(n, \ldots, i+1) R_{i} \Lambda^{i} \alpha(i, \ldots, 3) R_{2} \Lambda_{2} R_{1} \Lambda_{1} R \Lambda \check{\psi} \\
& =O\left(c^{n} \sigma^{-\delta_{\lambda_{0}}} \prod_{m^{\prime}=1}^{n}|\underline{\mid}|_{m^{\prime}}^{-1}\right),
\end{aligned}
$$

where we made use of $\partial_{P} \check{\psi}=R \Lambda \check{\psi},(6.24)$ and $|\underline{k}|_{i}^{-1} \leq|\underline{k}|_{2}^{-1}$ and $\alpha(i, \ldots, 3)=1$ is understood for $i=2$.

$\bullet 1,2 \notin C^{2 \ell}, i=1$.

$$
\begin{aligned}
\partial_{\breve{\psi}} \partial_{R_{i}} I_{C_{\ell}} & =\hat{\delta}_{C_{\ell}} \alpha(n, \ldots, 2) R_{1} \Lambda^{1} R_{1} \Lambda_{1} \partial_{P} \check{\psi} \\
& =\hat{\delta}_{C_{\ell}} \alpha(n, \ldots, 2) R_{1} \Lambda^{1} R_{1} \Lambda_{1} R \Lambda \breve{\psi}
\end{aligned}
$$

This is estimated using (6.24).

$\bullet 1,2 \in C^{2 \ell}$ (and therefore $i>2$ ).

$$
\partial_{\breve{\psi}} \partial_{R_{i}} I_{C_{\ell}}=\hat{\delta}_{C_{\ell}}^{\prime} \alpha(n, \ldots, i+1) R_{i} \Lambda^{i} \alpha(i, \ldots, 3) R_{2} \partial_{P} \breve{\psi} .
$$

This is estimated using standard resolvent bounds, $\partial_{P} \breve{\psi}=O\left(\sigma^{-\delta_{\lambda_{0}}}\right)$ and $|\underline{k}|_{i}^{-1} \leq|\underline{k}|_{1}^{-1}$.

$\bullet 1 \notin C^{2 \ell}, 2 \in C^{2 \ell}, i>3$.

$$
\partial_{\breve{\psi}} \partial_{R_{i}} I_{C_{\ell}}=\hat{\delta}_{C_{\ell}}^{\prime} \alpha(n, \ldots, i+1) R_{i} \Lambda^{i} \alpha(i, \ldots, 4) R_{3} R_{1} \Lambda_{1} \partial_{P} \check{\psi} .
$$

This is estimated using standard resolvent bounds, $\partial_{P} \check{\psi}=O\left(\sigma^{-\delta_{\lambda_{0}}}\right)$ and $|\underline{k}|_{i}^{-1} \leq|\underline{k}|_{2}^{-1}$.

- $1 \notin C^{2 \ell}, 2 \in C^{2 \ell}, i=1$.

$$
\partial_{\breve{\psi}} \partial_{R_{i}} I_{C_{\ell}}=\hat{\delta}_{C_{\ell}}^{\prime} \alpha(n, \ldots, 4) R_{3} R_{1} \Lambda^{1} R_{1} \Lambda_{1} R \Lambda \check{\psi} .
$$

This is estimated using (6.24) and $1 \leq|\underline{k}|_{2}^{-1}$.

Case 2: $i \in C^{2 \ell}$ (thus we can assume $i \in C_{\ell}$ ).

$$
\partial_{\breve{\psi}} \partial_{R_{i}} I_{C_{\ell}}=\hat{\delta}_{C_{\ell}}^{\prime}\left(R_{i} \Lambda^{i} R_{i} \hat{\partial}_{i} \hat{\partial}_{i-1}\right) \alpha(n, \ldots, 1) \partial_{P} \check{\psi} .
$$

Here we use $\partial_{P} \check{\psi}=O\left(\sigma^{-\delta_{\lambda_{0}}}\right)$ and $|\underline{k}|_{i}^{-1} \leq|\underline{k}|_{i-1}^{-1}$.

Finally we prove (6.100). As in the proof of (6.65) we can assume that $i \notin C^{2 \ell}$. We have

$$
\begin{aligned}
\partial_{\breve{\psi}} \partial_{\Lambda_{i}} I_{C_{\ell}} & =\hat{\delta}_{C_{\ell}} \partial_{\Lambda_{i}} \alpha(n, \ldots, 1) \partial_{P} \check{\psi} \\
& =O(1) \hat{\delta}_{C_{\ell}} \alpha(n, \ldots, i+1) R_{i} \alpha(i-1, \ldots, 1) \partial_{P} \check{\psi} \\
& =O\left(c^{n} \prod_{m^{\prime}=1}^{n}|\underline{k}|_{m^{\prime}}^{-1}\right),
\end{aligned}
$$

where we made use of $\partial_{P} \check{\psi}=O\left(\sigma^{-\delta_{\lambda_{0}}}\right)$ and standard resolvent bounds. 


\section{A Proof of estimate (2.11), (2.12) with $|\beta|=1$ and (2.13)}

In this appendix we establish estimates on $\partial_{k_{l}^{j}} f_{P, \sigma}^{n}, \partial_{k_{l}^{j}} \partial_{k_{l}^{j^{\prime}}} f_{P, \sigma}^{n}, \partial_{P^{j}} f_{P, \sigma}^{n}$ and $\partial_{k_{l}^{j}} \partial_{P^{j^{\prime}}} f_{P, \sigma}^{n}$. Since derivatives w.r.t. $k_{l}$ do not preserve the symmetry of $f_{P, \sigma}^{n}$ under permutations of photon variables, we will make the dependence on the permutation $\pi \in S_{n}$ explicit in our notation. In particular, for a given $\pi$, we will define

$$
\underline{k}_{\pi, i},\left.\quad \underline{k}\right|_{\pi, i}, \quad r(\underline{k})_{\pi, i}, \quad R_{\pi, i}, \quad R_{\pi}^{i}, \quad \Lambda_{\pi, i}, \quad \Lambda_{\pi}^{i}
$$

by replacing $k_{i^{\prime}}$ with $k_{\pi\left(i^{\prime}\right)}$ in the corresponding expressions from Subsection 6.1. The properties established in Subsection 6.1 hold, after obvious modifications, for the new objects (A.1).

\section{A.1 Proof of estimate (2.11)}

Proof of this estimate is elementary, in particular it does not require the sophisticated bounds from Theorem 6.2 but only standard resolvent estimates from Lemma 6.1. We include it here for completeness.

We recall a formula from [Fr73] stated already in (4.41) above,

$$
f_{P, \sigma}^{n}\left(k_{1}, \ldots, k_{n}\right)=\frac{1}{\sqrt{n !}} \sum_{\pi \in S_{n}}(-1)^{n}\left\langle\Omega, \prod_{i=n}^{1} \frac{1}{H_{P-\underline{k}_{\pi, i}, \sigma}-E_{P, \sigma}+|\underline{k}|_{\pi, i}} v_{\bar{\alpha}}^{\sigma}\left(k_{\pi(i)}\right) \check{\psi}_{P, \sigma}\right\rangle,
$$

where $S_{n}$ is the set of permutations of an $n$-element set and for any $\pi \in S_{n}$ we write $\underline{k}_{\pi, i}:=\sum_{i^{\prime}=1}^{i} k_{\pi\left(i^{\prime}\right)}$ and $|\underline{k}|_{\pi, i}:=\sum_{i^{\prime}=1}^{i}\left|k_{\pi\left(i^{\prime}\right)}\right|$. We define

$$
R_{\pi, i}:=\frac{1}{H_{P-\underline{k}_{\pi, i}, \sigma}-E_{P, \sigma}+|\underline{k}|_{\pi, i}}, \quad I_{\pi}:=\left(v_{\bar{\alpha}}^{\sigma}\left(k_{1}\right) \ldots v_{\bar{\alpha}}^{\sigma}\left(k_{n}\right)\right)\left\langle\Omega, \prod_{i=n}^{1} R_{\pi, i} \check{\psi}_{P, \sigma}\right\rangle,
$$

and keep in mind that both $I_{\pi}$ and $R_{\pi, i}$ depend on $k_{1}, \ldots, k_{n}$. The first derivative of $I_{\pi}$ w.r.t. $k_{l}$ has the form

$$
\begin{aligned}
\partial_{k_{l}^{j}} I_{\pi}= & \left(v_{\bar{\alpha}}^{\sigma}\left(k_{1}\right) \ldots \partial_{k_{l}^{j}} v_{\bar{\alpha}}^{\sigma}\left(k_{l}\right) \ldots v_{\bar{\alpha}}^{\sigma}\left(k_{n}\right)\right)\left\langle\Omega, \prod_{i=n}^{1} R_{\pi, i} \check{\psi}_{P, \sigma}\right\rangle \\
& +\left(v_{\bar{\alpha}}^{\sigma}\left(k_{1}\right) \ldots v_{\bar{\alpha}}^{\sigma}\left(k_{n}\right)\right) \partial_{k_{l}^{j}}\left\langle\Omega, \prod_{i=n}^{1} R_{\pi, i} \check{\psi}_{P, \sigma}\right\rangle .
\end{aligned}
$$

The second derivative of $I_{\pi}$ is given by

$$
\begin{aligned}
\partial_{k_{l}^{j}} \partial_{k_{l}^{j^{\prime}}} I_{\pi}= & \left(v_{\bar{\alpha}}^{\sigma}\left(k_{1}\right) \ldots \partial_{k_{l}^{j}} \partial_{k_{l}^{j^{\prime}}} v_{\bar{\alpha}}^{\sigma}\left(k_{l}\right) \ldots v_{\bar{\alpha}}^{\sigma}\left(k_{n}\right)\right)\left\langle\Omega, \prod_{i=n}^{1} R_{\pi, i} \check{\psi}_{P, \sigma}\right\rangle \\
& +\left(\left(v_{\bar{\alpha}}^{\sigma}\left(k_{1}\right) \ldots \partial_{k_{l}^{j}} v_{\bar{\alpha}}^{\sigma}\left(k_{l}\right) \ldots v_{\bar{\alpha}}^{\sigma}\left(k_{n}\right)\right) \partial_{k_{l}^{j^{\prime}}}\left\langle\Omega, \prod_{i=n}^{1} R_{\pi, i} \check{\psi}_{P, \sigma}\right\rangle+\left\{j \leftrightarrow j^{\prime}\right\}\right) \\
& +\left(v_{\bar{\alpha}}^{\sigma}\left(k_{1}\right) \ldots v_{\bar{\alpha}}^{\sigma}\left(k_{n}\right)\right) \partial_{k_{l}^{j}} \partial_{k_{l}^{j^{\prime}}}\left\langle\Omega, \prod_{i=n}^{1} R_{\pi, i} \check{\psi}_{P, \sigma}\right\rangle .
\end{aligned}
$$


Since for $k_{l} \in \mathcal{A}_{\sigma, k}$ (see the definition in (2.9)) we have $\left.\left|\partial_{k_{l}^{i}} \chi_{[\sigma, k)}\left(k_{l}\right)\right| \leq c \chi_{\left[\sigma, \kappa_{*}\right.}\right)\left(k_{l}\right)$ and also $\left|\partial_{k_{l}^{\prime}} \partial_{k_{l}^{\prime}} \chi_{[\sigma, k)}\left(k_{l}\right)\right| \leq c \chi_{\left[\sigma, K_{*}\right)}\left(k_{l}\right)$, we obtain that

$$
\begin{aligned}
& \left|\partial_{k_{l}^{\prime}} v_{\bar{\alpha}}^{\sigma}\left(k_{l}\right)\right| \leq \frac{c}{\left|k_{l}\right|} \lambda \frac{\chi_{\left[\sigma, k_{*}\right)}\left(k_{l}\right)\left|k_{l}\right|^{\bar{\alpha}}}{\left(2\left|k_{l}\right|\right)^{1 / 2}}, \\
& \left|\partial_{k_{l}^{\prime}} \partial_{k_{l}^{\prime}} v_{\bar{\alpha}}^{\sigma}\left(k_{l}\right)\right| \leq \frac{c}{\left|k_{l}\right|^{2}} \lambda \frac{\chi_{\left[\sigma, \kappa_{*}\right.}\left(k_{l}\right)\left|k_{l}\right|^{\bar{\alpha}}}{\left(2\left|k_{l}\right|\right)^{1 / 2}} .
\end{aligned}
$$

(We stress that $c$ may change its value from line to line). Denoting by $\left(\partial_{k_{l}^{j}} I_{\pi}\right)^{(1)}$ the first term on the r.h.s. of (A.4), and using (A.6), (6.9), we get

$$
\left|\left(\partial_{k_{l}^{j}} I_{\pi}\right)^{(1)}\right| \leq \frac{c^{n}}{\left|k_{l}\right|} \prod_{i=1}^{n}\left(\lambda \frac{\chi_{\left[\sigma, K_{k}\right)}\left(k_{i}\right)\left|k_{i}\right|^{\bar{\alpha}}}{\left(2\left|k_{i}\right|\right)^{1 / 2}}\right) \prod_{i=1}^{n} \frac{1}{|\underline{k}| \pi, i} .
$$

Now let $\left(\partial_{k_{l}^{\prime}} I_{\pi}\right)^{(2)}$ be the second term on the r.h.s. of (A.4). Let $\pi, i$ be s.t. $l \in\{\pi(1), \pi(2), \ldots, \pi(i)\}$. Then

$$
\partial_{k_{l}^{j}} R_{\pi, i}=R_{\pi, i}\left(\left(P-\underline{k}_{\pi, i}-P_{\mathrm{f}}\right)-k_{l} /\left|k_{l}\right|\right)^{j} R_{\pi, i} .
$$

(For $l \notin\{\pi(1), \pi(2), \ldots, \pi(i)\}$ the above derivative is zero). Consequently, making use of standard resolvent bounds (6.9), we obtain

$$
\left|v_{\bar{\alpha}}^{\sigma}\left(k_{\pi(i)}\right)\right|\left\|\partial_{k_{l}^{j}} R_{\pi, i}\right\| \leq \lambda \frac{\chi_{[\sigma, k)}\left(k_{\pi(i)}\right)\left|k_{\pi(i)}\right|^{\bar{\alpha}}}{\left(2\left|k_{\pi(i)}\right|\right)^{1 / 2}} \frac{c}{|\underline{k}|_{\pi, i}^{2}} \leq \frac{c}{\left|k_{l}\right|} \lambda \frac{\chi_{\left[\sigma, K_{k}\right)}\left(k_{\pi(i)}\right)\left|k_{\pi(i)}\right|^{\bar{\alpha}}}{\left(2\left|k_{\pi(i)}\right|\right)^{1 / 2}} \frac{1}{|\underline{\mid k}|_{\pi, i}},
$$

where in the last step we exploited the fact that $l \in\{\pi(1), \pi(2), \ldots, \pi(i)\}$ which gives $\left|k_{l}\right| \leq|\underline{k}|_{\pi, i}$. Thus we get that $\left(\partial_{k_{l}^{j}} I_{\pi}\right)^{(2)}$ also satisfies a bound of the form (A.8). Hence,

$$
\left|\left(\partial_{k_{l}^{j}} I_{\pi}\right)\right| \leq \frac{c^{n}}{\left|k_{l}\right|} \prod_{i=1}^{n}\left(\lambda \frac{\chi_{\left[\sigma, k_{*}\right)}\left(k_{i}\right)\left|k_{i}\right|^{\bar{\alpha}}}{\left(2\left|k_{i}\right|\right)^{1 / 2}}\right) \prod_{i=1}^{n} \frac{1}{|\underline{k}|_{\pi, i}} .
$$

Now making use of the second identity in (5.25), we conclude the proof of (2.11) in the case of $|\beta|=1$.

To cover the case $|\beta|=2$, we still have to estimate the second derivative of the product of resolvents, appearing in the last term of (A.5). We obtain from (A.9) that for $l \in\{\pi(1), \pi(2), \ldots, \pi(i)\}$

$$
\begin{aligned}
\partial_{k_{l}^{\prime}} \partial_{k_{l}^{\prime}} R_{\pi, i}=\{ & \left\{\partial_{k_{l}^{\prime}} R_{\pi, i}\right\}\left(\left(P-\underline{k}_{\pi, i}-P_{\mathrm{f}}\right)-k_{l} /\left|k_{l}\right|\right)^{j} R_{\pi, i} \\
& +R_{\pi, i}\left(\left(P-\underline{k}_{\pi, i}-P_{\mathrm{f}}\right)-k_{l} /\left|k_{l}\right|\right)^{j}\left\{\partial_{k_{l}^{\prime}} R_{\pi, i}\right\} \\
& +R_{\pi, i}\left(k_{l}^{j} k_{l}^{j^{\prime}}\left|k_{l}\right|^{-3}-\delta_{j, j^{\prime}}\left(1+\left|k_{l}\right|^{-1}\right)\right) R_{\pi, i} .
\end{aligned}
$$

From this expression, Lemma 6.1 and formula (A.10), we obtain that

$$
\left|v_{\bar{\alpha}}^{\sigma}\left(k_{\pi(i)}\right)\right|\left\|\partial_{k_{l}^{i_{l}^{\prime}}} \partial_{k_{l}^{j}} R_{\pi, i}\right\| \leq \frac{c}{\left|k_{l}\right|^{2}} \lambda \frac{\chi_{\left[\sigma, k_{*}\right)}\left(k_{\pi(i)}\right)\left|k_{\pi(i)}\right|^{\bar{\alpha}}}{\left(2\left|k_{\pi(i)}\right|\right)^{1 / 2}} \frac{1}{|\underline{k}|_{\pi, i}} .
$$


Now let $\pi$ and $i_{1}, i_{2}$ be s.t. $l \in\left\{\pi(1), \pi(2), \ldots, \pi\left(i_{1}\right)\right\}$ and $l \in\left\{\pi(1), \pi(2), \ldots, \pi\left(i_{2}\right)\right\}$. Then, making use of (A.10), we get

$$
\begin{aligned}
& \left|v_{\bar{\alpha}}^{\sigma}\left(k_{\pi\left(i_{1}\right)}\right) \| v_{\bar{\alpha}}^{\sigma}\left(k_{\pi\left(i_{2}\right)}\right)\right||| \partial_{k_{l}^{j^{\prime}}} R_{\pi, i_{1}}||\left|\partial_{k_{l}^{j}} R_{\pi, i_{2}}\right| \mid \\
& \quad \leq \frac{c}{\left|k_{l}\right|^{2}} \lambda \frac{\chi_{\left[\sigma, \kappa_{*}\right)}\left(k_{\pi\left(i_{1}\right)}\right)\left|k_{\pi\left(i_{1}\right)}\right|^{\bar{\alpha}}}{\left(2\left|k_{\pi\left(i_{1}\right)}\right|\right)^{1 / 2}} \frac{1}{|\underline{k}|_{\pi, i_{1}}} \lambda \frac{\chi_{\left[\sigma, \kappa_{*}\right.}\left(k_{\pi\left(i_{2}\right)}\right)\left|k_{\pi\left(i_{2}\right)}\right|^{\bar{\alpha}}}{\left(2\left|k_{\pi\left(i_{2}\right)}\right|\right)^{1 / 2}} \frac{1}{|\underline{k}|_{\pi, i_{2}}} .
\end{aligned}
$$

Now we use formulas (A.13) and (A.14) to estimate the last term on the r.h.s. of (A.5). The remaining terms in (A.5) are estimated with the help of (A.6), (A.7), (A.10) and Lemma 6.1. Altogether we get

$$
\left|\partial_{k_{l}^{j}} \partial_{k_{l}^{j^{\prime}}} I_{\pi}\right| \leq \frac{c^{n}}{\left|k_{l}\right|^{2}} \prod_{i=1}^{n}\left(\lambda \frac{\chi_{\left[\sigma, \kappa_{*}\right)}\left(k_{i}\right)\left|k_{i}\right|^{\bar{\alpha}}}{\left(2\left|k_{i}\right|\right)^{1 / 2}}\right) \prod_{i=1}^{n} \frac{1}{|\underline{k}|_{\pi, i}} .
$$

Making use of the second identity in (5.25) we complete the proof of (2.11) for $|\beta|=2$.

\section{A.2 Proof of estimate (2.12) with $|\beta|=1$}

We can rewrite formula (A.2) as follows

$$
\sqrt{n !}(-1)^{n} f_{P, \sigma}^{n}=v \sum_{\pi \in S_{n}}\left\langle\Omega, R_{\pi, n} \ldots R_{\pi, 1} \check{\psi}_{P, \sigma}\right\rangle
$$

where $v\left(k_{1}, \ldots, k_{n}\right):=v_{\bar{\alpha}}^{\sigma}\left(k_{1}\right) \ldots v_{\bar{\alpha}}^{\sigma}\left(k_{n}\right)$. Consequently, making use of (6.12), we have

$$
\begin{aligned}
\sqrt{n !}(-1)^{n} \partial_{P^{j}} f_{P, \sigma}^{n}=v I_{1, n}+v I_{2, n}, \text { where } I_{1, n} & :=\sum_{\pi \in S_{n}}\left\langle\Omega, R_{\pi, n} \ldots R_{\pi, 1} \partial_{P^{j}} \check{\psi}_{P, \sigma}\right\rangle, \\
I_{2, n} & :=\sum_{\pi \in S_{n}} \sum_{\ell=1}^{n}\left\langle\Omega, R_{\pi, n} \ldots R_{\pi, \ell} \Lambda_{\pi}^{\ell} R_{\pi, \ell} \ldots R_{\pi, 1} \check{\psi}_{P, \sigma}\right\rangle
\end{aligned}
$$

with $\Lambda_{\pi}^{\ell}:=\Lambda+\underline{k}_{\pi, \ell}$ (see the definition of $\Lambda$ in (6.4)). By estimate (2.7), Lemma 6.1 and the second identity in (5.25) we get

$$
\left|v I_{1, n}\right| \leq \frac{1}{\sigma^{\delta_{\lambda_{0}}}} g_{\sigma}^{n}
$$

which gives one contribution to estimate (2.12).

To handle $I_{2, n}$, we apply (6.11) i.e. $R_{\pi, i}:=R_{\pi}^{i}-R_{\pi, i}\left(\underline{k}_{\pi, i} \cdot \Lambda\right) R_{\pi}^{i}$, where $R_{\pi}^{i}:=\left(H_{P, \sigma}-E_{P, \sigma}+r(\underline{k})_{\pi, i}\right)^{-1}$. In the definition of $R_{\pi, i}$ the reader can recognize a truncated resolvent expansion. In the scalar product defining $I_{2, n}$ below, we use this expansion on each $R_{\pi, i}, 1 \leq i \leq \ell$, starting from $R_{\pi, 1}$. Namely, we write

$$
\begin{aligned}
I_{2, n}= & \sum_{\pi \in S_{n}} \sum_{\ell=1}^{n}\left\langle\Omega, R_{\pi, n} \ldots R_{\pi, \ell} \Lambda_{\pi}^{\ell} R_{\pi, \ell} \ldots R_{\pi, 1} \check{\psi}_{P, \sigma}\right\rangle \\
= & \sum_{\pi \in S_{n}} \sum_{\ell=1}^{n} \sum_{i=1}^{\ell}(-)\left\langle\Omega, R_{\pi, n} \ldots R_{\pi, \ell} \Lambda_{\pi}^{\ell} R_{\pi, \ell} \ldots R_{\pi, i}\left(k_{\pi, i} \cdot \Lambda\right) R_{\pi}^{i} \ldots R_{\pi}^{1} \check{\Psi}_{P, \sigma}\right\rangle \\
& +\sum_{\pi \in S_{n}} \sum_{\ell=1}^{n}\left\langle\Omega, R_{\pi, n} \ldots R_{\pi, \ell} \Lambda_{\pi}^{\ell} R_{\pi}^{\ell} \ldots R_{\pi}^{1} \check{\psi}_{P, \sigma}\right\rangle .
\end{aligned}
$$


Now making use of Lemma 6.1, the relations

$$
R_{\pi}^{i} \check{\psi}_{P, \sigma}=r(\underline{k})_{\pi, i}^{-1} \breve{\psi}_{P, \sigma}, \quad R_{\pi, i} \Lambda \check{\psi}_{P, \sigma}=O\left(\sigma^{-\delta_{\lambda_{0}}}\right), \quad R_{\pi, \ell} \Lambda_{\pi}^{\ell} \breve{\psi}_{P, \sigma}=O\left(\sigma^{-\delta_{\lambda_{0}}}\right)
$$

(cf. Corollary 6.3), the estimate $|\underline{k}|_{\pi, \ell}^{-1} \leq|\underline{k}|_{\pi, i}^{-1}$ for $\ell \geq i$, and the second identity in (5.25), we obtain

$$
\left|\nu I_{2, n}\right| \leq \frac{1}{\sigma^{\delta_{\lambda_{0}}}} g_{\sigma}^{n}
$$

which concludes the proof of estimate (2.12) with $|\beta|=1$.

\section{A.3 Proof of estimate (2.13)}

Given the results from the last two subsections it is easy to estimate the mixed derivative $\partial_{k_{l}^{j}} \partial_{P j^{\prime}} f_{P, \sigma^{*}}^{n}$. Starting from formula (A.17) we write

$$
\sqrt{n !}(-1)^{n} \partial_{k_{l}^{j}} \partial_{P^{j^{\prime}}} f_{P, \sigma}^{n}=\partial_{k_{l}^{j}}\left(v I_{1, n}\right)+\left(\partial_{k_{l}^{j}} v\right) I_{2, n}+v \partial_{k_{l}^{j}} I_{2, n} .
$$

We note that the considerations in Subsection A.1 are unchanged if $\breve{\psi}_{P, \sigma}$ is replaced with $\partial_{P^{j^{\prime}}} \breve{\psi}_{P, \sigma}$, except for an additional factor $\sigma^{-\delta_{\lambda_{0}}}$ in the estimates coming from (2.7). Thus we get immediately

$$
\left|\partial_{k_{l}^{j}}\left(v I_{1, n}\right)\right| \leq \frac{1}{\sigma^{\delta_{\lambda_{0}}}}\left|k_{l}\right|^{-1} g_{\sigma}^{n} .
$$

Next, combining the analysis of $I_{2, n}$ from Subsection A.2 with the bound (A.6) we obtain

$$
\left|\left(\partial_{k_{l}^{j}} v\right) I_{2, n}\right| \leq \frac{1}{\sigma^{\delta_{\lambda_{0}}}}\left|k_{l}\right|^{-1} g_{\sigma}^{n} .
$$

As for the last term on the r.h.s. of (A.24), by inspection of (A.20) and (A.21) we see that the action of $\partial_{k_{l}^{j}}$ on $I_{2, n}$ leads to an additional factor $\left|k_{l}\right|^{-1}$ in the estimates. In fact, the relevant ingredients are

$$
R_{\pi}^{i} \check{\psi}_{P, \sigma}=r(\underline{k})_{\pi, i}^{-1} \check{\psi}_{P, \sigma}, \quad \partial_{k_{l}^{j}} r(\underline{k})_{\pi, i}^{-1}=O\left(\left|k_{l}\right|^{-1}|\underline{k}|_{\pi, i}^{-1}\right), \quad \partial_{k_{l}^{j}} \underline{k}_{\pi, i}=O(1)=O\left(|\underline{k}|_{\pi, i} /\left|k_{l}\right|\right)
$$

and the fact that $\partial_{k_{l}^{j}} R_{\pi, i}=R_{\pi, i}\left(\left(P-\underline{k}_{\pi, i}-P_{\mathrm{f}}\right)-k_{l} /\left|k_{l}\right|\right)^{j} R_{\pi, i}=O\left(\left|k_{l}\right|^{-1}|\underline{k}|_{\pi, i}^{-1}\right)$, cf. (A.9). Using this and the second identity in (5.25) we conclude the proof.

\section{B Proof of Proposition 4.8}

Our discussion here is similar to the first part of Appendix D of [DP17]. In Subsection 4.1 we defined $\mathbb{R}^{3} \ni k \mapsto b_{W}(k) \psi$ for $\psi \in C^{\infty}\left(H_{P \text {,free }}\right)$ as vector-valued distributions. For some vectors $\psi \in C^{\infty}\left(H_{P \text {,free }}\right)$ we can also define $b(k) \psi$ pointwise in $k$ as follows: Let $\eta \in C_{0}^{\infty}\left(\mathbb{R}^{3}\right)$ be s.t. $\eta \geq 0$, $\eta(0)=1, \int \eta\left(k^{\prime}\right) d^{3} k^{\prime}=1, \eta(k)=\eta(-k)$ so that $\eta_{k}^{\varepsilon}\left(k^{\prime}\right):=\varepsilon^{-3} \eta\left(\left(k^{\prime}-k\right) / \varepsilon^{-1}\right)$ is an approximating sequence of $k^{\prime} \mapsto \delta\left(k^{\prime}-k\right)$ as $\varepsilon \rightarrow 0$. We say that $\psi \in C^{\infty}\left(H_{P \text {,free }}\right)$ is in $D\left(b_{W}(k)\right)$ if the following limit exists in norm

$$
b_{W}(k) \psi:=\lim _{\varepsilon \rightarrow 0} b_{W}\left(\eta_{k}^{\varepsilon}\right) \psi
$$


and gives an element of $C^{\infty}\left(H_{P \text {,free }}\right)$. We also require that $\lim _{\varepsilon \rightarrow 0} b_{W}\left(g \eta_{k}^{\varepsilon}\right) \psi$ exists and equals $g(k) b_{W}(k) \psi$ for any continuous function $g$. The domain of the product $D\left(b_{W}\left(k_{1}\right) \ldots b_{W}\left(k_{m}\right)\right)$ is defined iteratively. As we showed in Lemma D.1 of [DP17], relation (B.1) is consistent with the definition as a distribution.

To prove (4.51), we first recall relation (4.22)

$$
\begin{aligned}
b_{W}\left(k_{m}\right) \ldots b_{W}\left(k_{1}\right) H_{P, \sigma} \psi= & H_{P, \sigma ; k_{m}, \ldots, k_{1}} b_{W}\left(k_{m}\right) \ldots b_{W}\left(k_{1}\right) \psi \\
& +\sum_{i=1}^{m} F_{P, \sigma}\left(k_{i}, k_{1}+\cdots \check{i} \cdots+k_{m}\right) b_{W}\left(k_{m}\right) \cdots \check{i} \cdots b_{W}\left(k_{1}\right) \psi \\
& +\sum_{1 \leq i<i^{\prime} \leq m} G_{P, \sigma}\left(k_{i}, k_{i^{\prime}}\right) b_{W}\left(k_{m}\right) \cdots \check{i^{\prime}} \cdots \check{i} \cdots b_{W}\left(k_{1}\right) \psi,
\end{aligned}
$$

which holds in the sense of distributions. Now we set $\psi=\psi_{P, \sigma}$ and proceed by induction. We suppose that $\psi_{P, \sigma} \in D\left(b_{W}\left(k_{1}\right) \ldots b_{W}\left(k_{m-1}\right)\right)$ for any $k_{1}, \ldots k_{m-1} \in \mathbb{R}^{3} \backslash\{0\}$ and we will conclude from this that $\psi_{P, \sigma} \in D\left(b_{W}\left(k_{1}\right) \ldots b_{W}\left(k_{m-1}\right) b\left(k_{m}\right)\right)$. (For $m=1$ the former condition is understood to be empty and thus trivially satisfied). To this end, we set

$$
\begin{aligned}
& A\left(k_{m}^{\prime}\right):=\left(E_{P, n}-|\underline{k}|_{m-1}-\left|k_{m}^{\prime}\right|-H_{P-\underline{k}_{m-1}-k_{m}^{\prime}, n}\right), \\
& F_{m}^{i}:=F_{P, \sigma}\left(k_{i}, k_{1}+\cdots \breve{i} \cdots+k_{m}\right), \\
& B_{m-1}:=b_{W}\left(k_{m-1}\right) \ldots b_{W}\left(k_{1}\right), \\
& B_{m-1}^{i}:=b_{W}\left(k_{m-1}\right) \ldots \check{i} \ldots b_{W}\left(k_{1}\right), \\
& B_{m-1}^{i^{\prime}, i}:=b_{W}\left(k_{m-1}\right) \cdots \check{i^{\prime}} \cdots \breve{i} \cdots b_{W}\left(k_{1}\right),
\end{aligned}
$$

where we use the notation $\underline{k}_{m}:=k_{1}+\cdots+k_{m}$ and $|\underline{k}|_{m}:=\left|k_{1}\right|+\cdots+\left|k_{m}\right|$. Next, for some $k_{m} \neq 0$, we rewrite (B.2) as follows

$$
\begin{aligned}
b_{W}(f) B_{m-1} \psi_{P, \sigma}= & F_{m}^{m}(f) A\left(k_{m}\right)^{-1} B_{m-1} \psi_{P, \sigma}+A\left(k_{m}\right)^{-1} \sum_{i=1}^{m-1}\left(F_{m}^{i} b_{W}\right)(f) B_{m-1}^{i} \psi_{P, \sigma} \\
& +A\left(k_{m}\right)^{-1} \sum_{i=1}^{m-1} G\left(k_{i}, f\right) B_{m-1}^{i} \psi_{P, \sigma}+A\left(k_{m}\right)^{-1} \sum_{1 \leq i<i^{\prime} \leq m-1} G_{P, \sigma}\left(k_{i}, k_{i^{\prime}}\right) b_{W}(f) B_{m-1}^{i^{\prime}, i} \psi_{P, \sigma} \\
& +\int d^{3} k_{m}^{\prime} f\left(k_{m}^{\prime}\right) A\left(k_{m}\right)^{-1}\left(A\left(k_{m}\right)-A\left(k_{m}^{\prime}\right)\right) b_{W}\left(k_{m}^{\prime}\right) B_{m-1} \psi_{P, \sigma}
\end{aligned}
$$

where we smeared both sides of (B.2) in one variable with a test-function $f$ vanishing near zero and $F_{m}^{m}(f), G\left(k_{i}, f\right),\left(F_{m}^{i} b_{W}\right)(f)$ denote smearing of the respective expressions with $f$. Now we analyze (B.10). For simplicity of notation we set in the following $k:=k_{m}, k^{\prime}:=k_{m}^{\prime}$. First, we note that

$$
A(k)-A\left(k^{\prime}\right)=L_{k}\left(k^{\prime}\right)+\left(P^{\prime}-P_{\mathrm{f}}\right) M_{k}\left(k^{\prime}\right),
$$

where $P^{\prime}:=P-\underline{k}_{m-1}$ and we defined the functions

$$
L_{k}\left(k^{\prime}\right):=\left(\left|k^{\prime}\right|-|k|\right)-\frac{1}{2}\left(k-k^{\prime}\right)\left(k^{\prime}+k\right), \quad M_{k}\left(k^{\prime}\right):=\left(k-k^{\prime}\right) .
$$

With these definitions we can write

$$
(B .10)=A(k)^{-1} b\left(L_{k} f\right) B_{m-1} \psi_{P, \sigma}+A(k)^{-1}\left(P^{\prime}-P_{\mathrm{f}}\right) b\left(M_{k} f\right) B_{m-1} \psi_{P, \sigma} .
$$


To conclude the argument, we substitute this back to (B.8)-(B.10), set $f=g \eta_{k}^{\varepsilon}$, note that (B.8), (B.9) converge as $\varepsilon \rightarrow 0$ to the desired quantities by the inductive hypothesis and (B.13) tends to zero in this limit since $M_{k}(k)=L_{k}(k)=0$ and $\eta_{k}^{\varepsilon}\left(k^{\prime}\right) \rightarrow \delta\left(k-k^{\prime}\right)$. To show vanishing of (B.13), one combines an iteration argument with energy bounds as in the last part of the proof of Lemma D.3 of [DP17].

\section{References}

[AH12] A. Abdesselam and D. Hasler, Analyticity of the ground state energy for massless Nelson models. Commun. Math. Phys. 310, (2012) 511-536.

[DP12] W. Dybalski and A. Pizzo, Coulomb scattering in the massless Nelson model I. Foundations of two-electron scattering. J. Stat. Phys. 154, (2014) 543-587.

[DP17] W. Dybalski and A. Pizzo, Coulomb scattering in the massless Nelson model II. Regularity of ground states. Preprint arXiv:1302.5012v2.

[FFS14] J. Faupin, J. Fröhlich and B. Schubnel, Analyticity of the self-energy in total momentum of an atom coupled to the quantized radiation field J. Funct. Anal. 267, 4139-4196.

[Fr73] J. Fröhlich, On the infrared problem in a model of scalar electrons and massless, scalar bosons. Ann. Inst. H. Poincaré Sect. A (N.S.) 19, (1973) 1-103.

[Fr] J. Fröhlich, unpublished notes.

[Fr74] J. Fröhlich, Existence of dressed one electron states in a class of persistent models. Fortschr. Phys. 22, (1974) 158-198.

[FP10] J. Fröhlich and A. Pizzo, Renormalized electron mass in non-relativistic QED. Commun. Math. Phys. 294, (2010) 439-470.

[JMNP07] M.A. Jivulescu, A. Messina, A. Napoli and F. Petruccione, Exact treatment of linear difference equations with noncommutative coefficients. Mathematical Methods in the Applied Sciences 30, (2007) 2147-2153.

[JNM08] M.A. Jivulescu, A. Messina, A. Napoli, General solution of a second order nonhomogeneous linear difference equation with non-commutative coefficients. Applied Mathematics \& Information Sciences 4, (2010) 1-14.

[KM12] M. Könenberg and O. Matte, The mass-shell in the semi-relativistic Pauli-Fierz model. Ann. Henri Poincaré 15, (2014) 863-915.

[Pi03] A. Pizzo, One-particle (improper) states in Nelson's massless model. Ann. Henri Poincaré 4, (2003) 439-486.

[Pu15] G. Puhlfürst, Non-commutative recurrence relations for scattering amplitudes. PhD thesis, Ludwig-Maximilians-Universität München, 2015. 
[St17] A. Strominger, Lectures on the infrared structure of gravity and gauge theory. Preprint arXiv: 1703.05448. 